\title{
The Mass Shell in the Semi-Relativistic Pauli-Fierz Model
}

\author{
Martin Könenberg and Oliver Matte
}

\begin{abstract}
We consider the semi-relativistic Pauli-Fierz model for a single free electron interacting with the quantized radiation field. Employing a variant of Pizzo's iterative analytic perturbation theory we construct a sequence of ground state eigenprojections of infra-red cutoff, dressing transformed fiber Hamiltonians and prove its convergence, as the cutoff goes to zero. Its limit is the ground state eigenprojection of a certain renormalized fiber Hamiltonian. The ground state energy is an exactly twofold degenerate eigenvalue of the renormalized Hamiltonian, while it is not an eigenvalue of the original fiber Hamiltonian unless the total momentum is zero. These results hold true, for total momenta inside a ball about zero of arbitrary radius $\mathfrak{p}>0$, provided that the coupling constant is sufficiently small depending on $\mathfrak{p}$ and the ultra-violet cutoff. Along the way we prove twice continuous differentiability and strict convexity of the ground state energy as a function of the total momentum inside that ball.
\end{abstract}

\section{Introduction and Main Results}

\subsection{The General Framework}

The scope of this paper is a mathematically rigorous investigation of the infrared (IR) problem for a single free electron in a simplified model for quantum electrodynamics (QED). Due to the absence of external potentials the Hamiltonian for the electron interacting with the quantized radiation field is translation invariant, and it is, therefore, possible to decompose the Hamiltonian with respect to the spectrum of the total momentum operator, i.e. the generators of translations. In mathematical terms the Hamiltonian is unitarily equivalent to a direct integral of fiber Hamiltonians. The ground state energy of the fiber Hamiltonians as a function of the total momentum is called the mass shell. While the mass shell can be computed explicitly when the interaction between the electron and the photon field is set equal to zero, almost nothing is known 
a priori about its shape and regularity when the interaction with the ultraviolet cut-off quantized radiation field is turned on. The shape of the mass shell reflects the influence of the quantized radiation field onto the electron; see for instance [2], where the mass shell enters into the implementation of an effective dynamics for a non-relativistic (NR) electron coupled to the quantized radiation field in a slowly varying external potential. Another example for the importance of the mass shell is the definition of the renormalized electron mass as its inverse second derivative at zero. For this definition to make sense it is of course necessary to prove sufficient regularity and strict convexity of the mass shell. In the NR Pauli-Fierz model this has been achieved in [17]. Finally, information on the mass shell is an essential input for the construction of infra-particle scattering states which, in the one-electron sector, has recently been carried out in [12] for the NR Pauli-Fierz model and earlier in [30,31] for Nelson's model.

While a general guideline for the mathematical treatment of the IR problem and in particular of infra-particle scattering has been settled long ago in a study of the Nelson model $[14,15]$, several problems could not be treated until the past decade. The main reason for these difficulties is the occurrence of, in general, infinitely many soft photons in the ground states (understood as positive linear functionals on a certain observable algebra) of the combined electron-photon system described by a fiber Hamiltonian. Intuitively, due to the vanishing photon mass arbitrarily small contributions to the total energy may be spread over arbitrarily many low-energetic photons. This is reflected in two technical difficulties: the spectrum of the fiber Hamiltonians is continuous up to their ground state energies and their ground states (if any) may give rise to disjoint coherent IR representation spaces for a certain algebra of observables; see [33]. In fact, unless the total momentum is zero, the ground states of the fiber Hamiltonians (still understood as functionals) are not normal in the Fock representation and the fiber Hamiltonians do not possess ground state eigenvectors. The analysis of these phenomena again requires non-trivial knowledge about the mass shell: for a start one can directly show that a fiber Hamiltonian attached to a total momentum $\boldsymbol{p}$, where the mass shell is assumed to be differentiable, can only have a ground state eigenvector provided that the derivative of the mass shell at $\boldsymbol{p}$ vanishes. In the NR situation this has been done in [18]. To show, however, that a non-zero $\boldsymbol{p}$ entails a non-zero derivative one needs to prove strict convexity of the mass shell (or at least uniform convexity of approximations to it $[3,9]$ ) and to verify that its minimum is attained at zero as it is certainly expected. In the NR Pauli-Fierz model the disjointness of coherent IR representations associated with distinct total momenta has been established in [10] based on bounds on the renormalized electron mass from [9].

There are mainly two sophisticated multi-scale techniques which are responsible for the recent progress in this area. The first one is the spectral renormalization group introduced by Bach, Fröhlich, and Sigal. In the present setting it was applied first in [9] and later on in [3] to the standard model of NR QED. Here we will apply the second method, namely 
the iterative analytic perturbation theory (IAPT) developed mainly by Pizzo in his analysis of Nelson's model [30,31] and later applied in [11,17] to the translation-invariant NR Pauli-Fierz model. In [4-6] the IAPT has been used to provide expansions of atomic ground state energies and eigenvectors and of scattering amplitudes. More recently, the removal of the ultra-violet cutoff in Nelson's model has been studied by means of the IAPT [8]. A related procedure based on continuous flows instead of discrete iteration steps as in the IAPT can be found in [7]. We should mention here that in the Nelson model it is actually possible to show real analyticity of the mass shell near zero [1] thanks to the applicability of path integral and cluster expansion techniques.

In contrast to the previously mentioned works we will investigate the IR problem for the semi-relativistic (SR) Pauli-Fierz model, i.e. we start from the relativistic energy-momentum dependency for the electron and introduce the quantized radiation field as usual via minimal coupling. The study of the so-obtained square-root Hamiltonian was initiated in [28] where the bottom of the essential spectrum of fiber Hamiltonians is characterized. The main results of [28] are, however, obtained under certain simplifying assumptions that render the model more IR regular. A scalar square-root Hamiltonian appeared earlier in the mathematical analysis of Rayleigh scattering [16]. In the study of binding in the presence of electrostatic potentials the IR singularities of the SR Pauli-Fierz model can be dealt with similarly as in the NR model; see [19,22-24,27] where binding energies, exponential localization of low-energy states, and the existence of ground state eigenvectors are investigated (in contrast to the NR model the SR one with Coulomb potential becomes unstable if the Coulomb coupling constant is too large). One may hope, however, that the large energy behavior of the interaction between the electron and the photon field is described more realistically by SR operators. In fact, thanks to the SR nature of our model we may choose an upper bound on the moduli of total momenta covered by our results as large as we please, at the expense, however, of choosing the coupling constant sufficiently small. The latter restriction is likely to be a technical one, while in the NR setting the results of $[10,11,17]$ are not expected to be true for large total momenta where the influence of soft photons is dominant in the low-energy states due to the NR dispersion relation of the electron.

Fiber Hamiltonians with a relativistic kinetic energy for the matter particles and linearly coupled radiation fields appear, e.g., in [14,29] and more recently in [13], where it is shown that even a mass renormalization in the linearly coupled model cannot prevent the mass shell from becoming flat as the ultra-violet cut-off tends to infinity.

Although one might expect the analysis of square-root Hamiltonians to be technically more involved we are able to establish essentially all main results of $[10,11,17]$ in the SR case, viz. existence of the renormalized electron mass (Theorem 1.1(1) below; cf. [17] for the NR case), existence of ground state eigenvectors at total momentum zero (Theorem 1.1(2); cf. [9,11]), absence of eigenvalues at non-zero momenta (Theorem 1.1(3); cf. [10,18]), existence of 
ground state eigenvectors of renormalized Hamiltonians (Theorem 1.2(1); cf. $[9,11]$ ), and disjointness of coherent IR representations (Theorem 1.2(3); cf. [10]). Needless to say, in many details and aspects we profit from the works on the NR Pauli-Fierz operator and on Nelson's operator [30,31]. Concerning the IAPT, however, we also propose several new arguments or alterations of earlier ones within its general framework which, as we hope, will be helpful in future investigations including the NR case (To mention some keywords for the experts: we do not employ contour integrals and avoid repeated Neumann series expansions and certain bounds relating expectations of operators with expectations of their absolute values; by a minor modification of the dressing transforms we avoid the discussion of intermediate Hamiltonians). As a novel application of the IAPT we further found a very simple and entirely selfcontained discussion of the disjointness of coherent IR representations, which avoids the use of infinite tensor products and any reference to abstract results on CCR representations.

Another novelty achieved here is a proof of the exact twofold degeneracy of the ground state eigenvalues of renormalized fiber Hamiltonians in the presence of spin; see Theorem 1.2(1) below (without spin the non-degeneracy follows from Perron-Frobenius arguments [14]). As our corresponding argument is essentially based on a certain relative form bound required to get the IAPT started, it is clear that it also applies mutatis mutandis to Nelson's model (proving uniqueness of ground states) and to the NR Pauli-Fierz model (for which it was still an open problem [20] to prove exact two-fold degeneracy).

\subsection{The Model and Main Results}

In this subsection we explain the model under investigation and state our main results. The organization of this article is explained in Sect. 1.3.

The semi-relativistic (SR) Pauli-Fierz Hamiltonian, $\mathbb{H}_{\mathrm{sr}}$, generates the dynamics of a single free electron with spin one-half and mass unity interacting with the quantized radiation field. The interaction is introduced via minimal coupling, $-i \nabla_{\boldsymbol{x}} \mapsto-i \nabla_{\boldsymbol{x}}+\mathfrak{e} \mathbb{A}$, of the quantized vector potential, $\mathbb{A}$, with sharp ultra-violet cutoff at $\kappa>0$,

$$
\mathbb{A}(\boldsymbol{x}):=\sum_{\lambda \in \mathbb{Z}_{2}} \int_{|\boldsymbol{k}|<\kappa} \frac{\boldsymbol{\varepsilon}(\boldsymbol{k}, \lambda)}{(2|\boldsymbol{k}|)^{1 / 2}}\left(e^{-i \boldsymbol{k} \cdot \boldsymbol{x}} a^{\dagger}(\boldsymbol{k}, \lambda)+e^{i \boldsymbol{k} \cdot \boldsymbol{x}} a(\boldsymbol{k}, \lambda)\right) \frac{\mathrm{d}^{3} \boldsymbol{k}}{(2 \pi)^{3 / 2}} .
$$

The parameter $\mathfrak{e}>0$, chosen sufficiently small later on, models the elementary charge; in our units the square of the elementary charge equals Sommerfeld's fine-structure constant whose physical value is roughly $1 / 137$. Throughout this article $\mathbb{A}$ satisfies the Coulomb gauge condition, $\nabla_{\boldsymbol{x}} \cdot \mathbb{A}=0$. Therefore, the polarization vectors appearing in (1.1) are chosen such that $\{\boldsymbol{k} /|\boldsymbol{k}|, \boldsymbol{\varepsilon}(\boldsymbol{k}, 0)$, $\varepsilon(\boldsymbol{k}, 1)\}$ is an oriented orthonormal basis of $\mathbb{R}^{3}$. A lot of attention by mathematicians has been attracted by the standard model of non-relativistic QED where the dispersion relation for a non-interacting electron is $\boldsymbol{p}^{2} / 2$. In contrast we choose a relativistic dispersion relation for the electron, i.e. its energy at momentum $\boldsymbol{p}$ is $\left(\boldsymbol{p}^{2}+1\right)^{1 / 2}$ when the radiation field is turned off. The resulting 
total Hamiltonian, thus, is

$$
\mathbb{H}_{\mathrm{sr}}:=\sqrt{\left(\boldsymbol{\sigma} \cdot\left(-i \nabla_{\boldsymbol{x}} \otimes \mathbb{1}+\mathfrak{e} \mathbb{A}\right)\right)^{2}+\mathbb{1}}+\mathbb{1} \otimes H_{\mathrm{f}}
$$

Here $H_{\mathrm{f}}$ is the energy of the free radiation field and $\boldsymbol{\sigma}=\left(\sigma_{1}, \sigma_{2}, \sigma_{3}\right)$ denotes the vector of Pauli spin matrices. The Hilbert space for this Hamiltonian is the tensor product of the electron Hilbert space $L^{2}\left[\mathbb{R}_{\boldsymbol{x}}^{3}, \mathbb{C}^{2}\right]$ and the state space of the photon field which is the bosonic Fock space, $\mathscr{F}$, over $L^{2}\left[\mathbb{R}_{\boldsymbol{k}}^{3} \times \mathbb{Z}_{2}\right]$. Since no external potentials are present the Hamiltonian is space translation invariant and we may hence decompose it with respect to the generalized eigenspaces of the total momentum operator. In mathematical terms $\mathbb{H}_{\text {sr }}$ is unitarily equivalent to a direct integral of fiber Hamiltonians, $\mathcal{H}(\boldsymbol{p})$,

$$
\mathbb{H}_{\mathrm{sr}} \cong \int_{\mathbb{R}^{3}}^{\oplus} \mathcal{H}(\boldsymbol{p}) \mathrm{d}^{3} \boldsymbol{p} .
$$

In a sense the Hamiltonian $\mathcal{H}(\boldsymbol{p})$ describes an idealized situation where the total momentum of the system is fixed at $\boldsymbol{p} \in \mathbb{R}^{3}$. It is given by

$$
\mathcal{H}(\boldsymbol{p}):=\sqrt{\left(\boldsymbol{\sigma} \cdot\left(\boldsymbol{p}-\mathbf{p}_{\mathrm{f}}+\mathfrak{e} \boldsymbol{A}\right)\right)^{2}+\mathbb{1}}+H_{\mathrm{f}},
$$

where $\boldsymbol{A}=\mathbb{A}(\mathbf{0})$ and $\mathbf{p}_{\mathrm{f}}$ is the field momentum operator. We postpone a rigorous definition of $\mathcal{H}(\boldsymbol{p})$ to Sect. 2. Here we just mention that $\mathcal{H}(\boldsymbol{p})$ acts in the Hilbert space $\mathbb{C}^{2} \otimes \mathscr{F}$ (careful discussions of (1.2) and (1.3) can be found, e.g., in [25] and [23], respectively). One of the main objectives of this paper is the study of the mass-shell, that is, the ground state energy, $E(\boldsymbol{p})$, of $\mathcal{H}(\boldsymbol{p})$ as a function of the total momentum,

$$
E(\boldsymbol{p}):=\inf \operatorname{Spec}[\mathcal{H}(\boldsymbol{p})], \quad \boldsymbol{p} \in \mathbb{R}^{3} .
$$

In order to state our first main result we introduce the notation

$$
\mathcal{B}_{\mathfrak{p}}:=\left\{\boldsymbol{p} \in \mathbb{R}^{3}:|\boldsymbol{p}|<\mathfrak{p}\right\},
$$

for the set of all total momenta taken into account. Here the radius $0<\mathfrak{p}<\infty$ as well as the ultra-violet cutoff $0<\kappa<\infty$ can be chosen arbitrarily large. The elementary charge, $0<\mathfrak{e} \leqslant \mathfrak{e}_{0}$, is treated as a small parameter with an upper bound $\mathfrak{e}_{0}$ depending on $\mathfrak{p}$ and $\kappa$.

Theorem 1.1. For all $\kappa, \mathfrak{p}>0$, we find $\mathfrak{e}_{0}>0$ such that the following holds, for all $\mathfrak{e} \in\left(0, \mathfrak{e}_{0}\right]$ :

(1) $E$ is twice continuously differentiable and strictly convex on $\mathcal{B}_{\mathfrak{p}}$ and it attains its unique global minimum at zero, $E(\mathbf{0})=\inf \left\{E(\boldsymbol{p}): \boldsymbol{p} \in \mathbb{R}^{3}\right\}$.

(2) $E(\mathbf{0})$ is a twofold degenerate eigenvalue of $\mathcal{H}(\mathbf{0})$. The expectation value of the photon number operator in any corresponding eigenstate is finite.

(3) If $\boldsymbol{p} \in \mathcal{B}_{\mathfrak{p}} \backslash\{\mathbf{0}\}$, then $E(\boldsymbol{p})$ is not an eigenvalue of $\mathcal{H}(\boldsymbol{p})$.

Proof. The assertions are contained in Theorems 4.6 and 5.3, Corollary 6.4, and Theorem 6.5; see also (2.22). 
While, for non-vanishing $\boldsymbol{p} \in \mathcal{B}_{\mathfrak{p}}, E(\boldsymbol{p})$ is not an eigenvalue of $\mathcal{H}(\boldsymbol{p})$, it is an eigenvalue of a certain renormalized Hamiltonian denoted by $\widetilde{\mathcal{H}}_{\infty}(\boldsymbol{p})$. Let us briefly explain the construction of the latter operator and of associated representations (all objects will be re-introduced in Sect. 2 in a systematic fashion):

We introduce a sequence of regularized vector potentials cut-off in the infra-red at $\rho_{j}:=\kappa / 2^{j}$,

$$
\boldsymbol{A}_{j}:=\sum_{\lambda \in \mathbb{Z}_{2}} \int_{\rho_{j} \leqslant|\boldsymbol{k}|<\kappa} \frac{\boldsymbol{\varepsilon}(\boldsymbol{k}, \lambda)}{(2|\boldsymbol{k}|)^{1 / 2}}\left(a^{\dagger}(\boldsymbol{k}, \lambda)+a(\boldsymbol{k}, \lambda)\right) \frac{\mathrm{d}^{3} \boldsymbol{k}}{(2 \pi)^{3 / 2}},
$$

where $j \in \mathbb{N}_{0}$. We further define regularized fiber Hamiltonians by

$$
\mathcal{H}_{j}^{\infty}(\boldsymbol{p}):=\sqrt{\left(\boldsymbol{\sigma} \cdot\left(\boldsymbol{p}-\mathbf{p}_{\mathrm{f}}+\mathfrak{e} \boldsymbol{A}_{j}\right)\right)^{2}+\mathbb{1}}+H_{\mathrm{f}} .
$$

(The purpose of the superscript $\infty$ is to keep the notation consistent with the one introduced in Sect. 2). Combining Lemma 3.3, Theorem 3.5, and (2.22) below we shall see that, for all $\boldsymbol{p} \in \mathcal{B}_{\mathfrak{p}}$, the Hamiltonian $\mathcal{H}_{j}^{\infty}(\boldsymbol{p})$ has an exactly twofold degenerate ground state eigenvalue. We denote the corresponding ground state eigenprojection by $\Pi_{j}^{\infty}(\boldsymbol{p})$. While $\mathcal{H}_{j}^{\infty}(\boldsymbol{p})$ converges to $\mathcal{H}(\boldsymbol{p})$ in the norm resolvent sense, the projections $\Pi_{j}^{\infty}(\boldsymbol{p})$ cannot have a nontrivial norm-limit by Theorem 1.1(3). To analyze this situation in more detail we proceed as follows:

Let $\mathscr{F}_{j}$ denote the bosonic Fock space over $L^{2}\left[\left\{|\boldsymbol{k}| \geqslant \rho_{j}\right\} \times \mathbb{Z}_{2}\right]$ and let $\mathscr{F}_{j}^{\infty}$ be the one over $L^{2}\left[\left\{|\boldsymbol{k}|<\rho_{j}\right\} \times \mathbb{Z}_{2}\right]$. In view of the natural embeddings $\mathscr{F}_{j} \subset \mathscr{F}_{j+1} \subset \mathscr{F}$ and the isomorphisms $\mathscr{F}_{j} \otimes \mathscr{F}_{j}^{\infty}=\mathscr{F}$ it makes sense to define the $C^{*}$-algebra, $\mathfrak{A}$, of local observables by

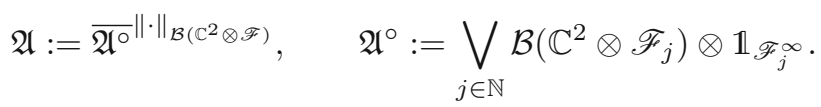

$(\mathcal{B}(\mathfrak{h})$ denotes the set of bounded operators on a Hilbert space $\mathfrak{h}$.) In physical terms the observables in $\mathfrak{A}^{\circ}$ are unable to detect photon momenta below a certain positive threshold value. To come back to our analysis of ground state projections we define a sequence of states, $\omega_{j, \boldsymbol{p}}$, on $\mathfrak{A}$ by

$$
\omega_{j, \boldsymbol{p}}(X):=\frac{1}{2} \operatorname{Tr}\left\{\Pi_{j}^{\infty}(\boldsymbol{p}) X\right\}, \quad X \in \mathfrak{A} .
$$

It will turn out that, unlike the projections $\Pi_{j}^{\infty}(\boldsymbol{p})$ themselves, the states $\omega_{j, \boldsymbol{p}}$ do have a limit, say $\omega_{\boldsymbol{p}}$, and the convergence takes place not only in the weak-* sense, but even in the norm on the dual space of $\mathfrak{A}$. These remarks apply to all $\boldsymbol{p} \in \mathcal{B}_{\mathfrak{p}}$.

In a particular coherent $I R$ representation of $\mathfrak{A}$ the limit $\omega_{\boldsymbol{p}}$ turns out to be normal. In fact, we shall find unitary Bogoliubov transformations, $W_{j}(\boldsymbol{p}) \in$ $\mathcal{B}\left(\mathbb{C}^{2} \otimes \mathscr{F}_{j}\right) \otimes \mathbb{1}_{\mathscr{F}_{j}^{\infty}}$, called dressing transformations in this context, having the following properties: They are consistent in the sense that

$$
W_{j}(\boldsymbol{p}) X W_{j}(\boldsymbol{p})^{*}=W_{m}(\boldsymbol{p}) X W_{m}(\boldsymbol{p})^{*}, \quad X \in \mathcal{B}\left(\mathbb{C}^{2} \otimes \mathscr{F}_{m}\right) \otimes \mathbb{1}_{\mathscr{F}_{m}^{\infty}}, m \leqslant j .
$$


As a consequence the relations

$$
\pi_{\boldsymbol{p}}(X):=W_{j}(\boldsymbol{p}) X W_{j}(\boldsymbol{p})^{*}, \quad X \in \mathcal{B}\left(\mathbb{C}^{2} \otimes \mathscr{F}_{m}\right) \otimes \mathbb{1}_{\mathscr{F}}^{\infty}, m \leqslant j,
$$

and a continuous extension define a $*$-representation $\pi_{p}: \mathfrak{A} \rightarrow \mathcal{B}\left(\mathbb{C}^{2} \otimes \mathscr{F}\right)$. Let

$$
\widetilde{\mathcal{H}}_{j}^{\infty}(\boldsymbol{p}):=W_{j}(\boldsymbol{p}) \mathcal{H}_{j}^{\infty}(\boldsymbol{p}) W_{j}(\boldsymbol{p})^{*} .
$$

Then our sequence of dressing transformations further satisfies

$$
\widetilde{\mathcal{H}}_{\infty}(\boldsymbol{p}):=\lim _{j \rightarrow \infty} \widetilde{\mathcal{H}}_{j}^{\infty}(\boldsymbol{p}) \text { exists in the norm resolvent sense. }
$$

In particular, $E(\boldsymbol{p})=\inf \operatorname{Spec}\left(\widetilde{\mathcal{H}}_{\infty}(\boldsymbol{p})\right)$. Finally, we can prove the following main theorem which partly summarizes some of the above remarks. In its statement we abbreviate

$$
\widetilde{\Pi}_{j}^{\infty}(\boldsymbol{p}):=W_{j}(\boldsymbol{p}) \Pi_{j}^{\infty}(\boldsymbol{p}) W_{j}(\boldsymbol{p})^{*}, \quad \widetilde{\Pi}_{\infty}(\boldsymbol{p}):=\mathbb{1}_{\{E(\boldsymbol{p})\}}\left(\widetilde{\mathcal{H}}_{\infty}(\boldsymbol{p})\right) .
$$

Theorem 1.2. For all $\kappa, \mathfrak{p}>0$, we find $\mathfrak{c}, \mathfrak{e}_{0}>0$ such that the following holds, for all $\boldsymbol{p} \in \mathcal{B}_{\mathfrak{p}}$ and $\mathfrak{e} \in\left(0, \mathfrak{e}_{0}\right]$ :

There exists a sequence of Bogoliubov transformations, $W_{j}(\boldsymbol{p})$, on $\mathbb{C}^{2} \otimes \mathscr{F}$ satisfying $W_{j}(\mathbf{0})=\mathbb{1},(1.7)$, and (1.8) such that

(1) $E(\boldsymbol{p})$ is an exactly twofold degenerate eigenvalue of $\widetilde{\mathcal{H}}_{\infty}(\boldsymbol{p})$, and

$$
\left\|\widetilde{\Pi}_{j}^{\infty}(\boldsymbol{p})-\widetilde{\Pi}_{\infty}(\boldsymbol{p})\right\| \leqslant \mathfrak{c} \mathfrak{e}(1+\mathfrak{c} \mathfrak{e})^{j} \rho_{j} \stackrel{j \rightarrow \infty}{\longrightarrow} 0 .
$$

(2) There is a state $\omega_{\boldsymbol{p}} \in \mathfrak{A}^{*}$ such that

$$
\omega_{j, \boldsymbol{p}} \rightarrow \omega_{\boldsymbol{p}} \quad \text { in } \mathfrak{A}^{*} \quad \text { and } \quad \omega_{\boldsymbol{p}}(X)=\frac{1}{2} \operatorname{Tr}\left\{\widetilde{\Pi}_{\infty}(\boldsymbol{p}) \pi_{\boldsymbol{p}}(X)\right\}, \quad X \in \mathfrak{A} .
$$

(3) If $\boldsymbol{q} \in \mathcal{B}_{\mathfrak{p}} \backslash\{\boldsymbol{p}\}$, then the representations $\pi_{\boldsymbol{p}}$ and $\pi_{\boldsymbol{q}}$ of $\mathfrak{A}$ are disjoint and, in particular, $\omega_{\boldsymbol{p}}$ is not a $\pi_{\boldsymbol{q}}$-normal state on $\mathfrak{A}$.

Proof. (1) follows from Corollary 4.5, Theorem 5.3, (2.22), and (2.53). (2) follows from Part (1) and the constructions explained above. (3) follows from Corollary 7.1 .

Explicit formulas for the dressing transformations $W_{j}(\boldsymbol{p})$ can be found in Sects. 2.4 and 2.5 .

\subsection{Organization of the Paper}

The remainder of this paper is organized as follows:

- In Sect. 2 the most important definitions are collected and their relevance is indicated. Sect. 2 is divided into six subsections:

2.1. The concept of second quantization in Fock space is recalled. The scales underlying the IAPT are introduced.

2.2. Technically convenient "doubled" Hilbert spaces and fiber Dirac operators are introduced.

2.3. IR regularized Hamiltonians $H_{m}^{j}(\boldsymbol{p})$ (acting in the doubled Hilbert spaces) and related objects are defined.

2.4. Objects necessary to prove regularity of the mass shell, in particular, "partially" dressing transformed operators $\check{H}_{j}(\boldsymbol{p})$ are introduced. 
2.5. "Fully" dressing transformed Hamiltonians $\widetilde{H}_{m}^{j}(\boldsymbol{p})$ are introduced.

2.6. Coherent IR representations $\pi_{p}$ are defined.

2.7. A general class of Hamiltonians $K_{m}^{j}$ is introduced which contains $H_{m}^{j}(\boldsymbol{p}), \check{H}_{j}(\boldsymbol{p})$, and $\widetilde{H}_{m}^{j}(\boldsymbol{p})$ as special cases.

- Section 3, devoted to preliminary results on the mass shell, is divided into three subsections:

3.1. The ground state energy and the spectral gap of Hamiltonians restricted to the initial scale are discussed.

3.2. Some a priori results on the mass shell are discussed (without regress to the IAPT).

3.3. Spectral gaps are bounded from below. This subsection is one of the key ingredients of the IAPT.

- Section 4 deals with the $C^{2}$-regularity and strict convexity of the mass shell. It has two subsections:

4.1. In two preparatory lemmas we state estimates crucially used in the proof of the regularity. Both are proved in Appendix C.

4.2. The $C^{2}$-regularity of the mass shell is proved.

- In Sect. 5 the degeneracy of ground state eigenvalues is investigated.

- The absence of ground state eigenvectors in the Fock representation for $\boldsymbol{p} \neq \mathbf{0}$ is proved in Sect. 6. The proof uses a formula for $a(k)$ applied to a ground state eigenvector of $H_{m}^{j}(\boldsymbol{p})$ derived in Appendix D.

- In Sect. 7 the disjointness of the representations $\pi_{p}$ is proved.

The main text is followed by four appendices:

A. Self-adjointness of fiber Dirac operators and Hamiltonians is discussed. Basic relative bounds involving various Hamiltonians are provided.

B. Analyticity of $\boldsymbol{p} \mapsto H_{m}^{j}(\boldsymbol{p})$ is discussed. Hellmann-Feynman formulas and bounds on the derivatives of $H_{m}^{j}(\boldsymbol{p})$ are derived.

C. The proofs of two technical lemmas needed in Sect. 4 are given.

D. A formula for $a(k)$ applied to a ground state of $H_{m}^{j}(\boldsymbol{p})$ is derived.

\section{Definitions, Notation, and Outlines}

\subsection{Scales, Fock Spaces, and Second Quantization}

The IAPT comprises a recursive application of perturbation theory to successively increase the range of photon momenta taken into account in the interaction part of the Hamiltonian, starting from the non-interacting situation. The iteration steps are labeled by $j \in \mathbb{N}_{0}=\{0,1,2, \ldots\}$ and associated with infra-red cut-offs $\rho_{j}$, defined by

$$
\rho_{j}:=\kappa(1 / 2)^{j}, \quad j \in \mathbb{N}_{0}, \quad \rho_{\infty}:=0, \quad \rho_{-\infty}:=\infty .
$$

Here $\kappa>0$ is the ultra-violet cutoff parameter. Hence, the set of photon momenta is split into a sequence of annuli,

$$
\mathbb{R}^{3} \times \mathbb{Z}_{2}=\mathcal{A}_{0} \cup \bigcup_{j \in \mathbb{N}_{0}} \mathcal{A}_{j}^{j+1} \cup\{0\},
$$




$$
\begin{aligned}
\mathcal{A}_{m}^{j} & :=\left\{(\boldsymbol{k}, \lambda) \in \mathbb{R}^{3} \times \mathbb{Z}_{2}: \rho_{j} \leqslant|\boldsymbol{k}|<\rho_{m}\right\}, \\
\mathcal{A}_{j} & :=\left\{(\boldsymbol{k}, \lambda) \in \mathbb{R}^{3} \times \mathbb{Z}_{2}: \rho_{j} \leqslant|\boldsymbol{k}|\right\} .
\end{aligned}
$$

Notice that $\mathcal{A}_{j}=\mathcal{A}_{-\infty}^{j}$ and $\mathbb{R}^{3} \times \mathbb{Z}_{2}=\mathcal{A}_{\infty}=\mathcal{A}_{-\infty}^{\infty}$. The arguments of photon wave functions are denoted by $k=(\boldsymbol{k}, \lambda)$ where $\boldsymbol{k}$ is representing the momentum and $\lambda$ the polarization of a single photon. We will frequently use the following convention for the scalar product of $f, g \in L^{2}\left[\mathcal{A}_{m}^{j}\right]$ :

$$
\langle f \mid g\rangle:=\int_{\mathcal{A}_{m}^{j}} \overline{f(k)} g(k) \mathrm{d} k:=\sum_{\lambda \in \mathbb{Z}_{2}} \int_{\rho_{j} \leqslant|\boldsymbol{k}|<\rho_{m}} \overline{f(\boldsymbol{k}, \lambda)} g(\boldsymbol{k}, \lambda) \mathrm{d}^{3} \boldsymbol{k} .
$$

The bosonic Fock space over the one photon Hilbert space $L^{2}\left[\mathcal{A}_{m}^{j}\right]$ is defined as

$$
\mathscr{F}_{m}^{j}:=\mathbb{C} \oplus \bigoplus_{n=1}^{\infty} L_{\mathrm{s}}^{2}\left[\left(\mathcal{A}_{m}^{j}\right)^{n}\right], \quad m<j .
$$

Here, $L_{\mathrm{s}}^{2}\left[\left(\mathcal{A}_{m}^{j}\right)^{n}\right]$ denotes the totally symmetric, square integrable wave functions on $\left(\mathcal{A}_{m}^{j}\right)^{n}$ with respect to $d^{n} k$. The elements of $\mathscr{F}_{m}^{j}$ are sequences $\psi=\left\{\psi^{(n)}\right\}_{n=0}^{\infty}$ with $\psi^{(n)} \in L_{\mathrm{s}}^{2}\left[\left(\mathcal{A}_{m}^{j}\right)^{n}\right], n \in \mathbb{N}$, and $\psi^{(0)} \in \mathbb{C}$, satisfying

$$
\|\psi\|^{2}:=\left|\psi^{(0)}\right|^{2}+\sum_{n=1}^{\infty}\left\|\psi^{(n)}\right\|^{2}<\infty
$$

The vector $\Omega_{m}^{j}:=(1,0,0, \ldots) \in \mathscr{F}_{m}^{j}$ is called the vacuum (vector) in $\mathscr{F}_{m}^{j}$. The Fock space over $L^{2}\left[\mathcal{A}_{j}\right]$ is denoted by $\mathscr{F}_{j}:=\mathscr{F}_{-\infty}^{j}$, its vacuum is $\Omega_{j}$. Moreover, $\mathscr{F}:=\mathscr{F}_{-\infty}^{\infty}$ and $\Omega$ denote the Fock space over $L^{2}\left[\mathbb{R}^{3} \times \mathbb{Z}_{2}\right]$ and the corresponding vacuum, respectively.

Pointwise creation and annihilation operators are usually interpreted as operator-valued distributions, but we refrain from using this formalism and use $a(k) \psi$ with $\psi \in \mathscr{F}_{m}^{j}$ to denote a $k$-dependent sequence of wave functions defined, for almost every $k \in \mathcal{A}_{m}^{j}$, by $a(k) \psi=\left\{(a(k) \psi)^{(n)}\right\}_{n \in \mathbb{N}_{0}}$ with

$$
(a(k) \psi)^{(n)}\left(k_{1}, \ldots, k_{n}\right)=(n+1)^{1 / 2} \psi^{(n+1)}\left(k, k_{1}, \ldots, k_{n}\right),
$$

and $a(k) \Omega_{m}^{j}=0$. The (smeared) annihilation operator of a photon state $f \in$ $L^{2}\left[\mathcal{A}_{m}^{j}\right]$ is defined as

$$
a(f) \psi:=\int_{\mathcal{A}_{m}^{j}} \overline{f(k)} a(k) \psi \mathrm{d} k,
$$

on its maximal domain. It is densely defined and closed and the (smeared) creation operator is its adjoint, i.e. $a^{\dagger}(f):=a(f)^{*}$. Straightforward computations yield the canonical commutation relations ( $C C R$ ),

$$
\left[a^{\sharp}(f), a^{\sharp}(g)\right]=0, \quad\left[a(f), a^{\dagger}(g)\right]=\langle f \mid g\rangle \mathbb{1}, \quad f, g \in L^{2}\left[\mathcal{A}_{m}^{j}\right],
$$

on a suitable domain, where $a^{\sharp}$ is $a^{\dagger}$ or $a$. Recall that all these remarks cover the cases $\mathcal{A}_{j}=\mathcal{A}_{-\infty}^{j}$ and $\mathbb{R}^{3} \times \mathbb{Z}_{2}=\mathcal{A}_{-\infty}^{\infty}$. 
Another application of (sharp) annihilation operators is the representation of the quadratic forms of second quantized multiplication operators, $\mathrm{d} \Gamma(\varpi)$, given by measurable functions $\varpi: \mathcal{A}_{m}^{j} \rightarrow \mathbb{R}$,

$$
\langle\psi \mid \mathrm{d} \Gamma(\varpi) \psi\rangle=\int \varpi(k)\langle a(k) \psi \mid a(k) \psi\rangle \mathrm{d} k,
$$

for $\psi$ such that the integral exists. More precisely, the second quantization of a multiplication operator $\varpi$ is the infinite direct sum

$$
\mathrm{d} \Gamma(\varpi):=\bigoplus_{n=0}^{\infty} \mathrm{d} \Gamma^{(n)}(\varpi),
$$

where $\mathrm{d} \Gamma^{(0)}(\varpi):=0$, and $\mathrm{d} \Gamma^{(n)}(\varpi)$ is the maximal operator in $L_{\mathrm{s}}^{2}\left[\left(\mathcal{A}_{m}^{j}\right)^{n}\right]$ of multiplication with

$$
\left(k_{1}, \ldots, k_{n}\right) \mapsto \varpi\left(k_{1}\right)+\cdots+\varpi\left(k_{n}\right) .
$$

Writing $\omega(k):=|\boldsymbol{k}|$ and $\boldsymbol{m}(k)=\boldsymbol{k}$ we define

$$
\begin{aligned}
H_{\mathrm{f}}^{(m, j)}:=\mathrm{d} \Gamma\left(\omega\left\lceil_{\mathcal{A}_{m}^{j}}\right),\right. & H_{\mathrm{f}}^{(j)}:=\mathrm{d} \Gamma\left(\omega \uparrow_{\mathcal{A}_{j}}\right), \\
\mathbf{p}_{\mathrm{f}}^{(m, j)}:=\mathrm{d} \Gamma\left(\boldsymbol{m}\left\lceil_{\mathcal{A}_{m}^{j}}\right),\right. & \mathbf{p}_{\mathrm{f}}^{(j)}:=\mathrm{d} \Gamma\left(\boldsymbol{m}\left\lceil_{\mathcal{A}_{j}}\right),\right. \\
N_{\mathrm{f}}^{(m, j)}:=\mathrm{d} \Gamma\left(1\left\lceil_{\mathcal{A}_{m}^{j}}\right),\right. & N_{\mathrm{f}}^{(j)}:=\mathrm{d} \Gamma\left(1\left\lceil_{\mathcal{A}_{j}}\right) .\right.
\end{aligned}
$$

The operators in the first line are called field energy operators, the triplet in the second line denotes field momentum operators, and the operators appearing in third line are number operators. If the underlying Hilbert space is $\mathscr{F}$, then they are denoted by $H_{\mathrm{f}}, \mathbf{p}_{\mathrm{f}}$, and $N_{\mathrm{f}}$, respectively.

Moreover, the following relations hold:

$$
\begin{aligned}
\left\|a^{\dagger}(f) \psi\right\|^{2} & =\|a(f) \psi\|^{2}+\|f\|^{2}\|\psi\|^{2}, \\
\|a(f) \psi\| & \left.\leqslant\left\|\varpi^{-1 / 2} f\right\| \| \mathrm{d} \Gamma(\varpi\rceil_{\mathcal{A}_{m}^{j}}\right)^{1 / 2} \psi \| .
\end{aligned}
$$

The identity in the first line follows from the CCR, for $f \in L^{2}\left[\mathcal{A}_{m}^{j}\right]$ and $\psi \in$ $\operatorname{dom}\left(\left[N_{\mathrm{f}}^{(m, j)}\right]^{1 / 2}\right)$, which is a core for $a^{\dagger}(f)$ and $a(f)$. The bound in the second line is valid, for $\psi \in \operatorname{dom}\left(d \Gamma\left(\varpi \Gamma_{\mathcal{A}_{m}^{j}}\right)^{1 / 2}\right)$ and $f \in L^{2}\left[\mathcal{A}_{m}^{j}\right]$ with $\left\|\varpi^{-1 / 2} f\right\|<\infty$ and $\varpi>0$ a.e.

For $h \in L^{2}\left[\mathcal{A}_{m}^{j}\right]$, we define field operators, on $\operatorname{dom}\left(\left[N^{(m, j)}\right]^{1 / 2}\right)$ to start with, by

$$
\varphi(h):=2^{-1 / 2}\left(a^{\dagger}(h)+a(h)\right), \quad \varpi(h):=2^{-1 / 2}\left(i a^{\dagger}(h)-i a(h)\right) .
$$

The subspace of $\mathscr{F}_{m}^{j}$ consisting of finite sequences $\left(\psi^{(0)}, \ldots, \psi^{(n)}, 0,0, \ldots\right)$ is a core of analytic vectors for $\varphi(h)$ and $\varpi(h)$, which, therefore, have unique selfadjoint extensions, henceforth again denoted by the same symbols. We use the notation $\varphi(\boldsymbol{f}), a^{\dagger}(\boldsymbol{f})$, and $a(\boldsymbol{f})$, for $\boldsymbol{f}=\left(f_{1}, f_{2}, f_{3}\right) \in L^{2}\left[\mathcal{A}_{m}^{j}, \mathbb{C}^{3}\right]$, to denote triplets of operators,

$$
\varphi(\boldsymbol{f}):=\left(\varphi\left(f_{1}\right), \varphi\left(f_{2}\right), \varphi\left(f_{3}\right)\right), \quad a^{\sharp}(\boldsymbol{f}):=\left(a^{\sharp}\left(f_{1}\right), a^{\sharp}\left(f_{2}\right), a^{\sharp}\left(f_{3}\right)\right) .
$$

Finally, we sketch a construction of the frequently used isomorphism

$$
\mathscr{F}_{m}^{j}=\mathscr{F}_{m}^{r} \otimes \mathscr{F}_{r}^{j}, \quad m<r<j .
$$


For a given orthonormal basis $(\mathrm{ONB})\left\{f_{\nu}\right\}_{\nu \in \mathbb{N}}$ of $L^{2}\left[\mathcal{A}_{m}^{j}\right]$, the set

$$
\left\{\Omega_{m}^{j},\left(a^{\dagger}\left(f_{\nu_{1}}\right)\right)^{n_{1}} \cdots\left(a^{\dagger}\left(f_{\nu_{\alpha}}\right)\right)^{n_{\alpha}} \Omega_{m}^{j}: \alpha \in \mathbb{N}, n_{s}, \nu_{s} \in \mathbb{N}, s=1, \ldots, \alpha\right\}
$$

defines a complete orthogonal system of vectors in $\mathscr{F}_{m}^{j}$. Now, let $\left\{g_{\nu}\right\}_{\nu \in \mathbb{N}}$ be an ONB of $L^{2}\left[\mathcal{A}_{m}^{r}\right]$ and let $\left\{h_{\mu}\right\}_{\mu \in \mathbb{N}}$ be an ONB of $L^{2}\left[\mathcal{A}_{r}^{j}\right]$. Clearly, $\left\{g_{\nu}, h_{\mu}\right\}_{\nu, \mu \in \mathbb{N}}$ defines an ONB of $L^{2}\left[\mathcal{A}_{m}^{j}\right]$. Thus, there is a unique isometric isomorphism between $\mathscr{F}_{m}^{j}$ and $\mathscr{F}_{m}^{r} \otimes \mathscr{F}_{r}^{j}$ satisfying

$$
\begin{aligned}
& \left(a^{\dagger}\left(g_{\nu_{1}}\right)\right)^{n_{1}} \cdots\left(a^{\dagger}\left(g_{\nu_{\alpha}}\right)\right)^{n_{\alpha}}\left(a^{\dagger}\left(h_{\mu_{1}}\right)\right)^{m_{1}} \cdots\left(a^{\dagger}\left(h_{\mu_{\beta}}\right)\right)^{m_{\beta}} \Omega_{m}^{j} \\
& \quad \mapsto\left(a^{\dagger}\left(g_{\nu_{1}}\right)\right)^{n_{1}} \cdots\left(a^{\dagger}\left(g_{\nu_{\alpha}}\right)\right)^{n_{\alpha}} \Omega_{m}^{r} \otimes\left(a^{\dagger}\left(h_{\mu_{1}}\right)\right)^{m_{1}} \cdots\left(a^{\dagger}\left(h_{\mu_{\beta}}\right)\right)^{m_{\beta}} \Omega_{r}^{j} .
\end{aligned}
$$

\subsection{Doubled Hilbert Spaces and Dirac Operators}

Recall that the Hilbert space the operators $\mathcal{H}(\boldsymbol{p})$ and $\mathcal{H}_{j}^{\infty}(\boldsymbol{p})$ with $\boldsymbol{p} \in \mathbb{R}^{3}$ are acting in is $\mathbb{C}^{2} \otimes \mathscr{F}$. For technical reasons it is, however, convenient to perform calculations in the doubled space $\mathbb{C}^{4} \otimes \mathscr{F}$ where we can represent square-roots as absolute values of Dirac operators. In this subsection we introduce the corresponding notation.

First, we introduce the Hilbert spaces

$$
\mathscr{H}_{j}:=\mathbb{C}^{4} \otimes \mathscr{F}_{j}, \quad j \in \mathbb{N}_{0} \cup\{\infty\}, \quad \mathscr{H}:=\mathscr{H}_{\infty} .
$$

Many calculations will be performed on the dense subspace $\mathscr{C}_{j}$ given by

$$
\begin{aligned}
\mathscr{C}_{j}:=\mathbb{C}^{4} \otimes\left\{\psi \in \mathscr{F}_{j}: \psi=\left(\psi^{(0)}, \ldots, \psi^{(n)}, 0,0, \ldots\right), \text { for some } n \in \mathbb{N},\right. \\
\left.\psi^{(i)} \text { has compact support for every } i \in \mathbb{N}\right\} .
\end{aligned}
$$

The $4 \times 4$-Dirac matrices,

$$
\alpha_{0}:=\left(\begin{array}{cc}
\mathbb{1} & 0 \\
0 & -\mathbb{1}
\end{array}\right), \quad \alpha_{j}:=\left(\begin{array}{cc}
0 & \sigma_{j} \\
\sigma_{j} & 0
\end{array}\right), \quad j=1,2,3,
$$

satisfy $\alpha_{i}=\alpha_{i}^{*}=\alpha_{i}^{-1}$ and the Clifford algebra relations

$$
\left\{\alpha_{i}, \alpha_{j}\right\}=2 \delta_{i j} \mathbb{1}, \quad i, j \in\{0,1,2,3\} .
$$

We recall that the $2 \times 2$-Pauli matrices appearing in (2.13) are given by

$$
\sigma_{1}=\left(\begin{array}{cc}
0 & 1 \\
1 & 0
\end{array}\right), \quad \sigma_{2}=\left(\begin{array}{cc}
0 & -i \\
i & 0
\end{array}\right), \quad \sigma_{3}=\left(\begin{array}{cc}
1 & 0 \\
0 & -1
\end{array}\right) .
$$

As a consequence of (2.14) and the $C^{*}$-equality we obtain

$$
\|\boldsymbol{\alpha} \cdot \boldsymbol{v}\|=|\boldsymbol{v}|, \quad \boldsymbol{v} \in \mathbb{R}^{3},
$$

where $\boldsymbol{\alpha} \cdot \boldsymbol{v}:=\alpha_{1} v_{1}+\alpha_{2} v_{2}+\alpha_{3} v_{3}$. With regard to (1.1) we define coupling functions

$$
\boldsymbol{G}_{m}(k):=\mathbb{1}_{\mathcal{A}_{m}}(k) \boldsymbol{G}(k), \quad \boldsymbol{G}(k):=(2 \pi)^{-3 / 2} \mathbb{1}_{|\boldsymbol{k}|<\kappa}|\boldsymbol{k}|^{-1 / 2} \boldsymbol{\varepsilon}(k),
$$

where $m \in \mathbb{N}_{0} \cup\{\infty\}$. Let $\boldsymbol{p} \in \mathbb{R}^{3}$ and let the fiber Dirac operator, $D_{m}^{j}(\boldsymbol{p})$, be defined as the closure of the symmetric operator in $\mathscr{H}_{j}$ given by

$$
D_{m}^{j}(\boldsymbol{p}) \psi:=\boldsymbol{\alpha} \cdot\left(\boldsymbol{p}-\mathbf{p}_{\mathrm{f}}^{(j)}+\mathfrak{e} \varphi\left(\boldsymbol{G}_{m}\right)\right) \psi+\alpha_{0} \psi, \quad \psi \in \operatorname{dom}\left(H_{\mathrm{f}}^{(j)}\right) .
$$


Let us remark at this point that, most of the time, we shall drop the arguments $(\boldsymbol{p})$ in the notation for all operators introduced here and below to avoid cluttered notation; i.e. we write $D_{m}^{j} \equiv D_{m}^{j}(\boldsymbol{p})$, etc. We keep the explicit reference to $\boldsymbol{p}$ in the notation only when it becomes important and also in most definitions to clarify the dependence on $\boldsymbol{p}$ of the new objects.

According to Lemma A.1 below $D_{m}^{j}$ is self-adjoint and $\mathscr{C}_{j}$ is a core for $D_{m}^{j}$. Moreover, we give an argument in Lemma A.1 which also shows that its square, $\left(D_{m}^{j}\right)^{2}$, is essentially self-adjoint on $\mathscr{C}_{j}$.

A direct computation shows, however, that

$$
\left(D_{m}^{j}\right)^{2}=\mathcal{T}_{m}^{j} \oplus \mathcal{T}_{m}^{j} \quad \text { on } \mathscr{C}_{j},
$$

where the direct sum refers to the splitting of the spinor components $\mathbb{C}^{4}=$ $\mathbb{C}^{2} \oplus \mathbb{C}^{2}$, and where

$$
\mathcal{T}_{m}^{j} \equiv \mathcal{T}_{m}^{j}(\boldsymbol{p}):=\left(\boldsymbol{\sigma} \cdot\left(\boldsymbol{p}-\mathbf{p}_{\mathrm{f}}^{(j)}+\mathfrak{e} \varphi\left(\boldsymbol{G}_{m}\right)\right)\right)^{2}+1 .
$$

In particular,

$$
\operatorname{Spec}\left(D_{m}^{j}\right) \subset(-\infty,-1] \cup[1, \infty) .
$$

We denote the resolvent at $i y \in i \mathbb{R}$ and the sign function of the fiber Dirac operator by

$$
\begin{aligned}
R_{m}^{j}(i y) & \equiv R_{m}^{j}(\boldsymbol{p}, i y):=\left(D_{m}^{j}(\boldsymbol{p})-i y\right)^{-1} \\
S_{m}^{j} & \equiv S_{m}^{j}(\boldsymbol{p}):=D_{m}^{j}(\boldsymbol{p})\left|D_{m}^{j}(\boldsymbol{p})\right|^{-1}
\end{aligned}
$$

Invoking the essential self-adjointness of $\left(D_{m}^{j}\right)^{2}$ it is easy to see that two possibilities to make sense out of the square root in (1.4) yield the same operator: we may take the square root of the closure of $\mathcal{T}_{m}^{j}$ defined by means of the spectral theorem, or, equivalently, we may define the square root in (1.4) to be equal to the upper left (or lower right) $2 \times 2$ block of the block-diagonal operator $\left|D_{m}^{j}\right|$.

An advantage of working with Dirac operators is the linear dependence of $D_{m}^{j}$ on the field operators. At the same time we have a convenient integral representation of their sign functions in terms of their resolvents; see (2.58) below.

For shortness we write $D_{j}, S_{j}, R_{j}$, for the operators $D_{j}^{j}, S_{j}^{j}, R_{j}^{j}$.

\subsection{The Hamiltonians $\mathcal{H}_{m}^{j}$ and $\boldsymbol{H}_{m}^{j}$, Ground State Energies, and Spectral Gaps}

We define fiber Hamiltonians on four spinors by

$$
H_{m}^{j}(\boldsymbol{p}):=\left|D_{m}^{j}(\boldsymbol{p})\right|+H_{\mathrm{f}}^{(j)},
$$

which are self-adjoint on the domain

$$
\operatorname{dom}\left(H_{m}^{j}(\boldsymbol{p})\right):=\operatorname{dom}\left(H_{\mathrm{f}}^{(j)}\right) \subset \mathscr{H}_{j}
$$

by Lemma A.3. These operators are block diagonal,

$$
H_{m}^{j}(\boldsymbol{p})=\mathcal{H}_{m}^{j}(\boldsymbol{p}) \oplus \mathcal{H}_{m}^{j}(\boldsymbol{p}), \quad \mathcal{H}_{m}^{j}(\boldsymbol{p}):=\overline{\mathcal{T}}_{m}^{j}(\boldsymbol{p})^{1 / 2}+H_{\mathrm{f}}^{(j)} .
$$


Hence, for $j=\infty$, they are twofold copies of the operators acting on twospinors that appeared in Sect. 1.2. Clearly, it suffices to prove the analogs of Theorems 1.1 and 1.2 for the Hamiltonians $H_{m}^{\infty}$ acting in $\mathbb{C}^{4} \otimes \mathscr{F}$ instead of proving them for the operators $\mathcal{H}_{m}^{\infty}$ in $\mathbb{C}^{2} \otimes \mathscr{F}$.

Define the ground state energy and the spectral gap of $H_{m}^{j}(\boldsymbol{p})$ by

$$
\begin{aligned}
E_{m}^{j}(\boldsymbol{p}) & :=\inf \operatorname{Spec}\left[H_{m}^{j}(\boldsymbol{p})\right], \\
\operatorname{gap}_{m}^{j}(\boldsymbol{p}) & :=\inf \left(\operatorname{Spec}\left[H_{m}^{j}(\boldsymbol{p})-E_{m}^{j}(\boldsymbol{p})\right] \backslash\{0\}\right) .
\end{aligned}
$$

The ground state projection of $H_{m}^{j}(\boldsymbol{p})$ is denoted by

$$
\Pi_{m}^{j}(\boldsymbol{p}):=\mathbb{1}_{\left\{E_{m}^{j}(\boldsymbol{p})\right\}}\left(H_{m}^{j}(\boldsymbol{p})\right) ;
$$

i.e. by the same symbol we have used for the ground state projections of operators acting on two-spinors. This should not cause any confusion in what follows. For $\operatorname{Re} z>0$, we further define

$$
\mathcal{R}_{m}^{j}(\boldsymbol{p}, z):=\left(H_{m}^{j}(\boldsymbol{p})-E_{m}^{j}(\boldsymbol{p})+z\right)^{-1},
$$

and, in case $E_{m}^{j}(\boldsymbol{p})$ is an isolated point of the spectrum, we define a projected resolvent,

$$
\left(\mathcal{R}_{m}^{j}\right)^{\perp}(\boldsymbol{p}):=\left(H_{m}^{j}(\boldsymbol{p})\left(\Pi_{m}^{j}\right)^{\perp}(\boldsymbol{p})-E_{m}^{j}(\boldsymbol{p})\right)^{-1}\left(\Pi_{m}^{j}\right)^{\perp}(\boldsymbol{p}),
$$

where $P^{\perp}:=\mathbb{1}-P$, for any orthogonal projection $P$.

As agreed on in the previous subsection we shall usually drop all arguments $(\boldsymbol{p})$ in the notation; for instance, we shall write $E_{m}^{j}, \operatorname{gap}_{m}^{j}, H_{m}^{j}, \mathcal{R}_{m}^{j}(z)$ instead of $E_{m}^{j}(\boldsymbol{p}), \operatorname{gap}_{m}^{j}(\boldsymbol{p}), H_{m}^{j}(\boldsymbol{p}), \mathcal{R}_{m}^{j}(\boldsymbol{p}, z)$.

Similarly, as in the previous subsection, we further use $H_{j}, \mathcal{H}_{j}, \mathcal{R}_{j}(z)$, $\left(\mathcal{R}_{j}\right)^{\perp}, \Pi_{j}, E_{j}, \operatorname{gap}_{j}$ as shorthands for $H_{j}^{j}, \mathcal{H}_{j}^{j}, \mathcal{R}_{j}^{j}(z),\left(\mathcal{R}_{j}^{j}\right)^{\perp}, \Pi_{j}^{j}, E_{j}^{j}$, gap ${ }_{j}^{j}$.

Without any subscript we denote quantities associated with $\mathcal{A}_{-\infty}^{\infty}=\mathbb{R}^{3} \times$ $\mathbb{Z}_{2}$, that is, $H:=H_{\infty}, \mathcal{H}:=\mathcal{H}_{\infty}, E:=E_{\infty}$, etc.

To motivate the above definitions we note the following:

Remarks on Sect. 3. In the iteration steps of the IAPT one infers spectral information on $H_{j}$ from the corresponding knowledge about operators with a larger infra-red cutoff, $H_{m}, m<j$. It is trivial to analyze the initial operator in this sequence, $H_{0}$, as it contains no field operators, $\boldsymbol{G}_{0}=\mathbf{0}$; it is merely a multiplication operator; see Lemma 3.1. In principle one would like to compare $H_{m}$ and $H_{j}$ with $m<j$ directly. However, these operators are defined on different Hilbert spaces so that effectively none of the usual spectral methods can be applied. This problem is solved by introducing $H_{m}^{j}$ which contains the same field operators as $H_{m}$ but acts in the same Hilbert space as $H_{j}$, namely $\mathscr{H}_{j}$. To explain this more precisely we recall that

$$
\begin{aligned}
\mathscr{H}_{j} & =\mathscr{H}_{m} \otimes \mathscr{F}_{m}^{j}=\bigoplus_{n=0}^{\infty} \mathscr{X}_{n}^{(m, j)}, \\
\mathscr{X}_{n}^{(m, j)} & :=\mathscr{H}_{m} \otimes L_{\mathrm{s}}^{2}\left[\left(\mathcal{A}_{m}^{j}\right)^{n}\right], \quad n \in \mathbb{N}, \quad \mathscr{X}_{0}^{(m, j)}:=\mathscr{H}_{m} .
\end{aligned}
$$


It is essential to note that $H_{m}^{j}$ leaves this decomposition invariant. Moreover, by a natural identification, see for instance [32], we have $\mathscr{X}_{n}^{(m, j)}=$ $L_{\mathrm{s}}^{2}\left[\left(\mathcal{A}_{m}^{j}\right)^{n} ; \mathscr{H}_{m}\right]$, and

$$
\left.H_{m}^{j}(\boldsymbol{p})\right|_{\mathscr{X}_{n}^{(m, j)}}=\int_{\left(\mathcal{A}_{m}^{j}\right)^{n}}^{\oplus}\left(H_{m}(\boldsymbol{p}-\underline{\boldsymbol{k}})+\left|\boldsymbol{k}_{1}\right|+\cdots+\left|\boldsymbol{k}_{n}\right|\right) \mathrm{d} k_{1} \ldots \mathrm{d} k_{n}
$$

with $\underline{\boldsymbol{k}}:=\boldsymbol{k}_{1}+\cdots+\boldsymbol{k}_{n}$. Thus, we can deduce the spectrum of $H_{m}^{j}(\boldsymbol{p})$ from that of $H_{m}(\boldsymbol{p}-\underline{\boldsymbol{k}})$; see Lemma 3.3. As a result of Sect. 3, obtained by combining Lemma 3.3 and Theorem 3.5, we shall see that the ground state energy is an isolated, fourfold degenerate eigenvalue of $H_{m}^{j}(\boldsymbol{p})$, for all $m \leqslant j<\infty, \boldsymbol{p} \in \mathcal{B}_{\mathfrak{p}}$, and small $\mathfrak{e}>0$, and that

$$
\operatorname{gap}_{j}(\boldsymbol{p}) \geqslant \mathfrak{c} \rho_{j} \quad \text { and } \quad E_{m}(\boldsymbol{p})=E_{m}^{j}(\boldsymbol{p}) .
$$

Here the constant $\mathfrak{c}$ can be chosen independently of $j$ and $\mathfrak{e} \in\left(0, \mathfrak{e}_{0}\right]$, for some $\mathfrak{e}_{0}>0$. One of the consequences of this result used later on is the following:

By means of the formula (2.58) (below) for the absolute value of Dirac operators it is straightforward to prove type A analyticity of the family $\left\{H_{m}^{j}(\boldsymbol{p})\right\}_{\boldsymbol{p} \in \mathbb{R}^{3}}$; see Lemma B.1. For small $\mathfrak{e}$, this entails analyticity of $E_{m}^{j}, m \leqslant$ $j<\infty$, on the set $\mathcal{B}_{\mathfrak{p}}$ where it is an isolated eigenvalue of constant multiplicity, and it allows to express its derivatives by means of Hellmann-Feynman type formulas; see Lemma B.1:

$$
\begin{aligned}
& \partial_{\boldsymbol{h}} E_{m}^{j}=\operatorname{Tr}\left\{\Pi_{m}^{j}\left(\partial_{\boldsymbol{h}} H_{m}^{j}\right) \Pi_{m}^{j}\right\} / 4, \\
& \partial_{\boldsymbol{h}}^{2} E_{m}^{j}=\operatorname{Tr}\left\{\Pi_{m}^{j}\left(\partial_{\boldsymbol{h}}^{2} H_{m}^{j}\right) \Pi_{m}^{j}\right\} / 4-\left\|\left(\left(\mathcal{R}_{m}^{j}\right)^{\perp}\right)^{1 / 2}\left(\partial_{\boldsymbol{h}} H_{m}^{j}\right) \Pi_{m}^{j}\right\|_{\mathrm{HS}}^{2} / 2 .
\end{aligned}
$$

Here, $\|\cdot\|_{\text {HS }}$ denotes the Hilbert-Schmidt norm and $\partial_{\boldsymbol{h}}$ the derivative in direction $\boldsymbol{h} \in \mathbb{R}^{3}$. We should mention here that parts of the proofs in Sect. 3 are formulated for more general operators.

\subsection{The Partially Dressing Transformed Hamiltonians $\check{\boldsymbol{H}}_{\boldsymbol{j}}$.}

Since $E_{j}$ turns out to be analytic on $\mathcal{B}_{\mathfrak{p}}$ it suffices to show that

$$
\sum_{j=0}^{\infty} \sup _{\boldsymbol{p} \in \mathcal{B}_{\mathfrak{p}}}\left|\partial_{\boldsymbol{h}}^{\nu} E_{j+1}(\boldsymbol{p})-\partial_{\boldsymbol{h}}^{\nu} E_{j}(\boldsymbol{p})\right|<\infty, \quad \nu=0,1,2, \boldsymbol{h} \in \mathbb{R}^{3},
$$

to prove $C^{2}$-regularity of $E \equiv E_{\infty}$, which is the pointwise limit of $E_{j}$ (in fact, $H_{j}^{\infty}$ converges to $H$ in the norm-resolvent sense; see Lemma A.3). If we attempted to prove (2.29) by means of the Hellmann-Feynman formulas in (2.28) for $\partial_{\boldsymbol{h}}^{\nu} E_{j+1}$ and $\partial_{\boldsymbol{h}}^{\nu} E_{j}^{j+1}=\partial_{\boldsymbol{h}}^{\nu} E_{j}$ applied to the operators $H_{j+1}$ and $H_{j}^{j+1}$ (which both act in $\mathscr{H}_{j+1}$ ) we would encounter the problem of estimating the difference between $\Pi_{j}^{j+1}$ and $\Pi_{j+1}$. But if we expect Theorem 1.1(3) to be true, then the norms $\left\|\Pi_{j}^{j+1}-\Pi_{j+1}\right\|$ cannot be summable at $\boldsymbol{p} \neq \mathbf{0}$, for otherwise one could infer the existence of ground states of $H(\boldsymbol{p})$. In fact, anticipating a result of Lemma 3.3(1), we have

$$
\Pi_{j}^{j+1}=\Pi_{j} \otimes P_{\Omega_{j}^{j+1}} \in \mathcal{B}\left(\mathscr{H}_{j+1}\right)=\mathcal{B}\left(\mathscr{H}_{j}\right) \otimes \mathcal{B}\left(\mathscr{F}_{j}^{j+1}\right),
$$


where $P_{\Omega_{j}^{j+1}}$ is the one-dimensional projection onto the space spanned by $\Omega_{j}^{j+1}$ in $\mathscr{F}_{j}^{j+1}$. In other words, there are no photons in the range of $\Pi_{j}^{j+1}$ with momentum in $\mathcal{A}_{j}^{j+1}$. A moving electron, however, emits and absorbs radiation and is thought of being surrounded by a cloud of soft photons. So, if the total system is moving with a non-vanishing momentum $\boldsymbol{p}$, then $\Pi_{j}^{j+1}(\boldsymbol{p})$ will not be a good approximation to its ground state projection. Roughly speaking the idea is then to remedy this situation by introducing a unitary (partial) dressing transformation, $U_{j}^{*}(\boldsymbol{p})$, that dresses $\Omega_{j}^{j+1}$ into a cloud of soft photons.

Hence, we compare $H_{j}^{j+1}$ not with $H_{j+1}$, but with

$$
\check{H}_{j+1}:=U_{j} H_{j+1} U_{j}^{*}
$$

instead. The definition of the $\boldsymbol{p}$-dependent unitary $U_{j}$ is given in formula $(2.32)$ below. Clearly,

$$
\begin{aligned}
\operatorname{Spec}\left[\check{H}_{j+1}\right] & =\operatorname{Spec}\left[H_{j+1}\right], \quad E_{j+1}=\inf \operatorname{Spec}\left[\check{H}_{j+1}\right], \\
\operatorname{gap}_{j+1} & =\inf \left(\operatorname{Spec}\left[\check{H}_{j+1}-E_{j+1}\right] \backslash\{0\}\right),
\end{aligned}
$$

and the ground state projection of $\check{H}_{j+1}$ satisfies

$$
\check{\Pi}_{j+1}:=\mathbb{1}_{\left\{E_{j+1}\right\}}\left(\check{H}_{j+1}\right)=U_{j} \Pi_{j+1} U_{j}^{*} .
$$

By unitary invariance of the trace and the Hilbert-Schmidt norm we obtain from (2.28)

$$
\begin{aligned}
\partial_{\boldsymbol{h}} E_{j+1}= & \operatorname{Tr}\left\{\check{\Pi}_{j+1} U_{j}\left(\partial_{\boldsymbol{h}} H_{j+1}\right) U_{j}^{*} \check{\Pi}_{j+1}\right\} / 4 \\
\partial_{\boldsymbol{h}}^{2} E_{j+1}= & \operatorname{Tr}\left\{\check{\Pi}_{j+1} U_{j}\left(\partial_{\boldsymbol{h}}^{2} H_{j+1}\right) U_{j}^{*} \check{\Pi}_{j+1}\right\} / 4 \\
& -\left\|\left(\left(\check{\mathcal{R}}_{j+1}\right)^{\perp}\right)^{1 / 2} U_{j}\left(\partial_{\boldsymbol{h}} H_{j+1}\right) U_{j}^{*} \check{\Pi}_{j+1}\right\|_{\mathrm{HS}}^{2} / 2
\end{aligned}
$$

where $\left(\check{\mathcal{R}}_{j+1}\right)^{\perp}=U_{j}\left(\mathcal{R}_{j+1}\right)^{\perp} U_{j}^{*}$; see also (2.41) below. For $U_{j}(\boldsymbol{p})$ one chooses a Bogoliubov transformation of the form

$$
\begin{aligned}
U_{j}(\boldsymbol{p}) & :=e^{-i \mathfrak{e}\left(f_{j}^{j+1}(\boldsymbol{p})\right)}, \\
f_{j}^{j+1}(\boldsymbol{p}) & :=\frac{\boldsymbol{G}_{j}^{j+1} \cdot \nabla E_{j}(\boldsymbol{p})}{\omega-\boldsymbol{k} \cdot \nabla E_{j}(\boldsymbol{p})}, \quad \boldsymbol{G}_{j}^{j+1}:=\mathbb{1}_{\mathcal{A}_{j}^{j+1}} \boldsymbol{G} .
\end{aligned}
$$

We define $f_{j}^{j+1}(\boldsymbol{p})$ only for $\boldsymbol{p} \in \mathcal{B}_{\mathfrak{p}}$ and sufficiently small $\mathfrak{e}$. In fact, by Lemma 3.2, Theorem 3.5, and Lemma B.1 below,

$$
\sup _{\mathfrak{e} \in\left(0, \mathfrak{e}_{0}\right]} \sup _{j \in \mathbb{N}} \sup _{\mathcal{B}_{\mathfrak{p}}}\left|\nabla E_{j}\right| \leqslant \mathfrak{q}<1
$$

where $\mathfrak{e}_{0}$ and $\mathfrak{q}$ depend only on $\kappa$ and $\mathfrak{p}$.

It is well known that, for every real-valued $h \in L^{2}\left[\mathcal{A}_{j}^{j+1}, \mathbb{R}\right]$,

$$
U_{j} a^{\sharp}(h) U_{j}^{*}=a^{\sharp}(h)-\left\langle h \mid f_{j}^{j+1}\right\rangle, \quad U_{j} \varphi(h) U_{j}^{*}=\varphi(h)-2\left\langle h \mid f_{j}^{j+1}\right\rangle,
$$

on the domain of $\left[H_{\mathrm{f}}^{(j, j+1)}\right]^{1 / 2}$ (which stays invariant under $U_{j}$ ). So, $U_{j}$ actually gives rise to a new representation of the CCR. The explicit choice of $f_{j}^{j+1}$ can be motivated by the bound (6.3) below which reflects the IR behavior of the ground state eigenvectors of $H_{j}$. 
The action of $U_{j}$ on various operators can be expressed by means of the quantities

$$
\begin{aligned}
\boldsymbol{F}_{j}^{j+1} & :=f_{j}^{j+1} \boldsymbol{k}+\boldsymbol{G}_{j}^{j+1}, \quad \check{\boldsymbol{c}}_{j}:=\left\langle f_{j}^{j+1} \mid \boldsymbol{F}_{j}^{j+1}+\boldsymbol{G}_{j}^{j+1}\right\rangle, \\
\check{b}_{j} & :=\left\langle f_{j}^{j+1} \mid \omega f_{j}^{j+1}\right\rangle,
\end{aligned}
$$

which all depend on $\boldsymbol{p}$ through $f_{j}^{j+1}$. For later reference we note the bounds

$$
\left|\check{b}_{j}\right|,\left|\check{\boldsymbol{c}}_{j}\right| \leqslant \mathfrak{c} \rho_{j}, \quad\left\|\boldsymbol{F}_{j}^{j+1}\right\| \leqslant \mathfrak{c} \rho_{j}, \quad\left\|\omega^{-1 / 2} \boldsymbol{F}_{j}^{j+1}\right\| \leqslant \mathfrak{c} \rho_{j}^{1 / 2} .
$$

On account of (2.33) the bounds (2.34) hold true uniformly, for $j \in \mathbb{N}_{0}, \boldsymbol{p} \in \mathcal{B}_{\mathfrak{p}}$, and $\mathfrak{e} \in\left(0, \mathfrak{e}_{0}\right]$, with $\mathfrak{c}, \mathfrak{e}_{0}>0$ depending only on $\kappa$ and $\mathfrak{p}$. The following identity will lead to some crucial cancellations:

$$
\boldsymbol{F}_{j}^{j+1} \cdot \nabla E_{j}=\frac{1}{(2 \pi)^{3 / 2}} \frac{\mathbb{1}_{\mathcal{A}_{j}^{j+1}}}{\omega^{1 / 2}} \frac{\boldsymbol{\varepsilon} \cdot \nabla E_{j}}{1-\grave{k} \cdot \nabla E_{j}}=\omega f_{j}^{j+1}, \quad \stackrel{\circ}{\boldsymbol{k}}:=|\boldsymbol{k}|^{-1} \boldsymbol{k} .
$$

Abbreviating

$$
\check{D}_{j+1}:=U_{j} D_{j+1} U_{j}^{*}, \quad \check{H}_{\mathrm{f}}^{(j+1)}:=U_{j} H_{\mathrm{f}}^{(j+1)} U_{j}^{*}, \quad j \in \mathbb{N}_{0},
$$

we deduce the following explicit formulas for these operators:

$$
\begin{aligned}
\check{H}_{\mathrm{f}}^{(j+1)} & =H_{\mathrm{f}}^{(j+1)}-\mathfrak{e} \varphi\left(\omega f_{j}^{j+1}\right)+\mathfrak{e}^{2} \check{b}_{j}, \\
\check{D}_{j+1} & =D_{j}^{j+1}+\boldsymbol{\alpha} \cdot\left\{\mathfrak{e} \varphi\left(\boldsymbol{F}_{j}^{j+1}\right)-\mathfrak{e}^{2} \check{\boldsymbol{c}}_{j}\right\},
\end{aligned}
$$

which hold true on the domain of $H_{\mathrm{f}}^{(j+1)}$. The resolvents of $\check{D}_{j}$ and

$$
\check{H}_{j}=\left|\check{D}_{j}\right|+\check{H}_{\mathrm{f}}^{(j)}
$$

are tagged with a check on top, i.e. we set

$$
\begin{aligned}
\check{R}_{j}(i y) \equiv \check{R}_{j}(\boldsymbol{p}, i y) & :=\left(\check{D}_{j}(\boldsymbol{p})-i y\right)^{-1}, & y \in \mathbb{R}, \\
\check{\mathcal{R}}_{j}(z) \equiv \check{\mathcal{R}}_{j}(\boldsymbol{p}, z) & :=\left(\check{H}_{j}(\boldsymbol{p})-E_{j}(\boldsymbol{p})+z\right)^{-1}, & \operatorname{Re} z>0, \\
\check{\mathcal{R}}_{j}^{\perp}(z) \equiv \check{\mathcal{R}}_{j}^{\perp}(\boldsymbol{p}, z) & :=\left(\check{H}_{j}(\boldsymbol{p})-E_{j}(\boldsymbol{p})+z\right)^{-1} \check{\Pi}_{j}^{\perp}(\boldsymbol{p}), & \operatorname{Re} z>-\operatorname{gap}_{j}(\boldsymbol{p}),
\end{aligned}
$$$$
\check{\mathcal{R}}_{j}^{\perp}:=\check{\mathcal{R}}_{j}^{\perp}(\boldsymbol{p}, 0)=\left(\check{H}_{j}(\boldsymbol{p})-E_{j}(\boldsymbol{p})\right)^{-1} \check{\Pi}_{j}^{\perp}(\boldsymbol{p}) .
$$

The latter resolvents will only be used when $E_{j}$ is an isolated point of the spectrum.

Outline of Sect. 4.2. To give the reader an idea on where and how $\check{H}_{j+1}$ crucially enters into the analysis we add a few remarks on Sect. 4.2: A simple but lengthy calculation (carried out in Lemma 4.1) using (2.35) yields

$$
\begin{aligned}
& \left(\check{H}_{j+1}-H_{j}^{j+1}+\mathfrak{e}^{2} \varkappa_{j}\right) \Pi_{j}^{j+1} \\
& \quad=\mathfrak{e}\left(\Pi_{j}^{\perp} \nabla H_{j} \Pi_{j}\right) \otimes\left\{a^{\dagger}\left(\boldsymbol{F}_{j}^{j+1}\right)-\mathfrak{e} \check{\boldsymbol{c}}_{j}\right\} P_{\Omega_{j}^{j+1}}+\mathcal{O}\left(\mathfrak{e} \rho_{j}^{2}\right),
\end{aligned}
$$

where $\varkappa_{j} \geqslant 0$. Since $E_{j}$ belongs to the resolvent set of $\check{H}_{j+1} \check{\Pi}_{j+1}^{\perp}$ we further have

$$
\check{\Pi}_{j+1}^{\perp} \Pi_{j}^{j+1}=\left(\check{H}_{j+1}-E_{j}+t\right)^{-1} \check{\Pi}_{j+1}^{\perp}\left(\check{H}_{j+1}-H_{j}^{j+1}+t\right) \Pi_{j}^{j+1},
$$


for every $t \geqslant 0$. Using a "resolvent comparison lemma" (Lemma 4.2) and the two identities above we deduce in Lemma 4.3 that

$$
\left\|\check{\Pi}_{j+1}-\Pi_{j}^{j+1}\right\|_{\mathrm{HS}} \leqslant 4\left\|\check{\Pi}_{j+1}^{\perp} \Pi_{j}^{j+1}\right\|_{\mathrm{HS}} \leqslant \mathfrak{c} \mathfrak{e} \rho_{j} L_{j}^{(1)}+\mathcal{O}\left(\mathfrak{e} \rho_{j}\right),
$$

where

$$
L_{j}^{(s)}:=\left\|\left(\mathcal{R}_{j}^{\perp}\right)^{s} \Pi_{j}^{\perp} \nabla H_{j} \Pi_{j}\right\|_{\mathrm{HS}}, \quad j \in \mathbb{N}_{0}, s \in\{1 / 2,1\} .
$$

Notice that it is essential not to estimate the operator norm of the resolvent in (2.42) trivially in the leading order contribution to (2.42). Rather it has to be related to $\mathcal{R}_{j}^{\perp}$ by means of Lemma 4.2 . The square of $L_{j}^{(1 / 2)}$ is up to a trivial factor the second part of the Hellmann-Feynman formula (2.28) for $\partial_{\boldsymbol{h}}^{2} E_{j}$ and its control is, therefore, necessary to derive (2.29). We shall prove the following relations:

$$
L_{j+1}^{(1)} \leqslant(1+\mathfrak{c} \mathfrak{e})^{j+1}-1, \quad\left|L_{j+1}^{(1 / 2)}-L_{j}^{(1 / 2)}\right| \leqslant \mathfrak{c} \mathfrak{e} \rho_{j}^{1 / 2}(1+\mathfrak{c} \mathfrak{e})^{j}
$$

see Lemma 4.4 and Corollary 4.5. Estimating the resolvents in $L_{j}^{(s)}$ trivially,

$$
L_{j}^{(1)} \leqslant \mathfrak{c} \rho_{j}^{-1}, \quad L_{j}^{(1 / 2)} \leqslant \mathfrak{c} \rho_{j}^{-1 / 2},
$$

would again by far not be sufficient. It is a guiding theme in the IAPT to avoid such trivial bounds by relating singularities of resolvents recursively to those corresponding to a preceding scale. (In the initial scale $L_{0}^{(s)}=0$.) The final result of Sect. 4.2 on the $C^{2}$-regularity of the mass shell (Theorem 4.6) is based on the estimates (2.43) and (2.45).

\subsection{The Fully Dressing Transformed Hamiltonians $\tilde{\boldsymbol{H}}_{m}^{j}$}

In order to establish Theorem 1.2 we define the following dressing transforms:

$$
W_{m}^{j}(\boldsymbol{p}):=\prod_{\ell=m}^{j-1} U_{\ell}(\boldsymbol{p}), \quad m<j<\infty, \quad W_{m}:=W_{0}^{m} .
$$

We shall often identify $W_{m}^{j} \equiv W_{m}^{j} \otimes \mathbb{1}_{\mathscr{F}_{j}^{\ell}}$, if $m<j<\ell$. Of course, we assume here that $U_{\ell}$ is well defined, i.e. we assume that we are in the situation described in the paragraph below (2.32). Furthermore, we abbreviate

$$
\begin{array}{rlrl}
f_{m}(\boldsymbol{p}) & :=\sum_{\ell=0}^{m-1} f_{\ell}^{\ell+1}(\boldsymbol{p}), & \boldsymbol{F}_{m}(\boldsymbol{p}):=\sum_{\ell=0}^{m-1} \boldsymbol{F}_{\ell}^{\ell+1}(\boldsymbol{p}), \\
\widetilde{\boldsymbol{c}}_{m}(\boldsymbol{p}):=\left\langle f_{m}(\boldsymbol{p}) \mid \boldsymbol{G}_{m}+\boldsymbol{F}_{m}(\boldsymbol{p})\right\rangle, & \widetilde{b}_{m}(\boldsymbol{p}):=\left\langle f_{m}(\boldsymbol{p}) \mid \omega f_{m}(\boldsymbol{p})\right\rangle,
\end{array}
$$

where $m \in \mathbb{N}_{0} \cup\{\infty\},|\boldsymbol{p}| \leqslant \mathfrak{p}$, and empty sums are zero. Then we define

$$
\widetilde{D}_{m}^{j}(\boldsymbol{p}):=\boldsymbol{\alpha} \cdot\left\{\boldsymbol{p}-\mathbf{p}_{\mathrm{f}}^{(j)}+\mathfrak{e} \varphi\left(\boldsymbol{G}_{m}+\boldsymbol{F}_{m}(\boldsymbol{p})\right)-\mathfrak{e}^{2} \widetilde{\boldsymbol{c}}_{m}(\boldsymbol{p})\right\}+\alpha_{0},
$$

a priori as an essentially self-adjoint operator on $\mathscr{C}_{j}$, and then by taking its closure; see Lemma A.3. Furthermore, we define, on the $\operatorname{domain} \operatorname{dom}\left(H_{\mathrm{f}}^{(j)}\right)$,

$$
\widetilde{H}_{m}^{j}(\boldsymbol{p}):=\left|\widetilde{D}_{m}^{j}(\boldsymbol{p})\right|+H_{\mathrm{f}}^{(j)}-\mathfrak{e} \varphi\left(\omega f_{m}(\boldsymbol{p})\right)+\mathfrak{e}^{2} \widetilde{b}_{m}(\boldsymbol{p}) .
$$


In (2.48) and (2.49) we may choose $j, m \in \mathbb{N}_{0} \cup\{\infty\}$ with $m \leqslant j$. Notice, however, that

$$
\widetilde{H}_{m}^{j}=W_{m} H_{m}^{j} W_{m}^{*}, \quad \text { if } m<\infty,
$$

while there is no analog of (2.50) with $m=\infty$. In fact, it does not make sense to define $W_{\infty}$ by $(2.47)$ because $\sum_{0}^{\infty} f_{j}^{j+1} \notin L^{2}\left[\mathbb{R}^{3} \times \mathbb{Z}_{2}\right]$. In view of the identity

$$
\inf \operatorname{Spec}\left[\widetilde{H}_{m}^{j}\right]=E_{m}^{j},
$$

which is obvious for $m<\infty$ and follows from Lemma A.3(iii) in the case $m=j=\infty$, we set

$$
\widetilde{\Pi}_{m}^{j}(\boldsymbol{p}):=\mathbb{1}_{\left\{E_{m}^{j}(\boldsymbol{p})\right\}}\left(\widetilde{H}_{m}^{j}(\boldsymbol{p})\right) .
$$

In view of $(2.50)$ we then have

$$
\widetilde{\Pi}_{m}^{j}=W_{m} \Pi_{m}^{j} W_{m}^{*}, \quad \text { if } m<\infty .
$$

By virtue of Lemma 3.3(1) and Theorem 3.5 below we shall see that

$$
\Pi_{j}^{\infty}=\Pi_{j} \otimes P_{\Omega_{j}^{\infty}}=\Pi_{j}^{j+1} \otimes P_{\Omega_{j+1}^{\infty}}, \quad j \in \mathbb{N}_{0} .
$$

Hence, the ground state projections defined so far are related as

$$
\begin{aligned}
\widetilde{\Pi}_{j+1}^{\infty}-\widetilde{\Pi}_{j}^{\infty} & =W_{j}\left(U_{j} \Pi_{j+1} U_{j}^{*}-\Pi_{j}^{j+1}\right) W_{j}^{*} \otimes P_{\Omega_{j+1}^{\infty}} \\
& =W_{j}\left(\check{\Pi}_{j+1}-\Pi_{j}^{j+1}\right) W_{j}^{*} \otimes P_{\Omega_{j+1}^{\infty}} .
\end{aligned}
$$

In particular, (2.43) combined with (2.45) implies the convergence of $\widetilde{\Pi}_{j}^{\infty}$ to some limit projection, provided that $\mathfrak{e}>0$ is sufficiently small. This limit projection is discussed in Sect. 5 .

\subsection{Coherent IR Representations}

We define an algebra analog to that in the introduction,

$$
\mathfrak{A}:=\overline{\mathfrak{A}}^{\|\cdot\|_{\mathfrak{B}(\mathscr{H})}}, \quad \mathfrak{A}^{\circ}:=\bigvee_{j \in \mathbb{N}} \mathfrak{B}\left(\mathscr{H}_{j}\right) \otimes \mathbb{1}_{\mathscr{F}_{j}^{\infty}} .
$$

(It differs from the one in (1.5) only in that we now consider Hilbert spaces of four-spinors instead of two-spinors. We shall use the same notation for algebras and representations in both cases. This should not lead to any confusion). In $(2.54)$ we have used the identifications $\mathscr{H}=\mathscr{H}_{j} \otimes \mathscr{F}_{j}^{\infty}$ and $\mathfrak{B}(\mathscr{H})=$ $\mathfrak{B}\left(\mathscr{H}_{j}\right) \otimes \mathfrak{B}\left(\mathscr{F}_{j}^{\infty}\right)$, which follow from (2.10). The coherent representations are the unital $*$-representations $\pi_{\boldsymbol{p}}: \mathfrak{A} \rightarrow \mathfrak{B}(\mathscr{H})$ determined, for each $\boldsymbol{p} \in \mathcal{B}_{\mathfrak{p}}$ and sufficiently small $\mathfrak{e}$, by

$$
\pi_{\boldsymbol{p}}\left(X \otimes \mathbb{1}_{\mathscr{F}_{j}^{\infty}}\right):=W_{j} X W_{j}^{*} \otimes \mathbb{1}_{\mathscr{F}_{j}^{\infty}}, \quad X \in \mathfrak{B}\left(\mathscr{H}_{j}\right), j \in \mathbb{N} .
$$

Note that we have the consistency relations (1.7) so that $\pi_{\boldsymbol{p}}$ is well defined and isometric on $\mathfrak{A}^{\circ}$. It, therefore, has a unique isometric extension to the whole $C^{*}$-algebra $\mathfrak{A}$ which is again denoted by $\pi_{\boldsymbol{p}}$. 
Since we are now dealing with four-spinors instead of two-spinors we define the states $\omega_{j, \boldsymbol{p}}$ by

$$
\omega_{j, \boldsymbol{p}}(X):=\frac{1}{4} \operatorname{Tr}\left\{\Pi_{j}^{\infty}(\boldsymbol{p}) X\right\}, \quad X \in \mathfrak{A} .
$$

Of course, we shall employ this definition only in a situation where we already know that $\Pi_{j}^{\infty}(\boldsymbol{p})$ has rank four. On account of the invariance of the trace with respect to unitary conjugations we obtain, for $m<j$ and $X \in \mathfrak{B}\left(\mathscr{H}_{m}\right)$,

$$
\omega_{j, \boldsymbol{p}}\left(X \otimes \mathbb{1}_{\mathscr{F} m}\right)=\frac{1}{4} \operatorname{Tr}\left\{\Pi_{j}^{\infty} X \otimes \mathbb{1}_{\mathscr{F}_{m}^{\infty}}\right\}=\frac{1}{4} \operatorname{Tr}\left\{\widetilde{\Pi}_{j}^{\infty} \pi_{\boldsymbol{p}}\left(X \otimes \mathbb{1}_{\mathscr{F}_{m}^{\infty}}\right)\right\} .
$$

In view of $(2.43),(2.45)$, and $(2.53)$ we thus have convergence of $\omega_{j, \boldsymbol{p}}$ to some state $\omega_{p}$ with respect to the norm on $\mathfrak{A}^{*}$.

The disjointness of representations $\pi_{\boldsymbol{p}}$ corresponding to distinct $\boldsymbol{p}$ is shown in Sect. 7.

\subsection{Generalized Operators}

We define generalized operators $K_{m}^{j}$ which cover the special cases

$$
\left\{H_{m}^{j}(\boldsymbol{p})\right\}_{m \leqslant j}, \quad\left\{\check{H}_{j}(\boldsymbol{p})\right\}_{j}, \quad \text { and } \quad\left\{\widetilde{H}_{m}^{j}(\boldsymbol{p})\right\}_{m \leqslant j} .
$$

In what follows we pick $\boldsymbol{g} \in L^{2}\left[\mathcal{A}_{0}^{\infty}, \mathbb{R}^{3}\right], h \in L^{2}\left[\mathcal{A}_{0}^{\infty}, \mathbb{R}\right]$, and $\boldsymbol{c}_{m} \in \mathbb{R}^{3}, b_{m} \in$ $\mathbb{R}, m \in \mathbb{N}$, and set $\boldsymbol{c}_{0}:=\mathbf{0}, b_{0}:=0$, and for $m<j$,

$$
\begin{aligned}
& \boldsymbol{c}_{m}^{j}:=\boldsymbol{c}_{j}-\boldsymbol{c}_{m}, \quad b_{m}^{j}:=b_{j}-b_{m}, \\
& \boldsymbol{g}_{m}^{j}:=\mathbb{1}_{\mathcal{A}_{m}^{j}} \boldsymbol{g}, \quad h_{m}^{j}:=\mathbb{1}_{\mathcal{A}_{m}^{j}} h, \quad \boldsymbol{g}_{m}:=\boldsymbol{g}_{0}^{m}, \quad h_{m}:=h_{0}^{m} .
\end{aligned}
$$

On the dense domain $\mathscr{C}_{j}$ [introduced in (2.12)] we define

$$
\begin{aligned}
& \mathfrak{D}_{m}^{j}(\boldsymbol{p}):=\boldsymbol{\alpha} \cdot\left(\boldsymbol{p}-\mathbf{p}_{\mathrm{f}}^{(j)}+\mathfrak{e} \varphi\left(\boldsymbol{g}_{m}\right)-\mathfrak{e}^{2} \boldsymbol{c}_{m}\right)+\alpha_{0}, \\
& K_{m}^{j}(\boldsymbol{p}):=\left|\mathfrak{D}_{m}^{j}(\boldsymbol{p})\right|+H_{\mathrm{f}}^{(j)}-\mathfrak{e} \varphi\left(h_{m}\right)+\mathfrak{e}^{2} b_{m} .
\end{aligned}
$$

Notice that $\mathfrak{D}_{m}^{j}(\boldsymbol{p})$ is essentially self-adjoint on $\mathscr{C}_{j}$ by Lemma A.1. Denoting its self-adjoint closure again by the same symbol we have $\left(\mathfrak{D}_{m}^{j}(\boldsymbol{p})\right)^{2} \geqslant 1$ and we define

$$
\mathfrak{R}_{m}^{j}(\boldsymbol{p}, i y):=\left(\mathfrak{D}_{m}^{j}(\boldsymbol{p})-i y\right)^{-1}, \quad y \in \mathbb{R} .
$$

As 0 belongs to the resolvent set of $\mathfrak{D}_{m}^{j}$ its sign function can be represented as a strongly convergent principal value (see, e.g., [21, Page 359]),

$$
\mathcal{S}_{m}^{j}(\boldsymbol{p}) \psi:=\mathfrak{D}_{m}^{j}(\boldsymbol{p})\left|\mathfrak{D}_{m}^{j}(\boldsymbol{p})\right|^{-1} \psi=\lim _{\tau \rightarrow \infty} \int_{-\tau}^{\tau} \mathfrak{R}_{m}^{j}(\boldsymbol{p}, i y) \psi \frac{\mathrm{d} y}{\pi},
$$

for all $\psi \in \mathscr{H}_{j}$. This yields a convenient representation of its absolute value,

$$
\left|\mathfrak{D}_{m}^{j}\right| \psi=\mathcal{S}_{m}^{j} \mathfrak{D}_{m}^{j} \psi=\lim _{\tau \rightarrow \infty} \int_{-\tau}^{\tau}\left(\mathbb{1}+i y \mathfrak{R}_{m}^{j}(i y)\right) \psi \frac{\mathrm{d} y}{\pi}, \quad \psi \in \operatorname{dom}\left(\mathfrak{D}_{m}^{j}\right),
$$


where, as usual, all arguments $(\boldsymbol{p})$ have been dropped. For convenience we will assume that

$$
H_{\mathrm{f}}^{(m)}-\mathfrak{e} \varphi\left(h_{m}\right)+\mathfrak{e}^{2} b_{m} \geqslant 0,
$$

which is fulfilled by the Hamiltonians in (2.55). Furthermore, we assume the existence of a constant, $\mathfrak{c}_{0}>0$, such that, for all $0 \leqslant m<j \leqslant \infty$,

$$
\begin{gathered}
\left|\boldsymbol{c}_{j}\right|,\left|b_{j}\right|,\left\|\left(1+\omega^{-1 / 2}\right) \boldsymbol{g}_{j}\right\|,\left\|\left(1+\omega^{-1 / 2}\right) h_{j}\right\| \leqslant \mathfrak{c}_{0}, \\
\left|\boldsymbol{c}_{m}^{j}\right|,\left|b_{m}^{j}\right|,\left\|\boldsymbol{g}_{m}^{j}\right\|,\left\|h_{m}^{j}\right\| \leqslant \mathfrak{c}_{0} \rho_{m},\left\|\omega^{-1 / 2} \boldsymbol{g}_{m}^{j}\right\|,\left\|\omega^{-1 / 2} h_{m}^{j}\right\| \leqslant \mathfrak{c}_{0} \rho_{m}^{1 / 2} .
\end{gathered}
$$

The results of Sect. 3 shall imply that, for given $\kappa, \mathfrak{p}>0$, we find some $\mathfrak{e}_{0}>0$ such that, for all $\mathfrak{e} \in\left(0, \mathfrak{e}_{0}\right]$ and $\boldsymbol{p} \in \mathcal{B}_{\mathfrak{p}}$, the choices $\boldsymbol{g}_{m}=\boldsymbol{G}_{m}+\boldsymbol{F}_{m}, h_{m}=$ $\omega f_{m}, \boldsymbol{c}_{m}:=\widetilde{\boldsymbol{c}}_{m}, b_{m}=\widetilde{b}_{m}$ satisfy the assumptions (2.59) and (2.60). This is a consequence of straightforward estimations based on (2.32) and (2.33); recall $(2.34)$. clutter.

We shall again write $K_{j}, \mathfrak{D}_{j}, \mathcal{S}_{j}, \mathfrak{R}_{j}$ instead of $K_{j}^{j}, \mathfrak{D}_{j}^{j}, \mathcal{S}_{j}^{j}, \mathfrak{R}_{j}^{j}$ to reduce

Relative bounds involving the above operators and a discussion of their self-adjointness can be found in Appendix A.

\section{First Results on the Mass Shell and Spectral Gaps}

\subsection{The Spectrum at the Initial Scale}

In the IAPT one infers estimates on the ground state energy and the spectral gap of an operator like $H_{j+1}$ from estimates on the same quantities corresponding to an operator on a preceding scale (i.e. $H_{j}$ ). It is, therefore, important to ensure existence of a spectral gap and estimates on the ground state energy at the initial scale.

First, we recall that $H_{0}^{j}(\boldsymbol{p})=\left(\left(\boldsymbol{p}-\mathbf{p}_{\mathrm{f}}^{(j)}\right)^{2}+1\right)^{1 / 2}+H_{\mathrm{f}}^{(j)}$. Moreover, recall that $\mathbf{p}_{\mathrm{f}}^{(j)}$ and $H_{\mathrm{f}}^{(j)}$ act in $L_{\mathrm{s}}^{2}\left[\left(\mathcal{A}_{j}\right)^{n}\right]$ simply by multiplication with $\boldsymbol{k}_{1}+\cdots+\boldsymbol{k}_{n}$ and $\left|\boldsymbol{k}_{1}\right|+\cdots+\left|\boldsymbol{k}_{n}\right|$, respectively, and $\mathbf{p}_{\mathrm{f}}^{(j)} \Omega_{j}=\mathbf{0}, H_{\mathrm{f}}^{(j)} \Omega_{j}=0$, where $\Omega_{j}$ is the vacuum vector. In particular, it is an elementary exercise to derive the following lemma. We present its proof only for the convenience of the reader:

Lemma 3.1 (spectrum of $H_{0}^{j}(\boldsymbol{p})$ ). For all $\boldsymbol{p} \in \mathbb{R}^{3}$ and $j \in \mathbb{N}_{0} \cup\{\infty\}$, the following holds:

(i) $E_{0}^{j}(\boldsymbol{p})=\left(\boldsymbol{p}^{2}+1\right)^{1 / 2}$ is a fourfold degenerate eigenvalue of $H_{0}^{j}(\boldsymbol{p})$. The corresponding eigenspace is $\left\{v \otimes \Omega_{j}: v \in \mathbb{C}^{4}\right\}$.

(ii) $\operatorname{gap}_{0}^{j}(\boldsymbol{p}) \geqslant \min \left\{\rho_{j}, 1\right\} /\left(2\left(|\boldsymbol{p}|^{2}+1\right)\right)$.

Proof. (i): It is obvious that $\left(\boldsymbol{p}^{2}+1\right)^{1 / 2}$ is an eigenvalue of $H_{0}^{j}(\boldsymbol{p})$ and that $\mathbb{C}^{4} \otimes \Omega_{j}$ belongs to the corresponding eigenspace. It follows from the arguments below that the latter is actually the whole eigenspace (even in the case $j=\infty$ ) and that $\left(\boldsymbol{p}^{2}+1\right)^{1 / 2}$ is the infimum of the spectrum. 
To prove (ii) we observe that the spectrum of $H_{0}^{j}(\boldsymbol{p})$ restricted to the invariant subspace $\left\{\mathbb{C}^{4} \otimes \Omega_{j}\right\}^{\perp}$ is given by the closure of

$$
\bigcup_{n=1}^{\infty}\left\{\left(\left[\boldsymbol{p}-\sum_{i=1}^{n} \boldsymbol{k}_{i}\right]^{2}+1\right)^{1 / 2}+\sum_{i=1}^{n}\left|\boldsymbol{k}_{i}\right|:\left|\boldsymbol{k}_{i}\right| \geqslant \rho_{j}, i=1, \ldots, n\right\} .
$$

On account of the triangle inequality $\left|\sum_{i=1}^{n} \boldsymbol{k}_{i}\right| \leqslant \sum_{i=1}^{n}\left|\boldsymbol{k}_{i}\right|$ this implies

$$
\begin{aligned}
\inf \operatorname{Spec}\left[H _ { 0 } ^ { j } ( \boldsymbol { p } ) \left\lceil_{\left.\left\{\mathbb{C}^{4} \otimes \Omega_{j}\right\}^{\perp}\right]}\right.\right. & \geqslant \inf _{\boldsymbol{k} \in \mathbb{R}^{3}}\left(\left([\boldsymbol{p}-\boldsymbol{k}]^{2}+1\right)^{1 / 2}+\max \left\{\rho_{j},|\boldsymbol{k}|\right\}\right) \\
& =\inf _{r \geqslant 0}\left(\left([|\boldsymbol{p}|-r]^{2}+1\right)^{1 / 2}+\max \left\{\rho_{j}, r\right\}\right) .
\end{aligned}
$$

Writing $g(r):=\left([|\boldsymbol{p}|-r]^{2}+1\right)^{1 / 2}-\left(\boldsymbol{p}^{2}+1\right)^{1 / 2}+\max \left\{\rho_{j}, r\right\}, r \in[0, \infty)$, we find $g^{\prime}>0$ on $\left[\rho_{j}, \infty\right)$; thus $\min _{r \geqslant \rho_{j}} g(r)=g\left(\rho_{j}\right)$. The possible minima of $g$ on $\left[0, \rho_{j}\right]$ are $g\left(\rho_{j}\right), g(0)=\rho_{j}$, and $g(|\boldsymbol{p}|)$, if $|\boldsymbol{p}| \leqslant \rho_{j}$. Since $\mathbb{R} \ni t \mapsto\left(t^{2}+1\right)^{1 / 2}$ is convex Taylor's formula yields, for all $\boldsymbol{p} \in \mathbb{R}^{3}$,

$$
g\left(\rho_{j}\right) \geqslant \rho_{j}-\frac{|\boldsymbol{p}|}{\left(\boldsymbol{p}^{2}+1\right)^{1 / 2}} \rho_{j}=\frac{\rho_{j}}{\left(\boldsymbol{p}^{2}+1\right)^{1 / 2}\left(\left(\boldsymbol{p}^{2}+1\right)^{1 / 2}+|\boldsymbol{p}|\right)}>\frac{\rho_{j}}{2\left(\boldsymbol{p}^{2}+1\right)} .
$$

In the case $|\boldsymbol{p}| \leqslant \rho_{j}$ we apply the inequality

$$
f(t):=1-\left(t^{2}+1\right)^{1 / 2}+t \geqslant \min \{t, 1\} / 2, \quad t \geqslant 0,
$$

to get $g(|\boldsymbol{p}|)=1-\left(\boldsymbol{p}^{2}+1\right)^{1 / 2}+\rho_{j} \geqslant \min \left\{\rho_{j}, 1\right\} / 2$ (At least for $t \in[0,1],(3.2)$ is clear by Taylor's formula; for $t>1$, then, use $f(t)=t f(1 / t))$.

\subsection{A Priori Bounds on the Mass Shell}

Note that the next lemma is valid even when $E_{j}$ belongs to the essential spectrum of $H_{j}$ and when no ground state exists. In particular, it holds for $E=E_{\infty}$. The bound on the derivative of $E_{k}^{j}$ derived in the following lemma justifies the definitions of $\check{H}_{j}(\boldsymbol{p})$ and $\widetilde{H}_{k}^{j}(\boldsymbol{p})$, at least for almost every $\boldsymbol{p} \in \mathcal{B}_{\mathfrak{p}}$, if $\mathfrak{e}$ is sufficiently small. The bound of Lemma 3.2(3) is a crucial ingredient in the estimation of spectral gaps in the next subsection; it follows from the fact that $E_{j}$ tends to infinity as $|\boldsymbol{p}|$ gets large and on the bound on the derivative mentioned before. Here, it suffices to have absolute continuity of $E_{j}$ along every line in $\mathbb{R}^{3}$. This property is fulfilled in many models and is usually proved without regress to the IAPT; see [15]. However, the fact that the derivative of $E_{j}$ is strictly smaller than one, even for arbitrarily large $\boldsymbol{p}$, is a special property of semi-relativistic models: It is false for the non-relativistic model, at least at scale $j=0$.

Lemma 3.2. (1) There exists $\mathfrak{c}>0$ such that, for all $\mathfrak{e} \in(0,1]$ and $\boldsymbol{p} \in \mathbb{R}^{3}$,

$$
(1-\mathfrak{c} \mathfrak{e}) E_{0}(\boldsymbol{p}) \leqslant E_{j}(\boldsymbol{p}) \leqslant(1+\mathfrak{c} \mathfrak{e}) E_{0}(\boldsymbol{p}) .
$$

(2) There is some $\mathfrak{c}>0$ such that, for all $\mathfrak{e} \in(0,1], j \in \mathbb{N} \cup\{\infty\}$, normalized $\boldsymbol{u} \in \mathbb{R}^{3}$, and $\boldsymbol{p} \in \mathbb{R}^{3}$ for which $\partial_{\boldsymbol{u}} E_{j}(\boldsymbol{p}):=\left.\frac{\mathrm{d}}{\mathrm{d} t} E_{j}(\boldsymbol{p}+t \boldsymbol{u})\right|_{t=0}$ exists,

$$
\left|\partial_{\boldsymbol{u}} E_{j}(\boldsymbol{p})\right| \leqslant 1-\frac{1}{2(1+\mathfrak{c} \mathfrak{e}) E_{0}(\boldsymbol{p})^{2}}+\mathfrak{c} \mathfrak{e} E_{0}(\boldsymbol{p}) .
$$


(3) For all $\mathfrak{p}>0$, we find $\mathfrak{e}_{0}>0$ and $\mathfrak{q} \in(0,1 / 2]$ such that

$$
E_{j}(\boldsymbol{p}+\boldsymbol{h})-E_{j}(\boldsymbol{p}) \geqslant-(1-\mathfrak{q})|\boldsymbol{h}|,
$$

for all $\mathfrak{e} \in\left(0, \mathfrak{e}_{0}\right], \boldsymbol{h} \in \mathbb{R}^{3}, \boldsymbol{p} \in \overline{\mathcal{B}}_{\mathfrak{p}}$, and $j \in \mathbb{N}_{0} \cup\{\infty\}$.

Proof. (1): The form bound (A.7) implies $H_{0}^{j} \leqslant(1+\mathfrak{c} \mathfrak{e}) H_{j}$ and (A.8) implies $H_{j} \leqslant(1+\mathfrak{c} \mathfrak{e}) H_{0}^{j}$. Hence, Part $(1)$ is a consequence of the variational principle and the identity $E_{0}^{j}=E_{0}$.

(2): Let $\phi \in \operatorname{dom}\left(H_{\mathrm{f}}^{(j)}\right)$ and $j \in \mathbb{N} \cup\{\infty\}$. On account of Lemma B.1,

$$
H_{j}(\boldsymbol{p}+\boldsymbol{h}) \phi-H_{j}(\boldsymbol{p}) \phi=\partial_{\boldsymbol{h}} H_{j}(\boldsymbol{p}+\boldsymbol{h}) \phi+|\boldsymbol{h}|^{2} \mathcal{O}(1) \phi, \quad|\boldsymbol{h}| \rightarrow 0,
$$

where the norm of $\mathcal{O}(1) \in \mathcal{B}\left(\mathscr{H}_{j}\right)$ can be bounded uniformly in $\boldsymbol{p} \in \mathbb{R}^{3}$ and in $j$. Note that $H_{0}^{j}(\boldsymbol{p}+\boldsymbol{h})=\left(\boldsymbol{v}^{2}+1\right)^{1 / 2}+H_{\mathrm{f}}^{(j)}$ with $\boldsymbol{v}:=\boldsymbol{p}+\boldsymbol{h}-\mathbf{p}_{\mathrm{f}}^{(j)}$. Since the components of $\boldsymbol{v}$ and $\mathbf{p}_{\mathrm{f}}^{(j)}$ commute strongly we obtain

$$
\begin{aligned}
\left\langle\phi \mid \partial_{\boldsymbol{h}} H_{0}^{j}(\boldsymbol{p}+\boldsymbol{h}) \phi\right\rangle & =\left\langle\phi \mid \boldsymbol{h} \cdot \boldsymbol{v}\left(\boldsymbol{v}^{2}+1\right)^{-1 / 2} \phi\right\rangle \\
& \geqslant-|\boldsymbol{h}|\|\phi\|^{2}+|\boldsymbol{h}|\left\langle\phi \mid\left(1-|\boldsymbol{v}|\left(\boldsymbol{v}^{2}+1\right)^{-1 / 2}\right) \phi\right\rangle \\
& \geqslant-|\boldsymbol{h}|\|\phi\|^{2}+|\boldsymbol{h}|\left\langle\phi \mid\left(\boldsymbol{v}^{2}+1\right)^{-1} \phi\right\rangle / 2 .
\end{aligned}
$$

By the trivial bound $\left(\boldsymbol{v}^{2}+1\right)^{-1} \geqslant\left(H_{0}^{j}(\boldsymbol{p}+\boldsymbol{h})\right)^{-2}$ (between multiplication operators $)$ this yields $\partial_{\boldsymbol{h}} H_{0}^{j}(\boldsymbol{p}+\boldsymbol{h}) \geqslant-|\boldsymbol{h}|+|\boldsymbol{h}|\left(H_{0}^{j}(\boldsymbol{p}+\boldsymbol{h})\right)^{-2} / 2$. Next, we have, pointwise at every value of the total momentum,

$$
\left(H_{0}^{j}\right)^{-2}-\left(H_{j}\right)^{-2}=\left(\left(H_{0}^{j}\right)^{-1}-H_{j}^{-1}\right)\left(H_{0}^{j}\right)^{-1}+H_{j}^{-1}\left(\left(H_{0}^{j}\right)^{-1}-H_{j}^{-1}\right) .
$$

Using Lemma A.3(ii) and $\left\|\left(H_{0}^{j}\right)^{-1}\right\|,\left\|H_{j}^{-1}\right\| \leqslant 1$ we find some $\mathfrak{c}>0$ such that

$$
\left\|\left(H_{0}^{j}\right)^{-2}-\left(H_{j}\right)^{-2}\right\| \leqslant 2\left\|\left(H_{j}-H_{0}^{j}\right)\left(H_{0}^{j}\right)^{-1}\right\| \leqslant 2 \mathfrak{c} \mathfrak{e},
$$

at every value of the total momentum. We end up with

$$
\partial_{\boldsymbol{h}} H_{0}^{j}(\boldsymbol{p}+\boldsymbol{h}) \geqslant-|\boldsymbol{h}|+(|\boldsymbol{h}| / 2)\left(H_{j}(\boldsymbol{p}+\boldsymbol{h})\right)^{-2}-\mathfrak{c} \mathfrak{e}|\boldsymbol{h}| .
$$

By virtue of (B.1), $\pm\left\{\partial_{\boldsymbol{h}} H_{j}-\partial_{\boldsymbol{h}} H_{0}^{j}\right\} \leqslant \mathfrak{c} \mathfrak{e}|\boldsymbol{h}|\left(H_{\mathrm{f}}^{(j)}+1\right) \leqslant \mathfrak{c}^{\prime} \mathfrak{e}|\boldsymbol{h}| H_{j}$, whence

$$
\begin{aligned}
H_{j}(\boldsymbol{p}+\boldsymbol{h})-E_{j}(\boldsymbol{p}) \geqslant & H_{j}(\boldsymbol{p}+\boldsymbol{h})-H_{j}(\boldsymbol{p}) \\
\geqslant & -|\boldsymbol{h}|+(|\boldsymbol{h}| / 2) H_{j}(\boldsymbol{p}+\boldsymbol{h})^{-2}-\mathfrak{c} \mathfrak{e}|\boldsymbol{h}| \\
& -\mathfrak{c}^{\prime} \mathfrak{e}|\boldsymbol{h}| H_{j}(\boldsymbol{p}+\boldsymbol{h})-\mathcal{O}\left(|\boldsymbol{h}|^{2}\right) .
\end{aligned}
$$

In particular, we obtain by means of the spectral calculus

$$
\begin{aligned}
E_{j}(\boldsymbol{p}+\boldsymbol{h})-E_{j}(\boldsymbol{p}) \geqslant & -|\boldsymbol{h}|+|\boldsymbol{h}| E_{j}(\boldsymbol{p}+\boldsymbol{h})^{-2} / 2-\mathfrak{c} \mathfrak{e}|\boldsymbol{h}| \\
& -\mathfrak{c}^{\prime} \mathfrak{e}|\boldsymbol{h}| E_{j}(\boldsymbol{p}+\boldsymbol{h})-\mathcal{O}\left(|\boldsymbol{h}|^{2}\right) .
\end{aligned}
$$

Now, let $\boldsymbol{p} \in \mathbb{R}^{3}$, let $\boldsymbol{u} \in \mathbb{R}^{3}$ be normalized, and assume $\partial_{\boldsymbol{u}} E_{j}(\boldsymbol{p})$ exists. Replacing $\boldsymbol{h}$ by $\pm s \boldsymbol{u}$, for small $s>0$, in (3.8), dividing by $\pm s$, and passing to the limit $s \searrow 0$, we obtain an estimate for $\left|\partial_{\boldsymbol{u}} E_{j}(\boldsymbol{p})\right|$ in terms of $E_{j}(\boldsymbol{p})$. Employing (3.3), then, we arrive at (3.4).

(3): We fix $\boldsymbol{p} \in \overline{\mathcal{B}}_{\mathfrak{p}}$ and some normalized $\boldsymbol{u} \in \mathbb{R}^{3}$ and define

$$
e(t):=E_{j}(\boldsymbol{p}+t \boldsymbol{u}), \quad h_{\phi}(t):=\left\langle\phi \mid H_{j}(\boldsymbol{p}+t \boldsymbol{u}) \phi\right\rangle,
$$


for all normalized $\phi \in \operatorname{dom}\left(H_{\mathrm{f}}^{(j)}\right)$ and all $t \in \mathbb{R}$. Thanks to Lemma B.1, we know that $\left|h_{\phi}^{\prime \prime}(t)\right|=\left|\left\langle\phi \mid \partial_{\boldsymbol{u}}^{2} H_{j}(\boldsymbol{p}+t \boldsymbol{u}) \phi\right\rangle\right| \leqslant \mathfrak{c}_{1}$. Consequently, the function defined by $\Delta_{\phi}(t):=-\mathfrak{c}_{1} t^{2}+h_{\phi}(t), t \in \mathbb{R}$, is concave. Thus, $\Delta(t):=\inf \left\{\Delta_{\phi}(t):\right.$ $\left.\phi \in \operatorname{dom}\left(H_{\mathrm{f}}^{(j)}\right),\|\phi\|=1\right\}, t \in \mathbb{R}$, defines a concave function, as well. By a general theorem on concave functions, we know that the left derivative $\Delta_{-}^{\prime}(t)$ and right derivative $\Delta_{+}^{\prime}(t)$ exist, and that $\Delta_{+}^{\prime}$ are both decreasing, and coincide outside a countable set. $\Delta$ is differentiable at every point $t \in \mathbb{R}$ where $\Delta_{-}^{\prime}(t)=$ $\Delta_{+}^{\prime}(t)$. Moreover, $\Delta(t)-\Delta(s)=\int_{s}^{t} \Delta_{ \pm}^{\prime}(r) d r$. Since $e(t)=\Delta(t)+\mathfrak{c}_{1} t^{2}$, the function $e$ also has left and right derivatives $e_{ \pm}^{\prime}$ on $\mathbb{R}$ which coincide almost everywhere, and

$$
e(t)-e(s)=\int_{s}^{t} e_{ \pm}^{\prime}(r) d r, \quad s, t \in \mathbb{R} .
$$

Given $\boldsymbol{h} \neq 0$ we may choose $\boldsymbol{u}:=\boldsymbol{h} /|\boldsymbol{h}|$ and, inserting $t=|\boldsymbol{h}|$ and $s:=0$ into (3.9), we obtain

$$
E_{j}(\boldsymbol{p}+\boldsymbol{h})-E_{j}(\boldsymbol{p})=e(|\boldsymbol{h}|)-e(0)=\int_{0}^{|\boldsymbol{h}|} e^{\prime}(r) d r .
$$

Finally, we prove (3.5). If (3.5) happens to be true, for all $\boldsymbol{p} \in \overline{\mathcal{B}}_{\mathfrak{p}}, \boldsymbol{h} \in \mathbb{R}^{3}, \mathfrak{e} \in$ $(0,1]$, and $\mathfrak{q}=1 / 2$, then there is nothing left to show, of course. So, let $\boldsymbol{p} \in$ $\overline{\mathcal{B}}_{\mathfrak{p}}, \boldsymbol{h} \in \mathbb{R}^{3}, \mathfrak{e} \in(0,1]$, and assume that $E_{j}(\boldsymbol{p}+\boldsymbol{h})-E_{j}(\boldsymbol{p})<-|\boldsymbol{h}| / 2$, which together with (3.3), $E_{j} \geqslant 1, E_{0}(\boldsymbol{p})=\sqrt{\boldsymbol{p}^{2}+1} \leqslant|\boldsymbol{p}|+1$, and $|\boldsymbol{p}| \leqslant \mathfrak{p}$ implies $|\boldsymbol{h}| \leqslant 2(1+\mathfrak{c} \mathfrak{e}) E_{0}(\boldsymbol{p})-2 \leqslant 2 \mathfrak{p}+1$, if $\mathfrak{e}>0$ is sufficiently small, say $\mathfrak{e} \in$ $\left(0, \mathfrak{e}_{0}\right]$. Thus, $E_{0}(\boldsymbol{q}) \leqslant \mathfrak{c}(\mathfrak{p}+1)$, for every $\boldsymbol{q}$ on the line segment from $\boldsymbol{p}$ to $\boldsymbol{p}+\boldsymbol{h}$ we integrate over in (3.10) and for $\mathfrak{e} \in\left(0, \mathfrak{e}_{0}\right]$. Thanks to (3.4) we may conclude that $\left|\partial_{\boldsymbol{u}} E_{j}(\boldsymbol{q})\right| \leqslant 1+\mathfrak{c}^{\prime} \mathfrak{e}(\mathfrak{p}+1)-1 / 4 \mathfrak{c}^{2}(\mathfrak{p}+1)^{2}$, for all $\boldsymbol{q}$ on the same segment, where $\partial_{\boldsymbol{u}} E_{j}(\boldsymbol{q})$ exists, and for small $\mathfrak{e}$. Combining this with (3.10) and decreasing the value of $\mathfrak{e}_{0}$, if necessary, we see that the assertion of (3) holds true.

\subsection{The Spectral Gap}

The results of this subsection shall be applied to the families of operators

$$
\left\{H_{m}^{j}(\boldsymbol{p})\right\}_{m \leqslant j} \quad \text { and } \quad\left\{\widetilde{H}_{m}^{j}(\boldsymbol{p})\right\}_{m \leqslant j},
$$

for $\boldsymbol{p} \in \mathcal{B}_{\mathfrak{p}}$ and for $\mathfrak{e}>0$ at least so small, that Lemma 3.2(3) holds true, for some $\mathfrak{q} \in(0,1)$. In this case $\widetilde{H}_{m}^{j}$ is well defined by Lemma 3.2 almost everywhere on $\mathcal{B}_{\mathfrak{p}}$ to start with. Notice, however, that Theorem 3.5 below together with the type A analyticity of the Hamiltonians $H_{m}^{j}$ proven in Appendix B shows that $E_{j}, j \in \mathbb{N}$, is actually analytic on $\mathcal{B}_{\mathfrak{p}}$, and we may then conclude that $\widetilde{H}_{m}^{j}$ is well defined on all of $\mathcal{B}_{\mathfrak{p}}$.

Recall that, in the case $m<\infty$, the operators $\widetilde{H}_{m}^{j}$ and $H_{m}^{j}$ are unitarily equivalent. This is not true anymore if $m=j=\infty$. Hence, the statements of Lemma 3.4 below involving $\widetilde{H}=\widetilde{H}_{-\infty}^{\infty}$ do not follow at once from 
corresponding statements on $H=H_{-\infty}^{\infty}$. Therefore, we employ the generalized notation of Sect. 2.7. More specifically, we fix the convention

$$
K_{m}^{j} \equiv K_{m}^{j}(\boldsymbol{p}) \text { is either } H_{m}^{j} \text { or } \widetilde{H}_{m}^{j} \text { throughout Sect. 3.3. }
$$

In particular, we then know that

$$
E_{m}^{j}:=\inf \operatorname{Spec}\left(H_{m}^{j}\right)=\inf \operatorname{Spec}\left(\widetilde{H}_{m}^{j}\right)=\inf \operatorname{Spec}\left(K_{m}^{j}\right), \quad m \leqslant j \leqslant \infty,
$$

by unitary equivalence, if $m<\infty$, and by Lemma A.3(iii), if $m=\infty$. Likewise, under the convention (3.11) the spectral gap of $H_{m}^{j}$ defined in (2.23) satisfies

$$
\operatorname{gap}_{m}^{j}=\inf \left(\operatorname{Spec}\left[K_{m}^{j}(\boldsymbol{p})-E_{m}^{j}(\boldsymbol{p})\right] \backslash\{0\}\right), \quad \text { if } m<\infty .
$$

Recall that $K_{m}:=K_{m}^{m}, E_{m}:=E_{m}^{m}, \operatorname{gap}_{m}:=\operatorname{gap}_{m}^{m}$, etc.

The main result of this subsection (Theorem 3.5), an estimate on the spectral gaps of $K_{j}$ and $K_{j}^{j+1}$, is obtained by induction on $j$. In fact, as usual in the IAPT we successively estimate the gaps of

$$
K_{0}^{1}, K_{1}, K_{1}^{2}, \ldots, K_{j}, K_{j}^{j+1}, K_{j+1}, \ldots
$$

Notice that $K_{0}^{1}=H_{0}^{1}$, so that the result of Lemma 3.1 is applicable.

To our knowledge this is the first version of the IAPT that is elaborated for an operator with spin where one expects a degeneracy of eigenvalues. The additional problem that occurs is to rule out a splitting of the lowest eigenvalue which would probably destroy the gap estimate. The absence of a splitting follows, however, from Kramer's degeneracy rule as observed in [28] for (a slight modification of) our model. We also remark that in [28] the authors show the existence of a spectral gap, for strictly positive photon masses and small $\mathfrak{e}>0$, when a pre-factor $\gamma \in(0,1)$ is introduced in front of the squareroot in (1.4) (motivated by requirements of adiabatic perturbation theory).

Lemma 3.3. Let $m, j \in \mathbb{N}_{0} \cup\{\infty\}, m<j$. Assume there exist $\mathfrak{p}, \mathfrak{e}_{0}>0$ such that, for all $\boldsymbol{p} \in \overline{\mathcal{B}}_{\mathfrak{p}}$ and $\mathfrak{e} \in\left(0, \mathfrak{e}_{0}\right], E_{m}(\boldsymbol{p})$ is a fourfold degenerate eigenvalue of $K_{m}(\boldsymbol{p})$. Let $\mathfrak{P}_{m}:=\mathbb{1}_{\left\{E_{m}\right\}}\left(K_{m}\right)$ denote the spectral projection onto the corresponding eigenspace. Then the following holds, at every $\boldsymbol{p} \in \overline{\mathcal{B}}_{\mathfrak{p}}$ and for all $\mathfrak{e} \in\left(0, \mathfrak{e}_{0}\right]:$

(1) $E_{m}$ is the minimum of the spectrum of $K_{m}^{j}$, and $\mathfrak{P}_{m}^{j}:=\mathbb{1}_{\left\{E_{m}\right\}}\left(K_{m}^{j}\right)=$ $\mathfrak{P}_{m} \otimes P_{\Omega_{m}^{j}}$. In particular, the ground state energy of $K_{m}^{j}$ is an exactly four-fold degenerate eigenvalue.

(2) With $\mathfrak{q}$ as in Lemma 3.2(3) we have, for all $\rho \geqslant 0$ and $\lambda>0$,

$$
\begin{aligned}
\operatorname{gap}_{m}^{j} & \geqslant \min \left\{\operatorname{gap}_{m}, \mathfrak{q} \rho_{j}\right\}, \quad E_{m}^{j}=E_{m}, \\
H_{\mathrm{f}}^{(m, j)}+\rho & \leqslant \max \left\{\mathfrak{q}^{-1}, \lambda^{-1}\right\}\left(K_{m}^{j}-E_{m}+\lambda \rho\right) .
\end{aligned}
$$

Proof. We will give the proof only for $H_{m}^{j}$, but by unitary equivalence the result is also valid for $\widetilde{H}_{m}^{j}$. By the natural identification in (2.27) and by 
virtue of (3.5), we obtain

$$
\begin{aligned}
H_{m}^{j}(\boldsymbol{p}) \uparrow_{\mathscr{X}_{n}^{(m, j)}} & \geqslant \int_{\left(\mathcal{A}_{m}^{j}\right)^{n}}^{\oplus}\left(E_{m}(\boldsymbol{p}-\underline{\boldsymbol{k}})+\left|\boldsymbol{k}_{1}\right|+\cdots+\left|\boldsymbol{k}_{n}\right|\right) \mathrm{d} k_{1} \ldots \mathrm{d} k_{n} \\
& \geqslant E_{m}(\boldsymbol{p})+\mathfrak{q} \int_{\left(\mathcal{A}_{m}^{j}\right)^{n}}^{\oplus}\left(\left|\boldsymbol{k}_{1}\right|+\cdots+\left|\boldsymbol{k}_{n}\right|\right) \mathrm{d} k_{1} \ldots \mathrm{d} k_{n} \\
& =\left(E_{m}(\boldsymbol{p})+\mathfrak{q} H_{\mathrm{f}}^{(m, j)}\right) \uparrow_{\mathscr{X}_{n}^{(m, j)}},
\end{aligned}
$$

for $n \geqslant 1$. Since

$$
H_{m}^{j}(\boldsymbol{p}) \uparrow_{\mathscr{X}_{0}^{(m, j)}}=H_{m}(\boldsymbol{p})
$$

we may easily deduce $E_{m}^{j}(\boldsymbol{p})=E_{m}(\boldsymbol{p}), \mathfrak{P}_{m} \otimes P_{\Omega_{m}^{j}} \leqslant \mathfrak{P}_{m}^{j}$, Estimate (3.12) and Estimate (3.13). On the other hand,

$$
\operatorname{ran} \mathfrak{P}_{m}^{j} \subset \operatorname{ker} H_{\mathrm{f}}^{(m, j)}=\mathscr{X}_{0}^{(m, j)} .
$$

Hence, every ground state eigenvector lies in $\mathscr{X}_{0}^{(m, j)}$ and is hence an element of the range of $\mathfrak{P}_{m} \otimes P_{\Omega_{m}^{j}}$. Notice that $\mathbb{1} \otimes P_{\Omega_{m}^{j}}$ is the projection onto $\mathscr{X}_{0}^{(m, j)}$.

In what follows we shall use the notation

$$
\mu_{5}^{j}(\boldsymbol{p}):=\sup _{L \in \mathscr{G}_{4}\left(\mathscr{H}_{j}\right)} \inf \left\{\left\langle\phi \mid\left(K_{j}(\boldsymbol{p})-E_{j}(\boldsymbol{p})\right) \phi\right\rangle: \phi \in \mathcal{Q}\left(H_{\mathrm{f}}^{(j)}\right),\|\phi\|=1, \phi \perp L\right\},
$$

where $\mathscr{G}_{4}\left(\mathscr{H}_{j}\right)$ is the set of four-dimensional subspaces of $\mathscr{H}_{j}$. According to the minimax principle $\mu_{5}^{j}(\boldsymbol{p})$ is the fifth eigenvalue of $K_{j}(\boldsymbol{p})-E_{j}(\boldsymbol{p})$, counting from below including multiplicities, or the lower bottom of the essential spectrum of $K_{j}(\boldsymbol{p})-E_{j}(\boldsymbol{p})$. This notation shall be useful in a situation where we do not already know whether $\mu_{5}^{j}(\boldsymbol{p})$ be equal to $\operatorname{gap}_{j}(\boldsymbol{p})$.

Lemma 3.4. Let $m, j \in \mathbb{N}_{0} \cup\{\infty\}, m<j$, and suppose that all assumptions of Lemma 3.3 are satisfied with $\mathfrak{e}_{0}$ sufficiently small depending on $\kappa$ and $\mathfrak{p}$. Then the following form bounds hold true on $\mathcal{Q}\left(H_{\mathrm{f}}^{(j)}\right)$, at every $\boldsymbol{p} \in \overline{\mathcal{B}}_{\mathfrak{p}}$ and for all $\mathfrak{e} \in\left(0, \mathfrak{e}_{0}\right]$ and $\lambda>0$, with constants depending only on $\mathfrak{p}$ and $\mathfrak{e}_{0}$ :

$$
\begin{aligned}
\left|\mathfrak{D}_{j}\right| & \leqslant \max \{1, \mathfrak{c} / \lambda\}\left(K_{j}-E_{j}+\lambda\right), \\
\pm\left(K_{j}-K_{m}^{j}\right) & \leqslant \mathfrak{c}^{\prime} \mathfrak{e} \max \{1,1 / \lambda\}\left(K_{m}^{j}-E_{m}+\lambda \rho_{m}\right), \\
\pm\left(K_{j}-K_{m}^{j}\right) & \leqslant \mathfrak{c}^{\prime \prime} \mathfrak{e}\left(K_{j}-E_{m}+\rho_{m}\right) .
\end{aligned}
$$

Furthermore,

$$
\left|E_{j}-E_{m}\right| \leqslant \mathfrak{c e} \rho_{m},
$$

and, if $j<\infty$ and gap $_{m}>0$, then

$$
\mu_{5}^{j} \geqslant(1-\mathfrak{c} \mathfrak{e}) \operatorname{gap}_{m}^{j}-\mathfrak{c} \mathfrak{e} \rho_{m} .
$$


Proof. To start with we observe that (3.3) implies the upper bound, $E_{j}(\boldsymbol{p}) \leqslant$ $\left(1+\mathfrak{c} \mathfrak{e}_{0}\right) \max _{|\boldsymbol{q}| \leqslant \mathfrak{p}} E_{0}(\boldsymbol{q})=: C$, which is uniform in $j \in \mathbb{N} \cup\{\infty\}, \mathfrak{e} \in\left(0, \mathfrak{e}_{0}\right]$, and $|\boldsymbol{p}| \leqslant \mathfrak{p}$. From now on we again drop the reference to $\boldsymbol{p}$ in the notation. Then $\left|\mathfrak{D}_{j}\right| \leqslant K_{j}-E_{j}+C$ (recall (2.59)) which implies (3.14). Combining (A.8) and (3.13) we further obtain

$$
\pm\left(K_{j}-K_{m}^{j}\right) \leqslant \mathfrak{c} \mathfrak{e} \rho_{m}\left|\mathfrak{D}_{m}^{j}\right|+\mathfrak{c} \mathfrak{e}\left(K_{m}^{j}-E_{m}+\rho_{m}\right)
$$

on $\mathcal{Q}\left(H_{\mathrm{f}}^{(j)}\right)$. Adding the term $\mathfrak{c} \mathfrak{e} \rho_{m}\left(H_{\mathrm{f}}^{(j)}-\mathfrak{e} \varphi\left(h_{m}\right)+\mathfrak{e}^{2} b_{m}-E_{m}+C\right) \geqslant 0$ to the RHS of (3.19) and absorbing one factor $\rho_{m} \leqslant \kappa$ in the constant we arrive at (3.15). If, say, $\mathfrak{c}^{\prime} \mathfrak{e} \leqslant 1 / 2$, then (3.16) follows from (3.15) and some trivial manipulations. From (3.15) we further deduce that $E_{j}-E_{m} \leqslant K_{j}-E_{m} \leqslant$ $(1+\mathfrak{c} \mathfrak{e})\left(K_{m}^{j}-E_{m}\right)+\mathfrak{c} \mathfrak{e} \rho_{m}$. By Lemma 3.3 we have $E_{m}=\inf \operatorname{Spec}\left[K_{m}^{j}\right]$ and, hence, the variational principle yields $E_{j}-E_{m} \leqslant \mathfrak{c e} \rho_{m}$. Using this we infer the bound $E_{m}-E_{j} \leqslant \mathfrak{c}^{\prime} \mathfrak{e} \rho_{m}$ from (3.16) in a similar way. Altogether this proves (3.17). Writing $\mathcal{N}_{j}:=\operatorname{ker}\left(K_{m}-E_{m}\right) \otimes \Omega_{m}^{j}$ and $\mathcal{S}_{j}:=\left\{\psi \in \mathcal{Q}\left(H_{\mathrm{f}}^{(j)}\right):\|\psi\|=1\right\}$ and employing (3.15) and (3.17) we finally obtain

$$
\begin{aligned}
\mu_{5}^{j} & \geqslant \inf _{\phi \in \mathcal{N}_{j}^{\perp} \cap \mathcal{S}_{j}}\left\langle\phi \mid\left(K_{j}-E_{j}\right) \phi\right\rangle \\
& \geqslant(1-\mathfrak{c} \mathfrak{e}) \inf _{\phi \in \mathcal{N}_{j}^{\perp} \cap \mathcal{S}_{j}}\left\langle\phi \mid\left(K_{m}^{j}-E_{m}\right) \phi\right\rangle+E_{m}-E_{j}-\mathfrak{c} \mathfrak{e} \rho_{m} \\
& \geqslant(1-\mathfrak{c} \mathfrak{e}) \operatorname{gap}_{m}^{j}-\mathfrak{c}^{\prime} \mathfrak{e} \rho_{m} .
\end{aligned}
$$

In the next theorem we combine the previous lemmas in an induction argument to derive a bound on the spectral gaps of $K_{j}$ and $K_{j}^{j+1}, j \in \mathbb{N}$. Let

$$
\vartheta:=\left(\begin{array}{cc}
\sigma_{2} & 0 \\
0 & -\sigma_{2}
\end{array}\right) \mathcal{C}, \quad X_{1}:=\left(\begin{array}{ll}
\mathbb{1} & 0 \\
0 & 0
\end{array}\right), \quad X_{2}:=\left(\begin{array}{ll}
0 & 0 \\
\mathbb{1} & 0
\end{array}\right),
$$

where $\sigma_{2}$ is the second Pauli matrix and $\mathcal{C}$ denotes complex conjugation.

Theorem 3.5 (spectral gaps of $K_{j}$ and $\left.K_{j}^{j+1}\right)$. There exist $\mathfrak{e}_{0}>0$ and $q \in(0, \mathfrak{q})$ such that, at every $\boldsymbol{p} \in \overline{\mathcal{B}}_{\mathfrak{p}}$ and for all $\mathfrak{e} \in\left(0, \mathfrak{e}_{0}\right]$ and $j \in \mathbb{N}_{0}$, the following holds:

(1) $E_{j}=\inf \operatorname{Spec}\left[K_{j}\right]=\inf \operatorname{Spec}\left[K_{j}^{j+1}\right]$ is a fourfold degenerate eigenvalue of both $K_{j}$ and $K_{j}^{j+1}$ and

$$
\operatorname{gap}_{j} \geqslant q \rho_{j} / 2 \text { and } \operatorname{gap}_{j}^{j+1} \geqslant q \rho_{j} / 2 \text {. }
$$

(2) The operators $\vartheta, X_{1}$, and $X_{2}$ commute with $K_{j}$ and $K_{j}^{j+1}$. If $\psi_{j}^{(1)}$ is a normalized ground state eigenvector of $K_{j}$ satisfying $X_{1} \psi_{j}^{(1)}=\psi_{j}^{(1)}$ (which always exists), then four mutually orthonormal ground state eigenvectors are given by $\psi_{j}^{(1)}$ and

$$
\psi_{j}^{(2)}:=X_{2} \psi_{j}^{(1)}, \quad \psi_{j}^{(3)}:=\vartheta \psi_{j}^{(1)}, \quad \psi_{j}^{(4)}:=X_{2} \vartheta \psi_{j}^{(1)} .
$$

(3) Any vector $\psi_{j} \in \mathscr{H}_{j}$ is a ground state eigenvector of $K_{j}$, if and only if $\psi_{j} \otimes \Omega_{j}^{j+1}$ is a ground state eigenvector of $K_{j}^{j+1}$. 
Proof. (1)\&(3): It suffices to prove the following assertions by induction on $j \in \mathbb{N}_{0}$ :

$$
\mathscr{A}_{j}: \Leftrightarrow E_{j} \text { is a } 4 \text {-fold eigenvalue of } K_{j} \text { and } \operatorname{gap}_{j} \geqslant q \rho_{j} / 2 \text {. }
$$

In fact, if $j \in \mathbb{N}_{0}$ and the assertion $\mathscr{A}_{j}$ holds true, then Lemma 3.3 implies $\operatorname{gap}_{j}^{j+1} \geqslant q \min \left\{\rho_{j} / 2, \rho_{j+1}\right\}=q \rho_{j} / 2$ and all the remaining statements of (1) and (3).

Assertion $\mathscr{A}_{0}$ follows, however, from Lemma 3.1, if $|\boldsymbol{p}| \leqslant \mathfrak{p}$, provided that $q$ is sufficiently small.

Now, assume that $\mathscr{A}_{j}$ holds true, for some $j \in \mathbb{N}_{0}$. Then the bound (3.18) is available as well as our conclusion $\operatorname{gap}_{j}^{j+1} \geqslant q \rho_{j} / 2$. In combination they give

$$
\mu_{5}^{j+1} \geqslant(1-\mathfrak{c} \mathfrak{e}) q \rho_{j} / 2-\mathfrak{c} \mathfrak{e} \rho_{j}=q \rho_{j+1}\left(1-\mathfrak{c}^{\prime} \mathfrak{e}\right) \geqslant q \rho_{j+1} / 2,
$$

for small $\mathfrak{e}>0$. In particular, there is some non-vanishing $\phi_{j+1} \in \operatorname{dom}\left(K_{j+1}\right)$ with $K_{j+1} \phi_{j+1}=E_{j+1} \phi_{j+1}$. Below we apply Kramer's degeneracy theorem to show that $E_{j+1}$ is an at least fourfold degenerate eigenvalue of $K_{j+1}$. After that it also follows that $\operatorname{gap}_{j+1}=\mu_{5}^{j+1} \geqslant q \rho_{j+1} / 2$.

(2): Since $\sigma_{2}=\sigma_{2}^{*}$ has purely imaginary entries we have $\vartheta^{2}=-1$ and

$$
\langle\phi \mid \vartheta \psi\rangle=-\langle\psi \mid \vartheta \phi\rangle, \quad \phi, \psi \in \mathscr{H}_{j} .
$$

Obviously, $\vartheta$ leaves $\operatorname{dom}\left(K_{j}\right)=\operatorname{dom}\left(H_{\mathrm{f}}^{(j)}\right)$ invariant and using $\left\{\sigma_{k}, \sigma_{\ell}\right\}=$ $2 \delta_{k \ell} \mathbb{1}_{2}$ and the fact that $\alpha_{0}, \alpha_{1}$, and $\alpha_{3}$ have real entries it is straightforward to check that $\vartheta \mathfrak{D}_{j}=\mathfrak{D}_{j} \vartheta$ on $\operatorname{dom}\left(H_{\mathrm{f}}^{(j)}\right)$. Since the fiber Dirac operator $\mathfrak{D}_{j}$ is essentially self-adjoint on $\operatorname{dom}\left(H_{\mathrm{f}}^{(j)}\right)$ we deduce that it commutes with $\vartheta$ on $\vartheta \operatorname{dom}\left(\mathfrak{D}_{j}\right)=\operatorname{dom}\left(\mathfrak{D}_{j}\right)$ and its resolvent satisfies $\vartheta \mathfrak{R}_{j}(i y)=\mathfrak{R}_{j}(-i y) \vartheta$ on $\mathscr{H}_{j}$. Using, e.g., (2.58) (and a substitution $y \rightarrow-y$ ) we infer that $\vartheta\left|\mathfrak{D}_{j}\right|=\left|\mathfrak{D}_{j}\right| \vartheta$ on $\operatorname{dom}\left(\mathfrak{D}_{j}\right)$. Altogether it follows that $\vartheta K_{j}=K_{j} \vartheta$ on $\operatorname{dom}\left(K_{j}\right)$ and the same argument can be applied to $K_{j}^{j+1}$. In view of (2.22) it is clear that $X_{1}$ and $X_{2}$ commute with the Hamiltonians.

So, let $\psi_{j}^{(1)}$ be as in the statement of Part (2). Then $\psi_{j}^{(3)}=\vartheta \psi_{j}^{(1)} \in$ $\operatorname{dom}\left(K_{j}\right)$ and $K_{j} \psi_{j}^{(3)}=E_{j} \psi_{j}^{(3)}$. Upon choosing $\phi:=\psi:=\psi_{j}^{(1)}$ in (3.23) we further see that $\psi_{j}^{(3)} \perp \psi_{j}^{(1)}$. Since the lower two components of the four-spinors $\psi_{j}^{(1)}=X_{1} \psi_{j}^{(1)}$ and $\psi_{j}^{(3)}=X_{1} \psi_{j}^{(3)}$ are zero and since $K_{j}$ is block diagonal it follows that $\psi_{j}^{(1)}, \ldots, \psi_{j}^{(4)}$ as in (3.22) are mutually orthonormal ground state eigenvectors of $K_{j}$.

\section{Regularity of the Mass Shell}

\subsection{Preparatory Lemmas}

Recall the definitions of $H_{j}^{j+1}$ and $\Pi_{j}^{j+1}$ in Sect. 2.3 and the definitions of $\check{H}_{j+1}$ and $\check{\Pi}_{j+1}$ in Sect. 2.4. Thanks to Theorem 3.5 we know that, on $\mathcal{B}_{\mathfrak{p}}$ and for small $\mathfrak{e}$, both $\Pi_{j}^{j+1}$ and $\check{\Pi}_{j+1}$ are rank four projections and that the 
ground state eigenvalue $E_{j}$ is separated from the rest of the spectrum by a gap satisfying (3.21).

In the following important lemma we compare the Hamiltonians $\check{H}_{j+1}$ and $H_{j}^{j+1}$ on the range of the projection $\Pi_{j}^{j+1}$. Thanks to $(2.35)$ the error term $A_{j}$ in the next lemma is of order $\mathfrak{e} \rho_{j}^{2}$. If it were of order $\mathfrak{e} \rho_{j}$ only, then the proof of Lemma 4.3 did not work. The term $\mathfrak{e}^{2} \varkappa_{j}$ in the next lemma, with

$$
\varkappa_{j}:=\boldsymbol{c}_{j} \cdot \nabla E_{j}-\left\langle f_{j}^{j+1} \mid \omega f_{j}^{j+1}\right\rangle=\left\langle f_{j}^{j+1} \mid \nabla E_{j} \cdot G_{j}^{j+1}\right\rangle \geqslant 0, \quad \varkappa_{j}=\mathcal{O}\left(\rho_{j}\right),
$$

does not cause any harm to Lemma 4.3 since it is non-negative.

Lemma 4.1. For all $\mathfrak{p}>0$, there exist $\mathfrak{c}, \mathfrak{e}_{0}>0$ such that, for all $\mathfrak{e} \in\left(0, \mathfrak{e}_{0}\right]$ and $j \in \mathbb{N}_{0}$, we find some rank four operator $A_{j} \in \mathcal{B}\left(\mathscr{H}_{j+1}\right)$ with $\left\|A_{j}\right\| \leqslant \mathfrak{c e} \rho_{j}^{2}$ and

$$
\begin{aligned}
& \left(\check{H}_{j+1}-H_{j}^{j+1}+\mathfrak{e}^{2} \varkappa_{j}\right) \Pi_{j}^{j+1} \\
& \quad=\mathfrak{e}\left(\Pi_{j}^{+} \nabla H_{j} \Pi_{j}\right) \otimes\left\{a^{\dagger}\left(\boldsymbol{F}_{j}^{j+1}\right)-\mathfrak{e} \check{\boldsymbol{c}}_{j}\right\} P_{\Omega_{j}^{j+1}}+A_{j} .
\end{aligned}
$$

In order to control the difference of various operators attached to succeeding scales in the IAPT it is necessary to prepare a number of resolvent comparison estimates. These are collected in the following lemma where we employ notation introduced in (2.25) and the paragraph below it, (2.26), and (2.40). The resolvents in (4.1)-(4.3) are well-defined because of (3.5) and Theorem 3.5:

Lemma 4.2. Pick $\mathfrak{p}>0$ and let $\delta_{j}$ be some real-valued function of small $\mathfrak{e}>0$ with $\delta_{j}=\mathcal{O}\left(\mathfrak{e} \rho_{j}\right)$. Then we find $\mathfrak{c}, \mathfrak{e}_{0}>0$ such that the following holds, for all $\boldsymbol{p} \in \overline{\mathcal{B}}_{\mathfrak{p}}, \mathfrak{e} \in\left(0, \mathfrak{e}_{0}\right]$, and $j \in \mathbb{N}_{0}$ :

(1) For all $\psi \in \mathscr{H}_{j}$ and $(\boldsymbol{k}, \lambda) \in \mathcal{A}_{j+1}$,

$$
\left\|\left(H_{j}(\boldsymbol{p}-\boldsymbol{k})-E_{j}(\boldsymbol{p})+|\boldsymbol{k}|\right)^{-1} \Pi_{j}^{\perp}(\boldsymbol{p}) \psi\right\| \leqslant \mathfrak{c}\left\|\mathcal{R}_{j}^{\perp}(\boldsymbol{p}) \psi\right\| .
$$

(2) For all $\psi \in \mathscr{X}_{0}^{(j, j+1)} \oplus \mathscr{X}_{1}^{(j, j+1)} \subset \mathscr{H}_{j+1}, s \in\{1 / 2,1\}$, we have the bounds

$$
\begin{aligned}
& \left\|\check{\mathcal{R}}_{j+1}^{\perp}\left(\delta_{j}\right)^{s}\left(\Pi_{j}^{j+1}\right)^{\perp} \psi-\check{\Pi}_{j+1}^{\perp}\left(\left(\mathcal{R}_{j}^{j+1}\right)^{\perp}\right)^{s} \psi\right\| \\
& \quad \leqslant \mathfrak{c} \mathfrak{e} \rho_{j}^{1-s}\left(\|\psi\|+\left\|\left(\mathcal{R}_{j}^{j+1}\right)^{\perp} \psi\right\|\right), \\
& \left\|\check{\mathcal{R}}_{j+1}^{\perp}\left(\delta_{j}\right)\left(\Pi_{j}^{j+1}\right)^{\perp} \psi\right\| \leqslant(1+\mathfrak{c} \mathfrak{e})\left\|\left(\mathcal{R}_{j}^{j+1}\right)^{\perp} \psi\right\|+\mathfrak{c} \mathfrak{e}\|\psi\| .
\end{aligned}
$$

The proofs of Lemma 4.1 and Lemma 4.2 can be found in Appendix C.

\subsection{Proof of $C^{2}$-regularity}

The main objective of this section is to show that $E_{\infty}$ is twice continuously differentiable and strictly convex on $\mathcal{B}_{\mathfrak{p}}$, at least for small $\mathfrak{e}>0$. The starting point of our analysis are the Hellmann-Feynman type formulas (2.28) for the first and second derivatives of the ground state energy $E_{j}, j \in \mathbb{N}$; see Lemma B.1 for their derivation.

Let $\kappa, \mathfrak{p}>0$ be fixed in the whole section. The first step is to bound the difference of the rank four projections $\check{\Pi}_{j+1}$ and $\Pi_{j}^{j+1}$ in terms of $L_{j}^{(1)}$; compare this with Sect. 2.3. 
Lemma 4.3. We find $\mathfrak{c}, \mathfrak{e}_{0}>0$ such that, for all $\boldsymbol{p} \in \overline{\mathcal{B}}_{\mathfrak{p}}, \mathfrak{e} \in\left(0, \mathfrak{e}_{0}\right]$, and $j \in \mathbb{N}_{0}$, $\left\|\check{\Pi}_{j+1}-\Pi_{j}^{j+1}\right\|_{\mathrm{HS}},\left\|\check{\Pi}_{j+1}^{\perp} \Pi_{j}^{j+1}\right\|_{\mathrm{HS}},\left\|\left(\Pi_{j}^{j+1}\right)^{\perp} \check{\Pi}_{j+1}\right\|_{\mathrm{HS}} \leqslant \mathfrak{c} \mathfrak{e} \rho_{j}\left(L_{j}^{(1)}+1\right)$.

Proof. Step 1. First, we consider $\left\|\check{\Pi}_{j+1}^{+} \Pi_{j}^{j+1}\right\|_{\text {HS }}$. Let $\varkappa_{j} \geqslant 0$ be the constant appearing in Lemma 4.1. By (3.17) and (3.21) we have $\left|E_{j+1}-E_{j}\right| \leqslant \mathfrak{c e} \rho_{j} \leqslant$ $\operatorname{gap}_{j+1} / 2$, for small $\mathfrak{e}>0$, and we conclude that

$$
\left\|\left(\check{H}_{j+1}-E_{j}+\mathfrak{e}^{2} \varkappa_{j}\right)^{-1} \check{\Pi}_{j+1}^{\perp}\right\| \leqslant \mathfrak{c} \rho_{j}^{-1} .
$$

Since $\check{H}_{j+1}$ and $H_{j}^{j+1}$ have the same domain, which contains the range of $\Pi_{j}^{j+1}$, this permits us to write

$$
\check{\Pi}_{j+1}^{\perp} \Pi_{j}^{j+1}=\left(\check{H}_{j+1}-E_{j}+\mathfrak{e}^{2} \varkappa_{j}\right)^{-1} \check{\Pi}_{j+1}^{\perp}\left(\check{H}_{j+1}-H_{j}^{j+1}+\mathfrak{e}^{2} \varkappa_{j}\right) \Pi_{j}^{j+1} .
$$

Applying Lemma 4.1 we obtain

$$
\check{\Pi}_{j+1}^{\perp} \Pi_{j}^{j+1}=\left(\check{H}_{j+1}-E_{j}+\mathfrak{e}^{2} \varkappa_{j}\right)^{-1} \check{\Pi}_{j+1}^{\perp} \Theta_{j}+\mathcal{O}\left(\mathfrak{e} \rho_{j}\right),
$$

where the rank four operator

$$
\Theta_{j}:=\mathfrak{e}\left(\Pi_{j}^{\perp} \nabla H_{j} \Pi_{j}\right) \otimes\left\{a^{\dagger}\left(\boldsymbol{F}_{j}^{j+1}\right)-\mathfrak{e} \check{\boldsymbol{c}}_{j}\right\} P_{\Omega_{j}^{j+1}}
$$

satisfies $\left\|\Theta_{j}\right\|_{\mathrm{HS}} \leqslant \mathfrak{c} \mathfrak{e} \rho_{j}$, due to $\left\|\boldsymbol{F}_{j}^{j+1}\right\|,\left|\check{\boldsymbol{c}}_{j}\right| \leqslant \mathfrak{c} \rho_{j}$, see $(2.34)$, and due to the boundedness of $\nabla H_{j}$ relative to $H_{j}$, see (B.7). Since we have $\operatorname{Ran}\left(\Theta_{j}\right) \subset$ $\operatorname{Ran}\left(\Pi_{j}^{j+1}\right)^{\perp} \cap\left\{\mathscr{X}_{0}^{(j, j+1)} \oplus \mathscr{X}_{1}^{(j, j+1)}\right\}$ we may apply (4.3) (with $\delta_{j}=E_{j+1}-E_{j}$ using $\varkappa_{j} \geqslant 0$ ) to deduce that

$$
\left\|\check{\Pi}_{j+1}^{\perp} \Pi_{j}^{j+1}\right\|_{\mathrm{HS}} \leqslant(1+\mathfrak{c} \mathfrak{e})\left\|\left(\mathcal{R}_{j}^{j+1}\right)^{\perp} \Theta_{j}\right\|_{\mathrm{HS}}+\mathfrak{c} \mathfrak{e} \rho_{j} .
$$

Next, we consider the action of $\left(\mathcal{R}_{j}^{j+1}\right)^{\perp}$ on $\Theta_{j}$ more closely, taking into account that $\left(\Pi_{j}^{j+1}\right)^{\perp}=\Pi_{j}^{\perp} \otimes P_{\Omega_{j}^{j+1}}+\mathbb{1} \otimes P_{\Omega_{j}^{j+1}}^{\perp}$ and, hence,

$$
\begin{aligned}
& \left(\mathcal{R}_{j}^{j+1}\right)^{\perp} \uparrow_{\mathscr{X}_{0}^{(j, j+1)}}=\mathcal{R}_{j}^{\perp}, \\
& \left(\mathcal{R}_{j}^{j+1}\right)^{\perp} \uparrow_{\mathscr{X}_{1}^{(j, j+1)}}=\int_{\mathcal{A}_{j}^{j+1}}^{\oplus}\left(H_{j}(\boldsymbol{p}-\boldsymbol{k})-E_{j}(\boldsymbol{p})+|\boldsymbol{k}|\right)^{-1} \mathrm{~d} k .
\end{aligned}
$$

For the member of $\Theta_{j}$ without creation operator we thus have

$$
\left(\mathcal{R}_{j}^{j+1}\right)^{\perp}\left(\Pi_{j}^{\perp} \nabla H_{j} \Pi_{j} \otimes P_{\Omega_{j}^{j+1}}\right)=\left(\mathcal{R}_{j}^{\perp} \nabla H_{j} \Pi_{j}\right) \otimes P_{\Omega_{j}^{j+1}} .
$$

Applying (4.1) we further deduce that

$$
\left\|\left(\mathcal{R}_{j}^{j+1}\right)^{\perp}\left(\Pi_{j}^{\perp} \nabla H_{j} \Pi_{j}\right) \otimes a^{\dagger}\left(\boldsymbol{F}_{j}^{j+1}\right) P_{\Omega_{j}^{j+1}}\right\|_{\mathrm{HS}} \leqslant \mathfrak{c}\left\|\boldsymbol{F}_{j}^{j+1}\right\|\left\|\mathcal{R}_{j}^{\perp} \nabla H_{j} \Pi_{j}\right\|_{\mathrm{HS}}
$$

by computing the Hilbert-Schmidt norm on the LHS in a basis of the form $\left\{e_{i} \otimes e_{\ell}^{\prime}\right\}$, where $\left\{e_{i}\right\}$ and $\left\{e_{\ell}^{\prime}\right\} \ni \Omega_{j}^{j+1}$ are orthonormal bases of $\mathscr{H}_{j}$ and $\mathscr{F}_{j}^{j+1}$, respectively. Using the boundedness of $\nabla H_{j}$ relative to $H_{j}$, see (B.7), as well as $\left\|\Theta_{j}\right\|_{\mathrm{HS}} \leqslant \mathfrak{c} \mathfrak{e} \rho_{j}$, and $\left\|\boldsymbol{F}_{j}^{j+1}\right\|,\left|\check{\boldsymbol{c}}_{j}\right| \leqslant \mathfrak{c} \rho_{j}$, we obtain, for sufficiently small $\mathfrak{e}$,

$$
\left\|\check{\Pi}_{j+1}^{\perp} \Pi_{j}^{j+1}\right\|_{\mathrm{HS}} \leqslant \mathfrak{c e} \rho_{j}\left(L_{j}^{(1)}+1\right) \leqslant 1 / 2 .
$$


Step 2. Next, we turn to $\left\|\check{\Pi}_{j+1}-\Pi_{j}^{j+1}\right\|_{\mathrm{HS}}$ and $\left\|\left(\Pi_{j}^{j+1}\right)^{\perp} \check{\Pi}_{j+1}\right\|_{\mathrm{HS}}$. Choose any orthonormal basis, $\left\{\chi_{k}\right\}_{k=1}^{\infty}$, of $\mathscr{H}_{j+1}$ with $\check{\Pi}_{j+1} \chi_{k}=\chi_{k}$, for $k=1,2,3,4$, and $\check{\Pi}_{j+1}^{\perp} \chi_{k}=\chi_{k}$, for $k \geqslant 5$. Then

$$
\begin{aligned}
\left\|\check{\Pi}_{j+1}-\Pi_{j}^{j+1}\right\|_{\mathrm{HS}}^{2} & =\sum_{k=1}^{\infty}\left\|\left(\check{\Pi}_{j+1}-\Pi_{j}^{j+1}\right) \chi_{k}\right\|^{2} \\
& =\sum_{k=1}^{4}\left\|\left(\Pi_{j}^{j+1}\right)^{\perp} \check{\Pi}_{j+1} \chi_{k}\right\|^{2}+\sum_{k=5}^{\infty}\left\|\Pi_{j}^{j+1} \check{\Pi}_{j+1}^{\perp} \chi_{k}\right\|^{2} .
\end{aligned}
$$

The last expression on the right side of (4.5) is just

$$
\sum_{k=5}^{\infty}\left\|\Pi_{j}^{j+1} \check{\Pi}_{j+1}^{\perp} \chi_{k}\right\|^{2}=\left\|\Pi_{j}^{j+1} \check{\Pi}_{j+1}^{\perp}\right\|_{\mathrm{HS}}^{2}=\left\|\check{\Pi}_{j+1}^{\perp} \Pi_{j}^{j+1}\right\|_{\mathrm{HS}}^{2} .
$$

Thanks to Theorem 3.5(1) we may pick a normalized $\phi_{1} \in \operatorname{Ran}\left(\Pi_{j}^{j+1}\right)$ satisfying $X_{1} \phi_{1}=\phi_{1}$. By (4.4) we have $\left\|\check{\Pi}_{j+1} \phi_{1}\right\| \geqslant 1 / 2$. Thus, we may define $\chi_{1}:=\check{\Pi}_{j+1} \phi_{1} /\left\|\check{\Pi}_{j+1} \phi_{1}\right\|$. Clearly, $X_{1} \chi_{1}=\chi_{1}$. Since $X_{1}, X_{2}$, and $\vartheta$ commute with $U_{j}$ we may choose $\chi_{2}:=X_{2} \chi_{1}, \chi_{3}:=\vartheta \chi_{1}$, and $\chi_{4}=X_{2} \vartheta \chi_{1}$; see Theorem 3.5(2). Moreover,

$$
\begin{aligned}
\left\|\left(\Pi_{j}^{j+1}\right)^{\perp} \check{\Pi}_{j+1}\right\|_{\mathrm{HS}}^{2} & =\sum_{k=1}^{4}\left\|\left(\Pi_{j}^{j+1}\right)^{\perp} \check{\Pi}_{j+1} \chi_{k}\right\|^{2}=4\left\|\left(\Pi_{j}^{j+1}\right)^{\perp} \check{\Pi}_{j+1} \chi_{1}\right\|^{2} \\
& \leqslant 16\left\|\left(\Pi_{j}^{j+1}\right)^{\perp} \check{\Pi}_{j+1} \Pi_{j}^{j+1} \phi_{1}\right\|^{2} \leqslant 16\left\|\check{\Pi}_{j+1}^{\perp} \Pi_{j}^{j+1}\right\|_{\mathrm{HS}}^{2} .
\end{aligned}
$$

Combining (4.4), (4.5), (4.6), and (4.7) we conclude the proof.

Lemma 4.4. There exist $\mathfrak{c}, \mathfrak{e}_{0}>0$ such that, for all $\boldsymbol{p} \in \overline{\mathcal{B}}_{\mathfrak{p}}, \mathfrak{e} \in\left(0, \mathfrak{e}_{0}\right]$, and $j \in \mathbb{N}_{0}$,

$$
L_{j+1}^{(1)} \leqslant(1+\mathfrak{c} \mathfrak{e}) L_{j}^{(1)}+\mathfrak{c} \mathfrak{e}, \quad\left|L_{j+1}^{(1 / 2)}-L_{j}^{(1 / 2)}\right| \leqslant \mathfrak{c} \mathfrak{e} \rho_{j}^{1 / 2}\left(L_{j}^{(1)}+1\right) .
$$

Proof. Let $s \in\{1 / 2,1\}$ and recall the notation (2.41). We derive a bound on the difference between the two numbers

$$
\begin{aligned}
& a_{s}:=\left\|\left(\check{\mathcal{R}}_{j+1}^{\perp}\right)^{s}\left(U_{j} \nabla H_{j+1} U_{j}^{*}\right) \check{\Pi}_{j+1}\right\|_{\mathrm{HS}}, \\
& a_{s}^{\prime}:=\left\|\left\{\left(\mathcal{R}_{j}^{j+1}\right)^{\perp}\right\}^{s} \nabla H_{j}^{j+1} \Pi_{j}^{j+1}\right\|_{\mathrm{HS}} .
\end{aligned}
$$

Notice that $a_{s}=L_{j+1}^{(s)}$ by unitary invariance of the Hilbert-Schmidt norm, and $a_{s}^{\prime}=L_{j}^{(s)}$ because $\Pi_{j}^{j+1}=\Pi_{j} \otimes P_{\Omega_{j}^{j+1}}$ and $\left(\mathcal{R}_{j}^{j+1}\right)^{\perp}$ and $\nabla H_{j}^{j+1}$ reduce to $\mathcal{R}_{j}^{\perp}$ and $\nabla H_{j}$, respectively, on $\mathscr{X}_{0}^{(j, j+1)}=\mathscr{H}_{j} \otimes \mathbb{C} \Omega_{j}^{j+1}$. To compare these two numbers we successively replace each operator in $a_{s}$ by a corresponding one associated with the preceding scale $j$. More precisely, we estimate

$$
\left|a_{s}-a_{s}^{\prime}\right| \leqslant\left|a_{s}-b_{s}\right|+\left|b_{s}-c_{s}\right|+\left|c_{s}-d_{s}\right|+\left|d_{s}-e_{s}\right|+\left|e_{s}-a_{s}^{\prime}\right|,
$$


with

$$
\begin{aligned}
b_{s} & :=\left\|\left(\check{\mathcal{R}}_{j+1}^{\perp}\right)^{s}\left(U_{j} \nabla H_{j+1} U_{j}^{*}\right) \Pi_{j}^{j+1}\right\|_{\mathrm{HS}}, \\
c_{s} & :=\left\|\left(\check{\mathcal{R}}_{j+1}^{\perp}\right)^{s} \nabla H_{j}^{j+1} \Pi_{j}^{j+1}\right\|_{\mathrm{HS}}, \\
d_{s} & :=\left\|\left(\check{\mathcal{R}}_{j+1}^{\perp}\right)^{s}\left(\Pi_{j}^{j+1}\right)^{\perp} \nabla H_{j}^{j+1} \Pi_{j}^{j+1}\right\|_{\mathrm{HS}}, \\
e_{s} & :=\left\|\check{\Pi}_{j+1}^{\perp}\left\{\left(\mathcal{R}_{j}^{j+1}\right)^{\perp}\right\}^{s} \nabla H_{j}^{j+1} \Pi_{j}^{j+1}\right\|_{\mathrm{HS}}
\end{aligned}
$$

Each of the following five steps deals with one of the absolute values on the RHS of (4.9):

Step 1. First, we replace the projection $\check{\Pi}_{j+1}$ in $a_{s}$ by $\Pi_{j}^{j+1}$,

$$
\left|a_{s}-b_{s}\right| \leqslant\left\|\nabla H_{j+1}\left(\mathcal{R}_{j+1}^{\perp}\right)^{s}\right\|\left\|\Pi_{j}^{j+1}-\check{\Pi}_{j+1}\right\|_{\mathrm{HS}} .
$$

From (B.7) and the spectral calculus we deduce that

$$
\left\|\nabla H_{j+1}\left(\mathcal{R}_{j+1}^{\perp}\right)^{s}\right\| \leqslant\left\|\nabla H_{j+1} H_{j+1}^{-1 / 2}\right\|\left\|\frac{H_{j+1}^{1 / 2} \Pi_{j+1}^{\perp}}{\left(H_{j+1}-E_{j+1}\right)^{s}}\right\| \leqslant \mathfrak{c} \rho_{j}^{-s} .
$$

By Lemma 4.3 we obtain $\left|a_{s}-b_{s}\right| \leqslant \mathfrak{c} \mathfrak{e} \rho_{j}^{1-s}\left(L_{j}^{(1)}+1\right)$.

Step 2. Next, we replace the velocity $U_{j} \nabla H_{j+1} U_{j}^{*}$ in $b_{s}$ by $\nabla H_{j}^{j+1}$. This is just a direct application of the bound (B.3) below which together with $\left\|\left(\check{\mathcal{R}}_{j+1}^{\perp}\right)^{s}\right\| \leqslant \mathfrak{c} \rho_{j}^{-s}$ and $\left(H_{\mathrm{f}}^{(j, j+1)}+\rho_{j}\right)^{1 / 2} \Pi_{j}^{j+1}=\rho_{j}^{1 / 2} \Pi_{j}^{j+1}$ implies

$$
\left\|\left(\check{\mathcal{R}}_{j+1}^{\perp}\right)^{s}\left(U_{j} \nabla H_{j+1} U_{j}^{*}-\nabla H_{j}^{j+1}\right) \Pi_{j}^{j+1}\right\|_{\mathrm{HS}} \leqslant \mathfrak{c e} \rho_{j}^{1-s} .
$$

By definition of $b_{s}$ and $c_{s}$ we thus have $\left|b_{s}-c_{s}\right| \leqslant \mathfrak{c e} \rho_{s}^{1-s}$.

Step 3. By (2.28), i.e. $\Pi_{j}^{j+1} \nabla H_{j}^{j+1} \Pi_{j}^{j+1}=\nabla E_{j} \Pi_{j}^{j+1}$, and by Lemma 4.3 we obtain

$$
\left|c_{s}-d_{s}\right| \leqslant\left|\nabla E_{j}\right|\left\|\left(\check{\mathcal{R}}_{j+1}^{\perp}\right)^{s}\right\|\left\|\check{\Pi}_{j+1}^{\perp} \Pi_{j}^{j+1}\right\|_{\mathrm{HS}} \leqslant \mathfrak{c e} \rho_{j}^{1-s}\left(L_{j}^{(1)}+1\right) .
$$

Step 4 . We employ (4.2) with $\delta_{j}:=0$ to deduce that $\left|d_{s}-e_{s}\right| \leqslant \mathfrak{c} \mathfrak{e} \rho_{j}^{1-s}\left(a_{1}^{\prime}+1\right)$, where $a_{1}^{\prime}=L_{j}^{(1)}$.

Step 5. By Lemma 4.3 and the fact that the Hilbert-Schmidt norm dominates the operator norm,

$$
\left|e_{s}-a_{s}^{\prime}\right| \leqslant\left\|\check{\Pi}_{j+1}\left(\Pi_{j}^{j+1}\right)^{\perp}\right\|_{\mathrm{HS}} a_{s}^{\prime} \leqslant \mathfrak{c} \mathfrak{e} \rho_{j}\left(L_{j}^{(1)}+1\right) a_{s}^{\prime} .
$$

Here the projection $\left(\Pi_{j}^{j+1}\right)^{\perp}$ in (4.12) is coming from the resolvent $\left(\mathcal{R}_{j}^{j+1}\right)^{\perp}=$ $\left(\Pi_{j}^{j+1}\right)^{\perp}\left(\mathcal{R}_{j}^{j+1}\right)^{\perp}$ in $e_{s}$ and $a_{s}^{\prime}$.

Collecting the results of the above steps and using $a_{s}=L_{j+1}^{(s)}, a_{s}^{\prime}=L_{j}^{(s)}$, and $a_{s}^{\prime} \leqslant \mathfrak{c} \rho_{j}^{-s}$ (by (B.7)), we arrive at $\left|L_{j+1}^{(s)}-L_{j}^{(s)}\right| \leqslant \mathfrak{c} \mathfrak{e} \rho_{j}^{1-s}\left(L_{j}^{(1)}+1\right)$, which implies (4.8).

Corollary 4.5. There exist $\mathfrak{c}, \mathfrak{e}_{0}>0$ such that, for all $\boldsymbol{p} \in \overline{\mathcal{B}}_{\mathfrak{p}}, \mathfrak{e} \in\left(0, \mathfrak{e}_{0}\right]$, and $j \in \mathbb{N}_{0}$, we have

$$
L_{j}^{(1)} \leqslant(1+\mathfrak{c} \mathfrak{e})^{j}-1, \quad L_{j}^{(1 / 2)} \leqslant \mathfrak{c} \mathfrak{e}\left(1-(1 / 2)^{j / 2}(1+\mathfrak{c} \mathfrak{e})^{j}\right),
$$


as well as

$$
\left\|\check{\Pi}_{j+1}-\Pi_{j}^{j+1}\right\|_{\mathrm{HS}},\left\|\check{\Pi}_{j+1}^{\perp} \Pi_{j}^{j+1}\right\|_{\mathrm{HS}},\left\|\left(\Pi_{j}^{j+1}\right)^{\perp} \check{\Pi}_{j+1}\right\|_{\mathrm{HS}} \leqslant \mathfrak{c} \mathfrak{e}(1+\mathfrak{c} \mathfrak{e})^{j} \rho_{j} .
$$

Proof. First, we prove the bound on $L_{j}^{(1)}$ by means of (4.8) using $L_{0}^{(s)}=$ 0 , which follows from the fact that $\partial_{\boldsymbol{h}} H_{0}$ and $\Pi_{0}$ are merely multiplication operators which commute with each other. After that we use again (4.8) to obtain the recursion formula $L_{j+1}^{(1 / 2)} \leqslant L_{j}^{(1 / 2)}+\mathfrak{c} \mathfrak{e}(1+\mathfrak{c} \mathfrak{e})^{j} \rho_{j}^{1 / 2}$, which together with $L_{0}^{(1 / 2)}=0$ yields the second asserted bound. The remainder of the proof follows from Lemma 4.3 and the bound on $L_{j}^{(1)}$ we have just obtained.

Theorem 4.6. There exist $\mathfrak{c}, \mathfrak{e}_{0}>0$ such that the following assertions hold, for all $\mathfrak{e} \in\left(0, \mathfrak{e}_{0}\right]$ : we have $E_{\infty}=\lim _{j \rightarrow \infty} E_{j}$ in the norm of $C_{b}^{2}\left(\mathcal{B}_{\mathfrak{p}}\right)$ and $\left\|E_{\infty}-E_{0}\right\|_{C_{b}^{2}\left(\mathcal{B}_{\mathfrak{p}}\right)} \leqslant \mathfrak{c} \mathfrak{e}$. More precisely, we have the following estimates:

$$
\begin{aligned}
\left|E_{j}-E_{\infty}\right| & \leqslant \mathfrak{c} \mathfrak{e} \rho_{j}, \\
\left|\partial_{\boldsymbol{h}}\left(E_{j}-E_{\infty}\right)\right| & \leqslant \mathfrak{c} \mathfrak{e}(1+\mathfrak{c} \mathfrak{e})^{j} \rho_{j}, \\
\left|\partial_{\boldsymbol{h}}^{2}\left(E_{j}-E_{\infty}\right)\right| & \leqslant \mathfrak{c} \mathfrak{e}(1+\mathfrak{c} \mathfrak{e})^{j} \rho_{j}^{1 / 2},
\end{aligned}
$$

uniformly on $\mathcal{B}_{\mathfrak{p}}$ and for all $\boldsymbol{h} \in \mathbb{R}^{3},|\boldsymbol{h}|=1$, and $j \in \mathbb{N}_{0}$. In particular, $E_{\infty}$ is strictly convex on $\mathcal{B}_{\mathfrak{p}}$ and attains its global minimum, inf $\mathbb{R}^{3} E_{\infty}$, at $\boldsymbol{p}=\mathbf{0}$.

Proof. For sufficiently small $\mathfrak{e}$, we may infer (4.13) from (3.17). Recall the formulas in (2.28) for the first and second derivative of $E_{m}^{j}=E_{m}$. When we represent $\partial_{\boldsymbol{h}}^{\nu} E_{j+1}, \nu=1,2$, by means of these formulas we actually replace all involved operators by unitarily equivalent ones using the fact that the trace and the Hilbert-Schmidt norm are invariant under conjugation with $U_{j}$. For instance, $4 \partial_{\boldsymbol{h}} E_{j+1}$ is equal to $a_{1}$ with

$$
a_{\nu}:=\operatorname{Tr}\left[\check{\Pi}_{j+1} U_{j}\left\{\partial_{\boldsymbol{h}}^{\nu} H_{j+1}\right\} U_{j}^{*} \check{\Pi}_{j+1}\right], \quad \nu=1,2 .
$$

In order to deal with operators defined on the same Hilbert space we represent $\partial_{\boldsymbol{h}}^{\nu} E_{j}, \nu=1,2$, in terms of the Hamiltonian $H_{j}^{j+1}$. In particular, recalling that $E_{j}=E_{j}^{j+1}$ we write $4 \partial_{\boldsymbol{h}} E_{j}=a_{1}^{\prime}$ with

$$
a_{\nu}^{\prime}:=\operatorname{Tr}\left[\Pi_{j}^{j+1}\left\{\partial_{\boldsymbol{h}}^{\nu} H_{j}^{j+1}\right\} \Pi_{j}^{j+1}\right], \quad \nu=1,2 .
$$

We plan to estimate $\left|a_{\nu}-a_{\nu}^{\prime}\right| \leqslant\left|a_{\nu}-b_{\nu}\right|+\left|b_{\nu}-c_{\nu}\right|+\left|c_{\nu}-a_{\nu}^{\prime}\right|$ with

$$
\begin{aligned}
& b_{\nu}:=\operatorname{Tr}\left[\check{\Pi}_{j+1} U_{j}\left\{\partial_{\boldsymbol{h}}^{\nu} H_{j+1}\right\} U_{j}^{*} \Pi_{j}^{j+1}\right], \\
& c_{\nu}:=\operatorname{Tr}\left[\check{\Pi}_{j+1}\left\{\partial_{\boldsymbol{h}}^{\nu} H_{j}^{j+1}\right\} \Pi_{j}^{j+1}\right],
\end{aligned}
$$

for $\nu=1,2$. Here, we have

$$
\left|a_{\nu}-b_{\nu}\right| \leqslant\left\|\check{\Pi}_{j+1} U_{j}\left\{\partial_{\boldsymbol{h}}^{\nu} H_{j+1}\right\} U_{j}^{*}\right\|_{\mathrm{HS}}\left\|\Pi_{j}^{j+1}-\check{\Pi}_{j+1}\right\|_{\mathrm{HS}},
$$

and because of $\left\|\check{\Pi}_{j+1} U_{j}\left\{\partial_{\boldsymbol{h}}^{\nu} H_{j+1}\right\} U_{j}^{*}\right\|_{\mathrm{HS}}^{2} \leqslant 4\left\|\left\{\partial_{\boldsymbol{h}}^{\nu} H_{j+1}\right\} \Pi_{j+1}\right\|^{2}$ it follows from the boundedness of $\partial_{\boldsymbol{h}}^{\nu} H_{j+1}$ relative to $H_{j+1}$, see (B.7), and Corollary 4.5 
that $\left|a_{\nu}-b_{\nu}\right| \leqslant \mathfrak{c} \mathfrak{e}(1+\mathfrak{c} \mathfrak{e})^{j} \rho_{j}, \nu=1,2$. By (B.3) we further have

$$
\begin{aligned}
\left|b_{\nu}-c_{\nu}\right| \leqslant & \left\|\left(U_{j}\left\{\partial_{\boldsymbol{h}}^{\nu} H_{j+1}\right\} U_{j}^{*}-\partial_{\boldsymbol{h}}^{\nu} H_{j}^{j+1}\right)\left(H_{\mathrm{f}}^{(j, j+1)}+\rho_{j}\right)^{-1 / 2}\right\| \\
& \cdot\left\|\left(H_{\mathrm{f}}^{(j, j+1)}+\rho_{j}\right)^{1 / 2} \Pi_{j}^{j+1}\right\|_{\mathrm{HS}} \cdot\left\|\check{\Pi}_{j+1}\right\|_{\mathrm{HS}} \leqslant \mathfrak{c} \mathfrak{e} \rho_{j},
\end{aligned}
$$

since $\left(H_{\mathrm{f}}^{(j, j+1)}+\rho_{j}\right)^{1 / 2} \Pi_{j}^{j+1}=\rho_{j}^{1 / 2} \Pi_{j}^{j+1}$ by Theorem 3.5(3). Again by (B.7) below and Corollary 4.5 we finally know that

$$
\left|c_{\nu}-a_{\nu}^{\prime}\right| \leqslant\left\|\left\{\partial_{\boldsymbol{h}}^{\nu} H_{j}^{j+1}\right\} \Pi_{j}^{j+1}\right\|_{\mathrm{HS}}\left\|\Pi_{j}^{j+1}-\check{\Pi}_{j+1}\right\|_{\mathrm{HS}} \leqslant \mathfrak{c} \mathfrak{e}(1+\mathfrak{c} \mathfrak{e})^{j} \rho_{j} .
$$

Altogether this yields

$$
4\left|\partial_{\boldsymbol{h}}\left(E_{j}-E_{j+1}\right)\right| \stackrel{\text { if }}{=}=1 a_{\nu}-a_{\nu}^{\prime} \mid \leqslant \mathfrak{c} \mathfrak{e}(1+\mathfrak{c} \mathfrak{e})^{j} \rho_{j}
$$

which implies (4.14), if $\mathfrak{e}$ is sufficiently small; compare (4.20) below.

Next, we turn to the second member in the formula (2.28) for the second derivatives. We have

$$
4 \partial_{\boldsymbol{h}}^{2}\left(E_{j}-E_{j+1}\right)=a_{2}^{\prime}-a_{2}+2 \triangle
$$

with

$$
\triangle:=\left\|\left(\check{\mathcal{R}}_{j+1}^{\perp}\right)^{1 / 2} U_{j}\left\{\partial_{\boldsymbol{h}} H_{j+1}\right\} U_{j}^{*} \check{\Pi}_{j+1}\right\|_{\mathrm{HS}}^{2}-\left\|\left(\left(\mathcal{R}_{j}^{j+1}\right)^{\perp}\right)^{1 / 2}\left\{\partial_{\boldsymbol{h}} H_{j}^{j+1}\right\} \Pi_{j}^{j+1}\right\|_{\mathrm{HS}}^{2} .
$$

Employing the definition (2.44), Lemma 4.4, and Corollary 4.5 we infer that

$$
\begin{aligned}
|\triangle| & =\left|\left(L_{j+1}^{(1 / 2)}+L_{j}^{(1 / 2)}\right)\left(L_{j+1}^{(1 / 2)}-L_{j}^{(1 / 2)}\right)\right| \\
& \leqslant\left(L_{j+1}^{(1 / 2)}+L_{j}^{(1 / 2)}\right)\left|L_{j+1}^{(1 / 2)}-L_{j}^{(1 / 2)}\right| \leqslant \mathfrak{c} \mathfrak{e}^{2}(1+\mathfrak{c} \mathfrak{e})^{j} \rho_{j}^{1 / 2} .
\end{aligned}
$$

Combining (4.17)-(4.19) and using $\rho_{\ell}=\rho_{j}(1 / 2)^{\ell-j}, \ell \geqslant j \geqslant 0$, we get

$$
\sum_{\ell=j}^{\infty}\left|\partial_{\boldsymbol{h}}^{2}\left(E_{\ell+1}-E_{\ell}\right)\right| \leqslant \mathfrak{c} \mathfrak{e} \sum_{\ell=j}^{\infty}(1+\mathfrak{c} \mathfrak{e})^{\ell} \rho_{\ell}^{1 / 2} \leqslant \frac{\mathfrak{c} \mathfrak{e}}{1-b}(1+\mathfrak{c} \mathfrak{e})^{j} \rho_{j}^{1 / 2},
$$

uniformly on $\mathcal{B}_{\mathfrak{p}}$, provided that $\mathfrak{e}>0$ is sufficiently small with $b:=(1+$ $\mathfrak{c} \mathfrak{e})(1 / 2)^{1 / 2}<1$. By the Weierstraß test this implies (4.15). Since $E_{j}$ and $E_{\infty}$ are rotationally symmetric this also implies convergence in $C^{2}\left(\mathcal{B}_{\mathfrak{p}}\right)$.

To discuss the convexity of $E_{\infty}$ we recall that $E_{0}(\boldsymbol{p})=\left(\boldsymbol{p}^{2}+1\right)^{1 / 2}$. Since $\inf _{\mathcal{B}_{\mathfrak{p}}} \partial_{\boldsymbol{p}}^{2} E_{0}>0$, we obtain $\inf _{\mathcal{B}_{\mathfrak{p}}} \partial_{\boldsymbol{p}}^{2} E_{\infty}>0$ from (4.15), provided that $\mathfrak{e}$ is small enough. So $E_{\infty}$ is strictly convex on $\mathcal{B}_{\mathfrak{p}}$. By rotational symmetry, $\nabla E_{\infty}(\mathbf{0})=\mathbf{0}$; thus $E_{\infty}$ attains its unique minimum in $\mathcal{B}_{\mathfrak{p}}$ at $\mathbf{0}$. Thanks to Lemma 3.2(1) we know, however, that for small $\mathfrak{e}$, the global infimum of $E_{\infty}$ is located in $\mathcal{B}_{\mathfrak{p}}$, i.e. at $\boldsymbol{p}=\mathbf{0}$.

\section{Existence and Multiplicity of Ground States}

By Lemma 3.3, Corollary 4.5, and (2.53) we may define the rank four projection

$$
Q(\boldsymbol{p}):=\lim _{j \rightarrow \infty} \widetilde{\Pi}_{j}^{\infty}(\boldsymbol{p})=\lim _{j \rightarrow \infty} \widetilde{\Pi}_{j}(\boldsymbol{p}) \otimes P_{\Omega_{j}^{\infty}},
$$


if $\mathfrak{e}$ is sufficiently small. The goal of this section is to show that $Q$ is the ground state eigenprojection of the operator $\widetilde{H}_{\infty}$, i.e.

$$
Q(\boldsymbol{p})=\widetilde{\Pi}_{\infty}(\boldsymbol{p}):=\mathbb{1}_{\left\{E_{\infty}(\boldsymbol{p})\right\}}\left(\widetilde{H}_{\infty}(\boldsymbol{p})\right) .
$$

This will prove the first assertion in Theorem 1.2(1). From (2.22) and Corollary 4.5 we then also infer that the rate of convergence asserted in Theorem $1.2(1)$ is correct.

The proof is based on the following consequence of (3.16) and (3.17):

$$
\mathfrak{c}_{1}\left(\widetilde{H}_{\infty}-E_{\infty}+\mathfrak{e} \rho_{j}\right) \geqslant \widetilde{H}_{j}^{\infty}-E_{j} .
$$

Proposition 5.1. Let $\boldsymbol{p} \in \mathcal{B}_{\mathfrak{p}}$ and suppose that $\phi$ is a normalized ground state eigenvector of $\widetilde{H}_{\infty}(\boldsymbol{p})$. If $\mathfrak{e}>0$ is sufficiently small depending on $\mathfrak{p}$, then

$$
\liminf _{j \rightarrow \infty}\left\|\widetilde{\Pi}_{j}^{\infty}(\boldsymbol{p}) \phi\right\|>0 .
$$

Proof. Defining $F_{j}(t):=\left(1+t \mathfrak{c}_{1} \mathfrak{e} \rho_{j}\right)^{1 / 2}\left(1+t \mathfrak{c}_{1}\left(\widetilde{H}_{\infty}-E_{\infty}+\mathfrak{e} \rho_{j}\right)\right)^{-1 / 2}, t \geqslant 0, j \in$ $\mathbb{N}$, we observe that, since $\left\|F_{j}\right\| \leqslant 1$,

$$
\begin{aligned}
1 & =\lim _{t \rightarrow \infty}\left\|F_{j}(t) \phi\right\| \leqslant \lim _{t \rightarrow \infty}\left\|F_{j}(t) \mathbb{1} \otimes P_{\Omega_{j}^{\infty}} \phi\right\|+\left\|\mathbb{1} \otimes P_{\Omega_{j}^{\infty}}^{\perp} \phi\right\| \\
& \leqslant \lim _{t \rightarrow \infty}\left\|F_{j}(t) \widetilde{\Pi}_{j}^{\perp} \otimes P_{\Omega_{j}^{\infty}} \phi\right\|+\left\|\widetilde{\Pi}_{j} \otimes P_{\Omega_{j}^{\infty}} \phi\right\|+\left\|\mathbb{1} \otimes P_{\Omega_{j}^{\infty}}^{\perp} \phi\right\|,
\end{aligned}
$$

for every $j \in \mathbb{N}$. If $\mathfrak{e}>0$ is sufficiently small, then (5.3) and the operator monotonicity of the inversion $T \mapsto T^{-1}$ permit to get

$$
\begin{aligned}
\left\|F_{j}(t) \Pi_{j}^{\perp} \otimes P_{\Omega_{j}^{\infty}} \phi\right\|^{2} & \leqslant\left(1+t \mathfrak{c}_{1} \mathfrak{e} \rho_{j}\right)\left\|\left(1+t\left(\widetilde{H}_{j}^{\infty}-E_{j}\right)\right)^{-1 / 2} \widetilde{\Pi}_{j}^{\perp} \otimes P_{\Omega_{j}^{\infty}} \phi\right\|^{2} \\
& =\left(1+t \mathfrak{c}_{1} \mathfrak{e} \rho_{j}\right)\left\|\left\{\left(1+t\left(\widetilde{H}_{j}-E_{j}\right)\right)^{-1 / 2} \widetilde{\Pi}_{j}^{\perp}\right\} \otimes P_{\Omega_{j}^{\infty}} \phi\right\|^{2} \\
& \leqslant \frac{1+t \mathfrak{c}_{1} \mathfrak{e} \rho_{j}}{1+t \operatorname{gap}_{j}} \leqslant \frac{1+t \mathfrak{c}_{1} \mathfrak{e} \rho_{j}}{1+t q \rho_{j} / 2} \stackrel{t \rightarrow \infty}{\longrightarrow} 2 \mathfrak{c}_{1} \mathfrak{e} / q \leqslant \mathfrak{c}_{2}<1,
\end{aligned}
$$

for all $j \in \mathbb{N}$. Since also $\lim _{j \rightarrow \infty}\left\|\left(\mathbb{1} \otimes P_{\Omega_{j}^{\infty}}^{\perp}\right) \phi\right\|=0$ (by dominated convergence) this implies $0<1-\sqrt{\mathfrak{c}_{2}} \leqslant \liminf _{j \rightarrow \infty}\left\|\widetilde{\Pi}_{j}^{\infty} \phi\right\|$.

For later use we record the following observation. In the case $\boldsymbol{p} \neq \mathbf{0}$ we shall use it to produce a contradiction showing that $\phi$ as in the following statement cannot exist:

Corollary 5.2. Given $\mathfrak{p}>0$ we find $\mathfrak{e}_{0}>0$ such that, if $\boldsymbol{p} \in \mathcal{B}_{\mathfrak{p}}, \mathfrak{e} \in\left(0, \mathfrak{e}_{0}\right]$, and if $\phi$ is a normalized ground state eigenvector of $H_{\infty}(\boldsymbol{p})$, then $\left\{\Pi_{j} \otimes P_{\Omega_{j}^{\infty}} \phi\right\}_{j \in \mathbb{N}}$ contains a subsequence with a non-zero weak limit, $\phi^{\prime} \neq 0$.

Proof. The bound (5.3) holds true also with $\widetilde{H}_{\infty}$ and $\widetilde{H}_{j}^{\infty}$ replaced by $H_{\infty}$ and $H_{j}^{\infty}$, respectively. Hence, by exactly the same proof as above (just drop the tildes) we obtain $\liminf _{j \rightarrow \infty}\left\|\Pi_{j} \otimes P_{\Omega_{j}^{\infty}} \phi\right\| \geqslant 1-\sqrt{\mathfrak{c}_{2}}$.

Now, the bounded sequence defined by $\phi_{j}:=\Pi_{j} \otimes P_{\Omega_{j}^{\infty}} \phi, j \in \mathbb{N}$, contains a weakly convergent subsequence, say $\phi_{\varkappa}^{\prime}=\phi_{j_{\varkappa}}, \varkappa \in \mathbb{N}$. Denoting its weak limit by $\phi^{\prime}$, we have $\left\langle\phi \mid \phi^{\prime}\right\rangle=\lim _{\varkappa \rightarrow \infty}\left\langle\phi \mid \phi_{\varkappa}^{\prime}\right\rangle \geqslant\left(1-\sqrt{\mathfrak{c}_{2}}\right)^{2}$, thus $\phi^{\prime} \neq 0$. 
Theorem 5.3 (ground states). For every $\mathfrak{p}>0$, there exists $\mathfrak{e}_{0}>0$ such that, for all $\boldsymbol{p} \in \mathcal{B}_{\mathfrak{p}}$, the ground state energy $E_{\infty}(\boldsymbol{p})$ is an exactly fourfold degenerate eigenvalue of $\widetilde{H}_{\infty}(\boldsymbol{p})$ and the corresponding eigenprojection satisfies

$$
\lim _{j \rightarrow \infty} \widetilde{\Pi}_{j}^{\infty}(\boldsymbol{p})=\widetilde{\Pi}_{\infty}(\boldsymbol{p}) .
$$

In particular, $E_{\infty}(\mathbf{0})$ is a fourfold degenerate eigenvalue of $H_{\infty}(\mathbf{0})$.

Proof. According to Lemma A.3(iii) $\widetilde{H}_{j}^{\infty} \rightarrow \widetilde{H}_{\infty}$ in norm resolvent sense and $E_{\infty}=\lim _{j \rightarrow \infty} E_{j}=\inf \operatorname{Spec}\left[\widetilde{H}_{\infty}\right]$. By the definition of $Q$ in (5.1) this implies $Q=\lim _{j \rightarrow \infty}\left(\widetilde{H}_{j}^{\infty}-E_{j}+1\right)^{-1} \widetilde{\Pi}_{j}^{\infty}=\left(\widetilde{H}_{\infty}-E_{\infty}+1\right)^{-1} Q$, which shows that $\operatorname{Ran}(Q) \subset \operatorname{dom}\left(\widetilde{H}_{\infty}\right)$ and $\widetilde{H}_{\infty} Q=E_{\infty} Q$.

Suppose $\phi$ is some normalized ground state eigenvector of $\widetilde{H}_{\infty}$ contained in the range of $Q^{\perp}$. By Proposition 5.1 and (5.1) we then obtain the contradiction $0=\langle\phi \mid Q \phi\rangle=\lim _{j \rightarrow \infty}\left\|\widetilde{\Pi}_{j}^{\infty} \phi\right\|^{2}>0$. Therefore, $Q=\widetilde{\Pi}_{\infty}$.

\section{Absence of Ground States at Non-Zero Momenta}

While the Hamiltonians $\widetilde{H}_{\infty}(\boldsymbol{p})$ possess ground state eigenvectors, for small $\mathfrak{e}$, the original Hamiltonians $H_{\infty}(\boldsymbol{p})$ do not, unless $\boldsymbol{p}$ is equal to zero. The latter assertion is proved in the present section. To this end we frequently use the abbreviation

$$
\mathscr{R}_{j}(\boldsymbol{p}, \boldsymbol{k}):=\left(H_{j}(\boldsymbol{p}-\boldsymbol{k})-E_{j}(\boldsymbol{p})+|\boldsymbol{k}|\right)^{-1} .
$$

Recall also the abbreviation (2.20) for the resolvent of the fiber Dirac operator and the very last remark in Sect. 2.2. The starting point is the following lemma proved in Appendix D:

Lemma 6.1. Let $\boldsymbol{p} \in \mathbb{R}^{3}, j \in \mathbb{N} \cup\{\infty\}$, and $\mathfrak{e}>0$, and assume that $\phi_{j} \equiv \phi_{j}(\boldsymbol{p})$ is a ground state eigenvector of $H_{j} \equiv H_{j}(\boldsymbol{p})$. Then the following representation is valid, for almost every $k \in \mathcal{A}_{j}$ :

$$
\begin{aligned}
& a(k) \phi_{j}+\mathfrak{e} \boldsymbol{G}_{j}(k) \cdot \mathscr{R}_{j}(\boldsymbol{p}, \boldsymbol{k}) \nabla H_{j} \phi_{j} \\
& =-\mathfrak{e} \int_{\mathbb{R}} \mathscr{R}_{j}(\boldsymbol{p}, \boldsymbol{k}) R_{j}(\boldsymbol{p}-\boldsymbol{k}, i y) \boldsymbol{\alpha} \cdot \boldsymbol{k} R_{j}(\boldsymbol{p}, i y) \boldsymbol{\alpha} \cdot \boldsymbol{G}_{j}(k) R_{j}(\boldsymbol{p}, i y) \phi_{j} \frac{y \mathrm{~d} y}{i \pi} .
\end{aligned}
$$

It immediately implies

Corollary 6.2. Let $\boldsymbol{p} \in \mathbb{R}^{3}, j \in \mathbb{N}_{0}, \mathfrak{e}>0$, and let $\phi_{j}$ be a normalized ground state eigenvector of $H_{j}$. Then, for almost every $k \in \mathcal{A}_{j}$,

$$
\left\|a(k) \phi_{j}+\mathfrak{e} \mathscr{R}_{j}(\boldsymbol{p}, \boldsymbol{k}) \boldsymbol{G}_{j}(k) \cdot \nabla H_{j} \phi_{j}\right\| \leqslant \mathfrak{c} \mathfrak{e} \frac{\mathbb{1}_{\rho_{j} \leqslant|\boldsymbol{k}|<\kappa}}{|\boldsymbol{k}|^{1 / 2}} .
$$

Proof. Follows from $(2.16),|\boldsymbol{k}| \mathscr{R}_{j}(\boldsymbol{p}, \boldsymbol{k})=\mathcal{O}(1)$, and $\left\|R_{j}(i y)\right\| \leqslant\left(1+y^{2}\right)^{-1 / 2}$. 
Together with Corollary 4.5 this implies the following analog of an estimate stated in [10, Proposition 5.1] (with an improved exponent on the RHS, in fact):

Proposition 6.3. Let $\mathfrak{p}>0$. Then there exist $\mathfrak{c}, \mathfrak{e}_{0}>0$ such that, for all $\boldsymbol{p} \in$ $\mathcal{B}_{\mathfrak{p}}, \mathfrak{e} \in\left(0, \mathfrak{e}_{0}\right], j \in \mathbb{N}_{0}$, every normalized ground state eigenvector, $\phi_{j}$, of $H_{j}$, and almost every $k \in \mathcal{A}_{j}$,

$$
\left\|a(k) \phi_{j}+\mathfrak{e} \frac{\boldsymbol{G}_{j}(k) \cdot \nabla E_{j}}{|\boldsymbol{k}|-\boldsymbol{k} \cdot \nabla E_{j}} \phi_{j}\right\| \leqslant \mathfrak{c} \mathfrak{e} \frac{\mathbb{1}_{\rho_{j} \leqslant|\boldsymbol{k}|<\kappa}}{|\boldsymbol{k}|^{1 / 2}} .
$$

Proof. On account of (2.44), (4.1), and Corollary 4.5 we have

$$
\left\|\mathscr{R}_{j}(\boldsymbol{p}, \boldsymbol{k}) \Pi_{j}^{\perp} \nabla H_{j} \phi_{j}\right\| \leqslant \mathfrak{c}\left\|\mathcal{R}_{j}^{\perp} \nabla H_{j} \phi_{j}\right\| \leqslant \mathfrak{c} L_{j}^{(1)} \leqslant \mathfrak{c}^{\prime} .
$$

(Here and below all derivatives are evaluated at $\boldsymbol{p}$.) On account of (6.2) and $\left|\boldsymbol{G}_{j}(k)\right| \leqslant \mathfrak{c} \mathbb{1}_{\rho_{j} \leqslant|\boldsymbol{k}|<\kappa}|\boldsymbol{k}|^{-1 / 2}$ it thus remains to treat $\mathscr{R}_{j}(\boldsymbol{p}, \boldsymbol{k}) \Pi_{j} \nabla H_{j} \phi_{j}=$ $\nabla E_{j} \mathscr{R}_{j}(\boldsymbol{p}, \boldsymbol{k}) \phi_{j}$. For this we again write $\mathcal{R}_{j}(|\boldsymbol{k}|)=\left(H_{j}(\boldsymbol{p})-E_{j}(\boldsymbol{p})+|\boldsymbol{k}|\right)^{-1}$. Then the second resolvent formula implies

$$
\begin{aligned}
\mathscr{R}_{j}(\boldsymbol{p}, \boldsymbol{k}) \phi_{j} & =\mathcal{R}_{j}(|\boldsymbol{k}|) \phi_{j}+\mathscr{R}_{j}(\boldsymbol{p}, \boldsymbol{k})\left\{H_{j}(\boldsymbol{p})-H_{j}(\boldsymbol{p}-\boldsymbol{k})\right\} \mathcal{R}_{j}(|\boldsymbol{k}|) \phi_{j} \\
& =|\boldsymbol{k}|^{-1} \phi_{j}+|\boldsymbol{k}|^{-1} \mathscr{R}_{j}(\boldsymbol{p}, \boldsymbol{k})\left\{\boldsymbol{k} \cdot \nabla H_{j}+\mathcal{O}\left(|\boldsymbol{k}|^{2}\right)\right\} \phi_{j} .
\end{aligned}
$$

Writing $\nabla H_{j} \phi_{j}=\nabla E_{j} \phi_{j}+\Pi_{j}^{\perp} \nabla H_{j} \phi_{j}$ and solving (6.5) for $\mathscr{R}_{j}(\boldsymbol{p}, \boldsymbol{k}) \phi_{j}$ yields

$$
\mathscr{R}_{j}(\boldsymbol{p}, \boldsymbol{k}) \phi_{j}=\frac{1}{|\boldsymbol{k}|-\boldsymbol{k} \cdot \nabla E_{j}}\left(\phi_{j}+\mathscr{R}_{j}(\boldsymbol{p}, \boldsymbol{k})\left\{\Pi_{j}^{\perp} \boldsymbol{k} \cdot \nabla H_{j}+\mathcal{O}\left(|\boldsymbol{k}|^{2}\right)\right\} \phi_{j}\right) .
$$

Here $\mathscr{R}_{j}(\boldsymbol{p}, \boldsymbol{k})\left\{\Pi_{j}^{\perp} \boldsymbol{k} \cdot \nabla H_{j}+\mathcal{O}\left(|\boldsymbol{k}|^{2}\right)\right\} \phi_{j}=\mathcal{O}(|\boldsymbol{k}|)$ by $(6.4)$.

Recall that $\nabla E_{j}(\mathbf{0})=\mathbf{0}$, for all $j \in \mathbb{N}_{0}$, so that the coherent factor in the formula (6.3) vanishes at $\boldsymbol{p}=\mathbf{0}$. As an immediate corollary we observe that the expectation value of the number operator, $N_{\mathrm{f}}$, in a state belonging to the range of $\Pi_{\infty}(\mathbf{0})=\widetilde{\Pi}_{\infty}(\mathbf{0})=\mathbb{1}_{\left\{E_{\infty}(\mathbf{0})\right\}}\left(H_{\infty}(\mathbf{0})\right)$ is finite. Recall also $\widetilde{\Pi}_{j}^{\infty}(\mathbf{0}) \rightarrow \Pi_{\infty}(\mathbf{0})$ in norm, as $j \rightarrow \infty$.

Corollary 6.4. There exist $\mathfrak{c}, \mathfrak{e}_{0}>0$ such that $\operatorname{Ran}\left(\Pi_{\infty}(\mathbf{0})\right) \subset \operatorname{dom}\left(N_{\mathrm{f}}^{1 / 2}\right)$ and $\left\|N_{\mathrm{f}}^{1 / 2} \Pi_{\infty}(\mathbf{0})\right\| \leqslant \mathfrak{c} \mathfrak{e}$, for all $\mathfrak{e} \in\left(0, \mathfrak{e}_{0}\right]$.

Proof. Let $\phi \in \operatorname{dom}\left(N_{\mathrm{f}}^{1 / 2}\right)$ and $\psi \in \mathscr{H}$ both be normalized. Then, by (6.3),

$$
\begin{aligned}
\left|\left\langle N_{\mathrm{f}}^{1 / 2} \phi \mid \Pi_{\infty}(\mathbf{0}) \psi\right\rangle\right|^{2} & \leqslant\left|\lim _{j \rightarrow \infty}\left\langle\phi \mid N_{\mathrm{f}}^{1 / 2} \widetilde{\Pi}_{j}^{\infty}(\mathbf{0}) \psi\right\rangle\right|^{2} \\
& \leqslant \sup _{j \in \mathbb{N}} \int_{\mathbb{R}^{3} \times \mathbb{Z}_{2}}\left\|a(k) \widetilde{\Pi}_{j}^{\infty}(\mathbf{0}) \psi\right\|^{2} \mathrm{~d} k \leqslant \mathfrak{c} \mathfrak{e} \int_{|\boldsymbol{k}|<\kappa} \frac{\mathrm{d}^{3} \boldsymbol{k}}{|\boldsymbol{k}|},
\end{aligned}
$$

which implies the assertion, since $N_{\mathrm{f}}^{1 / 2}$ is self-adjoint.

In an essentially traditional fashion we next infer the absence of ground states of $H_{\infty}(\boldsymbol{p}), \boldsymbol{p} \neq \mathbf{0}$, from Proposition 6.3; compare, e.g., [18,33].

Theorem 6.5. Given $\mathfrak{p}>0$ we find $\mathfrak{e}_{0}>0$ such that, for all $\boldsymbol{p} \in \mathcal{B}_{\mathfrak{p}} \backslash\{\mathbf{0}\}$, and $\mathfrak{e} \in\left(0, \mathfrak{e}_{0}\right]$, the ground state energy $E_{\infty}(\boldsymbol{p})$ is not an eigenvalue of $H_{\infty}(\boldsymbol{p})$. 
Proof. We write $H=H_{\infty}, E=E_{\infty}$. Suppose that $\phi \in \operatorname{dom}(H)$ is normalized and $H(\boldsymbol{p}) \phi=E(\boldsymbol{p}) \phi$. To get a contradiction we exploit that $\nabla E(\boldsymbol{p}) \neq \mathbf{0}$ which follows from the strict convexity of $E$ on $\mathcal{B}_{\mathfrak{p}}$ and $\nabla E(\mathbf{0})=\mathbf{0}$.

Let $\phi_{j}:=\Pi_{j} \otimes P_{\Omega_{j}^{\infty}} \phi$. Borrowing an idea from [18] we pick some $\eta \in$ $\operatorname{dom}\left(N_{\mathrm{f}}^{1 / 2}\right),\|\eta\|=1$. Furthermore, let $g_{r}:=\mathbb{1}_{\{r \leqslant|\boldsymbol{k}|<\kappa\}} \boldsymbol{G} \cdot \nabla E /(|\boldsymbol{k}|-\boldsymbol{k} \cdot \nabla E)$, for $r \in(0, \kappa)$. Then $\left\|g_{r}\right\|^{2}=\mathfrak{c}_{1} \ln (\kappa / r)$ with some $\mathfrak{c}_{1} \in(0, \infty)$ because $0<$ $|\nabla E|<1$. Finally, let $g_{\rho_{j}}^{(j)}:=\mathbb{1}_{\left\{\rho_{j} \leqslant|\boldsymbol{k}|<\kappa\right\}} \boldsymbol{G} \cdot \nabla E_{j} /\left(|\boldsymbol{k}|-\boldsymbol{k} \cdot \nabla E_{j}\right)$. By virtue of (6.3) we then have

$$
\begin{aligned}
& \left\|g_{r}\right\|\left\|\left(N_{\mathrm{f}}+1\right)^{1 / 2} \eta\right\| \\
& \geqslant\left|\left\langle a^{\dagger}\left(g_{r}\right) \eta \mid \phi_{j}\right\rangle\right|=\left|\int_{\mathbb{R}^{3} \times \mathbb{Z}_{2}} g_{r}(k)\left\langle\eta \mid a(k) \phi_{j}\right\rangle \mathrm{d} k\right| \\
& \geqslant \mathfrak{e}\left|\left\langle g_{r} \mid g_{\rho_{j}}^{(j)}\right\rangle\left\langle\eta \mid \phi_{j}\right\rangle\right|-\mathfrak{c} \mathfrak{e} \int_{r \leqslant|\boldsymbol{k}|<\kappa} \frac{|\varepsilon(k) \cdot \nabla E|}{1-\stackrel{k}{\boldsymbol{k}} \cdot \nabla E} \frac{\mathrm{d} k}{|\boldsymbol{k}|^{2}},
\end{aligned}
$$

for every $j \in \mathbb{N}$. By Corollary $5.2\left\{\phi_{j}\right\}_{j \in \mathbb{N}}$ converges weakly to some non-zero vector $\phi^{\prime}$ along some subsequence. We fix $\eta$ such that $\left\langle\eta \mid \phi^{\prime}\right\rangle>0$. Since also $\nabla E_{j} \rightarrow \nabla E$, thus $\left\langle g_{r} \mid g_{\rho_{j}}^{(j)}\right\rangle \rightarrow\left\|g_{r}\right\|^{2}, j \rightarrow \infty$, we arrive at $\mathfrak{c}_{1}^{1 / 2} \ln (\kappa / r)^{1 / 2} \|\left(N_{\mathrm{f}}+\right.$ $1)^{1 / 2} \eta \| \geqslant \mathfrak{e c}_{1} \ln (\kappa / r)\left\langle\eta \mid \phi^{\prime}\right\rangle-\mathfrak{c}^{\prime} \mathfrak{e}(\kappa-r)$. For sufficiently small $r \in(0, \kappa)$, this gives a contradiction.

\section{Coherent Infra-red Representations}

To complete the proofs of our main theorems it only remains to show disjointness of two distinct representations $\pi_{\boldsymbol{p}}$ and $\pi_{\boldsymbol{q}}$, defined consistently in Sect. 2.6 by

$$
\pi_{\boldsymbol{r}}\left(X \otimes \mathbb{1}_{\mathscr{F}_{j}^{\infty}}\right)=W_{j}(\boldsymbol{r}) X W_{j}(\boldsymbol{r})^{*} \otimes \mathbb{1}_{\mathscr{F}_{j}^{\infty}}, \quad X \in \mathcal{B}\left(\mathscr{H}_{j}\right), \quad \boldsymbol{r} \in \mathcal{B}_{\mathfrak{p}} .
$$

This is done in the following corollary. Notice that the strict convexity of $E$ is the crucial ingredient of the proof:

Corollary 7.1. Let $\boldsymbol{p}, \boldsymbol{q} \in \mathcal{B}_{\mathfrak{p}}, \boldsymbol{p} \neq \boldsymbol{q}$. Then the representations $\pi_{\boldsymbol{p}}$ and $\pi_{\boldsymbol{q}}$ of $\mathfrak{A}$ are unitarily inequivalent and, thus, disjoint as they are irreducible.

Proof. Step 1. Let $m \in \mathbb{N}$. We claim that

$$
\lim _{j \rightarrow \infty}\left\langle\Omega_{m}^{j} \mid W_{m}^{j}(\boldsymbol{p}) W_{m}^{j}(\boldsymbol{q})^{*} \Omega_{m}^{j}\right\rangle=0 .
$$

(Recall the notation (2.47)). In fact, we have

$$
\left\langle\Omega_{m}^{j} \mid W_{m}^{j}(\boldsymbol{p}) W_{m}^{j}(\boldsymbol{q})^{*} \Omega_{m}^{j}\right\rangle=\prod_{\ell=m}^{j-1}\left\langle\Omega_{\ell}^{\ell+1} \mid e^{-i \boldsymbol{e} \bar{\omega}\left(h_{\boldsymbol{p}, \boldsymbol{q}}^{(\ell)}\right)} \Omega_{\ell}^{\ell+1}\right\rangle=\prod_{\ell=m}^{j-1} e^{-\frac{\mathrm{e}^{2}}{2}\left\|h_{\boldsymbol{p}, \boldsymbol{q}}^{(\ell)}\right\|^{2}},
$$

where

$$
h_{\boldsymbol{p}, \boldsymbol{q}}^{(\ell)}:=f_{\ell}^{\ell+1}(\boldsymbol{p})-f_{\ell}^{\ell+1}(\boldsymbol{q})=\frac{\boldsymbol{G}_{\ell}^{\ell+1} \cdot \nabla E_{\ell}(\boldsymbol{p})}{\omega-\boldsymbol{k} \cdot \nabla E_{\ell}(\boldsymbol{p})}-\frac{\boldsymbol{G}_{\ell}^{\ell+1} \cdot \nabla E_{\ell}(\boldsymbol{q})}{\omega-\boldsymbol{k} \cdot \nabla E_{\ell}(\boldsymbol{q})} .
$$


By strict convexity of $E$ on $\mathcal{B}_{\mathfrak{p}}$ we have $\left|\nabla E_{\ell}(\boldsymbol{p})-\nabla E_{\ell}(\boldsymbol{q})\right| \geqslant c>0$, for all sufficiently large $\ell \in \mathbb{N}$. Hence, it is elementary to check that $\sum_{\ell=m}^{\infty}\left\|h_{\boldsymbol{p}, \boldsymbol{q}}^{(\ell)}\right\|^{2}=$ $\infty$, which implies (7.1).

Step 2. Suppose for contradiction there exists a unitary $T \in \mathcal{B}(\mathscr{H})$ such that

$$
\pi_{\boldsymbol{p}}(X)=T^{*} \pi_{\boldsymbol{q}}(X) T
$$

for all $X \in \mathfrak{A}$. We consider the expectation of the previous identity in the state $\eta \otimes \Omega$, where $\eta \in \mathbb{C}^{4}$ is normalized and $\Omega$ is the vacuum in $\mathscr{F}$. Choosing

$$
X_{j}=\mathbb{1}_{\mathscr{H}_{m}} \otimes\left\{W_{m}^{j}(\boldsymbol{q})^{*} P_{\Omega_{m}^{j}} W_{m}^{j}(\boldsymbol{q})\right\} \otimes \mathbb{1}_{\mathscr{F}_{j}^{\infty}}
$$

the expectation of both sides in (7.2) then reads

$$
\begin{aligned}
& \left\langle\eta \otimes \Omega \mid \pi_{\boldsymbol{p}}\left(X_{j}\right) \eta \otimes \Omega\right\rangle=\left|\left\langle\Omega_{m}^{j} \mid W_{m}^{j}(\boldsymbol{p}) W_{m}^{j}(\boldsymbol{q})^{*} \Omega_{m}^{j}\right\rangle\right|^{2}, \\
& \left\langle\eta \otimes \Omega \mid T^{*} \pi_{\boldsymbol{q}}\left(X_{j}\right) T \eta \otimes \Omega\right\rangle=\left\langle T(\eta \otimes \Omega) \mid\left(\mathbb{1}_{\mathscr{H}_{m}} \otimes P_{\Omega_{m}^{j}} \otimes \mathbb{1}_{\mathscr{F}_{j}^{\infty}}\right) T(\eta \otimes \Omega)\right\rangle . \\
& \quad \text { According to Step 1 we obtain } \\
& \quad \lim _{j \rightarrow \infty}\left\langle\eta \otimes \Omega \mid \pi_{\boldsymbol{p}}\left(X_{j}\right) \eta \otimes \Omega\right\rangle=0, \\
& \lim _{j \rightarrow \infty}\left\langle\eta \otimes \Omega \mid T^{*} \pi_{\boldsymbol{q}}\left(X_{j}\right) T \eta \otimes \Omega\right\rangle=\left\langle T(\eta \otimes \Omega) \mid\left(\mathbb{1}_{\mathscr{H}_{m}} \otimes P_{\Omega_{m}^{\infty}}\right) T(\eta \otimes \Omega)\right\rangle .
\end{aligned}
$$

Passing to the limit $m \rightarrow \infty$ in the second line of (7.3) we arrive at

$$
\lim _{m \rightarrow \infty}\left\langle T(\eta \otimes \Omega) \mid\left(\mathbb{1}_{\mathscr{H}_{m}} \otimes P_{\Omega_{m}^{\infty}}\right) T(\eta \otimes \Omega)\right\rangle=\|T(\eta \otimes \Omega)\|^{2}=1 .
$$

Under assumption (7.2) we arrive at the contradiction $\|T(\eta \otimes \Omega)\|^{2}=0$.

\section{Acknowledgements}

We thank the Erwin Schrödinger Institute for Mathematical Physics in Vienna for their kind hospitality. O.M. also thanks Li Chen and the Department of Mathematical Sciences at the Tsinghua University in Beijing for their generous hospitality. Parts of this work have been prepared while M.K. was working at the FernUniversität Hagen and while O.M. was working at the Technical University of Clausthal and at the Technical University of Munich. This work has been partially supported by the DFG (SFB/TR12).

\section{Appendix A. Self-Adjointness and Relative Bounds}

\section{A.1. Fiber Dirac Operators}

In this subsection we derive estimates on fiber Dirac operators and we prove their self-adjointness. We employ the notation and general assumptions of Sect. 2.7.

Lemma A.1. $\mathfrak{D}_{m}^{j}(\boldsymbol{p})$ and $\mathfrak{D}_{m}^{j}(\boldsymbol{p})^{2}$ are essentially self-adjoint on $\mathscr{C}_{j}$, for all $\boldsymbol{p} \in \mathbb{R}^{3}$ and $m, j \in \mathbb{N}_{0} \cup\{\infty\}, m \leqslant j$. 
Proof. Assume that $\psi=\left(\psi^{(0)}, \ldots, \psi^{(\ell)}, 0,0, \ldots\right) \in \mathscr{C}_{j}$ and that $\psi^{(n)} \in$ $L_{\mathrm{s}}^{2}\left[\left(\mathbb{R}^{3} \times \mathbb{Z}_{2}\right)^{n}, \mathbb{C}^{4}\right]$ is supported in $\left([-R, R]^{3} \times \mathbb{Z}_{2}\right)^{n}$, for every $n=1, \ldots, \ell$, and some $\ell \in \mathbb{N}$ and $R>\kappa$. A trivial estimate using $\gamma_{m}:=\mathfrak{e} \| \boldsymbol{\alpha} \cdot\left(\varphi\left(\boldsymbol{g}_{m}\right)-\right.$ $\left.\mathfrak{e} \boldsymbol{c}_{m}\right)\left(N_{\mathrm{f}}^{(j)}+1\right)^{-1 / 2} \|<\infty$ shows that

$$
\left\|\mathfrak{D}_{m}^{j}(\boldsymbol{p}) \psi\right\| \leqslant C(m, R, \boldsymbol{p})(\ell+1)\|\psi\|, \quad C(m, R, \boldsymbol{p}):=|\boldsymbol{p}|+R+\gamma_{m} .
$$

Furthermore, we observe that $\left(\mathfrak{D}_{m}^{j}(\boldsymbol{p}) \psi\right)^{(n)}$ is still supported in $([-R$, $\left.R]^{3} \times \mathbb{Z}_{2}\right)^{n}$, for every $n \in \mathbb{N}$, and $\left(\mathfrak{D}_{m}^{j}(\boldsymbol{p}) \psi\right)^{(n)}=0$, for $n=\ell+2, \ell+3, \ldots$. Applying (A.1) repeatedly we conclude, for $\phi \in \mathscr{C}_{j}$, such that $\phi^{(n)}$ is supported in $\left([-R, R]^{3} \times \mathbb{Z}_{2}\right)^{n}$, for every $n \in \mathbb{N}$, and $\phi^{(n)}=0$, for $n=\ell+1, \ell+2, \ldots$,

$$
\left\|\mathfrak{D}_{m}^{j}(\boldsymbol{p})^{\nu s} \phi\right\| \leqslant C(m, R, \boldsymbol{p})^{\nu s} \frac{(\ell+\nu s) !}{\ell !}\|\phi\|, \quad \nu \in\{1,2\} .
$$

Since $\frac{(\ell+\nu s) !}{(\nu s) ! \ell !}=\left(\begin{array}{c}\ell+\nu s \\ \ell\end{array}\right)<2^{\ell+\nu s}$ this implies

$$
\sum_{s=0}^{\infty} \frac{t^{\nu s}}{(\nu s) !}\left\|\mathfrak{D}_{m}^{j}(\boldsymbol{p})^{\nu s} \phi\right\|<\infty, \quad \nu \in\{1,2\},
$$

for all $0<t<2^{-\nu} / C(m, R, \boldsymbol{p})$. Consequently, every $\phi \in \mathscr{C}_{j}$ is an analytic vector for $\mathfrak{D}_{m}^{j}(\boldsymbol{p})$ and a semi-analytic vector for $\mathfrak{D}_{m}^{j}(\boldsymbol{p})^{2}$, which implies the assertion; see [32, Theorems X.39 and X.40].

Lemma A.2. For $\nu>0$, we find $\rho \in(0, \infty)$ and $Y_{\nu}(y), \Upsilon_{\nu} \in \mathscr{L}\left(\mathscr{H}_{j}\right)$ with

$$
\begin{aligned}
\mathfrak{R}_{m}^{j}(i y)\left(H_{\mathrm{f}}^{(j)}+\rho\right)^{-\nu} & =\left(H_{\mathrm{f}}^{(j)}+\rho\right)^{-\nu} \mathfrak{R}_{m}^{j}(i y) Y_{\nu}(y), & & \left\|Y_{\nu}(y)\right\| \leqslant 2, \\
\mathcal{S}_{m}^{j}\left(H_{\mathrm{f}}^{(j)}+\rho\right)^{-\nu} & =\left(H_{\mathrm{f}}^{(j)}+\rho\right)^{-\nu}\left(\mathcal{S}_{m}^{j}+\Upsilon_{\nu}\right), & & \left\|\Upsilon_{\nu}\right\| \leqslant 1,
\end{aligned}
$$

for $y \in \mathbb{R}$. In particular, $\mathfrak{R}_{m}^{j}(i y)$ and $\mathcal{S}_{m}^{j}$ map $\operatorname{dom}\left(\left(H_{\mathrm{f}}^{(j)}\right)^{\nu}\right)$ into itself. Furthermore, the following resolvent identity is valid, for all $\lambda>0$ :

$$
\begin{aligned}
& \left(\mathfrak{R}_{m}^{j}(i y)-\mathfrak{R}_{j}(i y)\right)\left(H_{\mathrm{f}}^{(m, j)}+\lambda\right)^{-1 / 2} \\
& \quad=\mathfrak{R}_{j}(i y) \boldsymbol{\alpha} \cdot\left(\mathfrak{e} \varphi\left(\boldsymbol{g}_{m}^{j}\right)-\mathfrak{e}^{2} c_{m}^{j}\right)\left(H_{\mathrm{f}}^{(m, j)}+\lambda\right)^{-1 / 2} \mathfrak{R}_{m}^{j}(i y) .
\end{aligned}
$$

In particular, the following useful estimate holds true:

$$
\begin{aligned}
& \left|\left\langle\phi \mid\left(\mathfrak{R}_{j}(i y)-\mathfrak{R}_{m}^{j}(i y)\right)\left(H_{\mathrm{f}}^{(m, j)}+\rho_{m}\right)^{-1 / 2} \psi\right\rangle\right| \\
& \quad \leqslant \mathfrak{c} \mathfrak{e} \rho_{m}^{1 / 2}\left\|\mathfrak{R}_{j}(-i y) \phi\right\|\left\|\mathfrak{R}_{m}^{j}(i y) \psi\right\| \leqslant \mathfrak{c} \mathfrak{e} \rho_{m}^{1 / 2}\left(1+y^{2}\right)^{-1},
\end{aligned}
$$

for all normalized $\phi, \psi \in \mathscr{H}_{j}$

Proof. We put $\Theta:=H_{\mathrm{f}}^{(j)}+\rho$ and recall from [26, Lemma 3.2] and [27, Lemma 3.1] that, for every $\nu \in \mathbb{R},\left[\boldsymbol{\alpha} \cdot \varphi\left(\boldsymbol{g}_{m}^{j}\right) \Theta^{-\nu}\right] \Theta^{\nu}$, defined a priori on $\mathscr{C}_{j}$, extends to a bounded operator on $\mathscr{H}_{j}$, henceforth denoted by $T_{\nu}$, and $\left\|T_{\nu}\right\| \leqslant C(\nu, \kappa) / \rho^{1 / 2}$, for all $\nu>0$. We choose $\rho$ so large that $\left\|T_{\nu}\right\| \leqslant 1 / 2$. For $\phi \in \mathscr{C}_{j}$,

$$
\begin{aligned}
& \mathfrak{R}_{m}^{j}(i y) \Theta^{-\nu}\left(\mathfrak{D}_{m}^{j}-i y\right) \phi \\
& \quad=\mathfrak{R}_{m}^{j}(i y)\left(\mathfrak{D}_{m}^{j}-i y\right) \Theta^{-\nu} \phi+\mathfrak{R}_{m}^{j}(i y)\left[\Theta^{-\nu}, \boldsymbol{\alpha} \cdot \varphi\left(\boldsymbol{g}_{m}^{j}\right)\right] \phi \\
& \quad=\left(\mathbb{1}-\mathfrak{R}_{m}^{j}(i y) T_{\nu}\right) \Theta^{-\nu} \mathfrak{R}_{m}^{j}(i y)\left(\mathfrak{D}_{m}^{j}-i y\right) \phi .
\end{aligned}
$$


Since $\mathfrak{D}_{m}^{j}$ is essentially self-adjoint on $\mathscr{C}_{j}$ we know that $\left(\mathfrak{D}_{m}^{j}-i y\right) \mathscr{C}_{j}$ is dense in $\mathscr{H}_{j}$, whence

$$
\mathfrak{R}_{m}^{j}(i y) \Theta^{-\nu}=\left(\mathbb{1}-\mathfrak{R}_{m}^{j}(i y) T_{\nu}\right) \Theta^{-\nu} \mathfrak{R}_{m}^{j}(i y) .
$$

As $\left\|\mathfrak{R}_{m}^{j}(i y) T_{\nu}\right\| \leqslant 1 / 2\left(1+y^{2}\right)^{1 / 2}$ we may define $X_{\nu}(y):=\left(\mathbb{1}-\mathfrak{R}_{m}^{j}(i y) T_{\nu}\right)^{-1}$, so that $X_{\nu}(y) \mathfrak{R}_{m}^{j}(i y) \Theta^{-\nu}=\Theta^{-\nu} \mathfrak{R}_{m}^{j}(i y)$. Thus, we get (A.2) with $Y_{\nu}(y):=$ $X_{\nu}(y)^{*}=\mathbb{1}+Z_{\nu}(y)$, where $\left\|Z_{\nu}(y)\right\| \leqslant 1 /\left(1+y^{2}\right)^{1 / 2}$. Computing the strongly convergent principal value of (A.2) and, using the definition of $\mathcal{S}_{m}^{j}$ in (2.57), we also obtain (A.3). Next, we verify the asserted resolvent identity first on the domain of $H_{\mathrm{f}}^{(j)}$ by applying $\mathfrak{D}_{j}-i y$ on both sides and using that $\mathfrak{R}_{m}^{j}(i y)$ and $\left(H_{\mathrm{f}}^{(m, j)}+\lambda\right)^{-1 / 2}$ commute.

\section{A.2. Bounds on Hamiltonians}

The next lemma applies in particular to the families of operators

$$
\left\{H_{m}^{j}(\boldsymbol{p})\right\}_{m \leqslant j}, \quad\left\{\check{H}_{j}^{j+1}(\boldsymbol{p})\right\}_{j}, \quad \text { and } \quad\left\{\widetilde{H}_{m}^{j}(\boldsymbol{p})\right\}_{m \leqslant j} .
$$

We will use the notation and assumptions for generalized operators introduced in Sect. 2.7.

Lemma A.3. Assume that the estimates in (2.60) hold true. Then there exists $\mathfrak{c}>0$ such that, for all $\boldsymbol{p} \in \mathbb{R}^{3}$ in (2.56), $m, j \in \mathbb{N}_{0} \cup\{\infty\}, m<j, \delta>0$, and $\mathfrak{e} \in(0,1]$, the following holds:

(i) The Hamiltonians $K_{j}$ and $K_{m}^{j}$ are self-adjoint on $\operatorname{dom}\left(H_{\mathrm{f}}^{(j)}\right)$ and essentially self-adjoint on $\mathscr{C}_{j}$.

(ii) For all $\psi \in \operatorname{dom}\left(H_{\mathrm{f}}^{(j)}\right)$,

$$
\begin{aligned}
\left\|\left(K_{j}-K_{m}^{j}\right) \psi\right\| & \leqslant \mathfrak{c} \mathfrak{e} \rho_{m}^{1 / 2}\left\|\left|\mathfrak{D}_{m}^{j}\right|^{1 / 4}\left(H_{\mathrm{f}}^{(m, j)}+\rho_{m}\right)^{1 / 2} \psi\right\| \\
& \leqslant \delta \mathfrak{e} \rho_{m}^{1 / 2}\left\|K_{m}^{j} \psi\right\|+\mathfrak{c} \mathfrak{e} \rho_{m}^{1 / 2}\|\psi\| / \delta^{3}, \\
\left\|\mathfrak{D}_{\ell}^{j} \mid \psi\right\|,\left\|H_{\mathrm{f}}^{(j)} \psi\right\| & \leqslant \mathfrak{c}^{\prime}\left\|K_{m}^{j} \psi\right\|, \quad 0 \leqslant \ell \leqslant j .
\end{aligned}
$$

(iii) $K_{m}^{\infty} \rightarrow K_{\infty}$ in the norm resolvent sense, as $m \rightarrow \infty$.

(iv) The form domain of both $K_{j}$ and $K_{m}^{j}$ is $\mathcal{Q}\left(H_{\mathrm{f}}^{(j)}\right)$ and the following form bounds hold true on $\mathcal{Q}\left(H_{\mathrm{f}}^{(j)}\right)$ :

$$
\begin{aligned}
& \pm\left(K_{j}-K_{m}^{j}\right) \leqslant \delta \mathfrak{e} \rho_{m}\left|\mathfrak{D}_{j}\right|+\mathfrak{c} \mathfrak{e}\left(H_{\mathrm{f}}^{(m, j)}+\rho_{m}\right) / \delta \\
& \pm\left(K_{j}-K_{m}^{j}\right) \leqslant \delta \mathfrak{e} \rho_{m}\left|\mathfrak{D}_{m}^{j}\right|+\mathfrak{c} \mathfrak{e}\left(\delta^{-1}+\delta^{2}\right)\left(H_{\mathrm{f}}^{(m, j)}+\rho_{m}\right) .
\end{aligned}
$$

Proof. First, we derive the relative bounds of (ii) and (iv) on the dense domain $\mathscr{C}_{j}$ : We may write, for $\eta, \psi \in \mathscr{C}_{j}$,

$$
\begin{aligned}
\left\langle\eta \mid\left(K_{j}-K_{m}^{j}\right) \psi\right\rangle= & \mathfrak{e}\left\langle\mathcal{S}_{j} \eta \mid \boldsymbol{\alpha} \cdot\left(\varphi\left(\boldsymbol{g}_{m}^{j}\right)-\mathfrak{e} \boldsymbol{c}_{m}^{j}\right) \psi\right\rangle+\left\langle\eta \mid\left(\mathcal{S}_{j}-\mathcal{S}_{m}^{j}\right) \mathfrak{D}_{m}^{j} \psi\right\rangle \\
& +\mathfrak{e}\left\langle\eta \mid\left(\varphi\left(h_{m}^{j}\right)+\mathfrak{e} b_{m}^{j}\right) \psi\right\rangle \\
= & \mathfrak{e}\left\langle\boldsymbol{\alpha} \cdot\left(\varphi\left(\boldsymbol{g}_{m}^{j}\right)-\mathfrak{e} \boldsymbol{c}_{m}^{j}\right) \eta \mid \mathcal{S}_{m}^{j} \psi\right\rangle+\left\langle\mathfrak{D}_{j} \eta \mid\left(\mathcal{S}_{j}-\mathcal{S}_{m}^{j}\right) \psi\right\rangle \\
& +\mathfrak{e}\left\langle\eta \mid\left(\varphi\left(h_{m}^{j}\right)+\mathfrak{e} b_{m}^{j}\right) \psi\right\rangle .
\end{aligned}
$$

In order to treat the difference of the sign functions let $(r, s)$ be either $(1,0)$ or $(0,1)$, and let $\varepsilon, \varkappa \in(0,1), \varepsilon+\varkappa=1$, and $\eta, \psi \in \mathscr{C}_{j}$. Then a successive 
application of (2.57), (A.4), and $\left\|\left|\mathfrak{D}_{m}^{j}\right|^{\nu} \mathfrak{R}_{m}^{j}(i y)\right\| \leqslant\left(1+y^{2}\right)^{-(1-\nu) / 2}, \nu \in[0,1)$, permits to get

$$
\begin{aligned}
& \left|\left\langle\left(\mathfrak{D}_{j}\right)^{r} \eta \mid\left(\mathcal{S}_{j}-\mathcal{S}_{m}^{j}\right)\left(H_{\mathrm{f}}^{(m, j)}+\rho_{m}\right)^{-1 / 2}\left(\mathfrak{D}_{m}^{j}\right)^{s} \psi\right\rangle\right| \\
& \quad=\left|\lim _{\tau \rightarrow \infty} \int_{-\tau}^{\tau}\left\langle\left(\mathfrak{D}_{j}\right)^{r} \eta \mid\left(\mathfrak{R}_{j}(i y)-\mathfrak{R}_{m}^{j}(i y)\right)\left(H_{\mathrm{f}}^{(m, j)}+\rho_{m}\right)^{-1 / 2}\left(\mathfrak{D}_{m}^{j}\right)^{s} \psi\right\rangle \frac{d y}{\pi}\right| \\
& \quad \leqslant \int_{\mathbb{R}}\left\|\left|\mathfrak{D}_{j}\right|^{r \varkappa} \mathfrak{R}_{j}(-i y)\left|\mathfrak{D}_{j}\right|^{r \varepsilon} \eta\right\| \mathfrak{c} \mathfrak{e} \rho_{m}^{1 / 2}\left\|\left|\mathfrak{D}_{m}^{j}\right|^{s \varkappa} \mathfrak{R}_{m}^{j}(i y)\left|\mathfrak{D}_{m}^{j}\right|^{s \varepsilon} \psi\right\| \frac{d y}{\pi} \\
& \quad \leqslant \mathfrak{c}(\varepsilon) \mathfrak{e} \rho_{m}^{1 / 2}\left\|\left|\mathfrak{D}_{j}\right|^{r \varepsilon} \eta\right\|\left\|\left|\mathfrak{D}_{m}^{j}\right|^{s \varepsilon} \psi\right\| .
\end{aligned}
$$

Choosing $(r, s)=(0,1), \varepsilon:=1 / 4$, and using (2.8), (2.60), $\left|\mathfrak{D}_{m}^{j}\right| \geqslant 1$, and the fact that $\mathfrak{D}_{m}^{j}$ and $\left(H_{\mathrm{f}}^{(m, j)}+\rho_{m}\right)^{-1 / 2}$ commute on $\mathscr{C}_{j}$, we obtain

$$
\begin{aligned}
\left\|\left(K_{j}-K_{m}^{j}\right) \psi\right\|= & \sup _{\|\eta\|=1}\left|\left\langle\eta \mid\left(K_{j}-K_{m}^{j}\right) \psi\right\rangle\right| \\
\leqslant & \mathfrak{e}\left\|\boldsymbol{\alpha} \cdot\left(\varphi\left(\boldsymbol{g}_{m}^{j}\right)-\mathfrak{e} \boldsymbol{c}_{m}^{j}\right) \psi\right\|+\sup _{\|\eta\|=1}\left|\left\langle\eta \mid\left(\mathcal{S}_{j}-\mathcal{S}_{m}^{j}\right) \mathfrak{D}_{m}^{j} \psi\right\rangle\right| \\
& +\mathfrak{e}\left\|\left(\varphi\left(h_{m}^{j}\right)+\mathfrak{e} b_{m}^{j}\right) \psi\right\| \\
\leqslant & \mathfrak{c} \mathfrak{e} \rho_{m}^{1 / 2}\left\|\left|\mathfrak{D}_{m}^{j}\right|^{1 / 4}\left(H_{\mathrm{f}}^{(m, j)}+\rho_{m}\right)^{1 / 2} \psi\right\| \\
\leqslant & \mathfrak{e} \mathfrak{e} \rho_{m}^{1 / 2}\left\|\left|\mathfrak{D}_{m}^{j}\right|^{1 / 2} \psi\right\|^{1 / 2}\left\|\left(H_{\mathrm{f}}^{(m, j)}+\rho_{m}\right) \psi\right\|^{1 / 2} \\
\leqslant & \mathfrak{c} \mathfrak{e} \rho_{m}^{1 / 2}\left\|\left(K_{m}^{j}\right)^{1 / 2} \psi\right\|^{1 / 2}\left\|K_{m}^{j} \psi\right\|^{1 / 2} \\
\leqslant & \mathfrak{c} \mathfrak{e} \rho_{m}^{1 / 2}\|\psi\|^{1 / 4}\left\|K_{m}^{j} \psi\right\|^{3 / 4}
\end{aligned}
$$

In the penultimate step we applied $\left|\mathfrak{D}_{m}^{j}\right| \leqslant K_{m}^{j}$ to the left norm and used that $\left|\mathfrak{D}_{m}^{j}\right|+H_{\mathrm{f}}^{(m)}+\mathfrak{e}\left(\varphi\left(h_{m}\right)+\mathfrak{e} b_{m}\right) \geqslant 0$ and $H_{\mathrm{f}}^{(m, j)}$ commute on $\mathscr{C}_{j}$ and $H_{\mathrm{f}}^{(m)}+H_{\mathrm{f}}^{(m, j)}=H_{\mathrm{f}}^{(j)}$ to bound the right one. By Young's inequality this implies (A.5), for all $\psi \in \mathscr{C}_{j}$. To prove (A.7) we choose $(r, s)=(1,0), \varepsilon:=1 / 2$ in (A.9) and obtain

$$
\begin{aligned}
\left|\left\langle\eta \mid\left(K_{j}-K_{m}^{j}\right) \eta\right\rangle\right| \leqslant & \mathfrak{e}\left\|\boldsymbol{\alpha} \cdot\left(\varphi\left(\boldsymbol{g}_{m}^{j}\right)-\mathfrak{e} \boldsymbol{c}_{m}^{j}\right) \eta\right\|\|\eta\|+\left|\left\langle\mathfrak{D}_{j} \eta \mid\left(\mathcal{S}_{j}-\mathcal{S}_{m}^{j}\right) \eta\right\rangle\right| \\
& +\mathfrak{e}\left\|\left(\varphi\left(h_{m}^{j}\right)+\mathfrak{e} b_{m}^{j}\right) \eta\right\|\|\eta\| \\
\leqslant & \mathfrak{c} \mathfrak{e} \rho_{m}^{1 / 2}\left\|\left|\mathfrak{D}_{j}\right|^{1 / 2} \eta\right\|\left\|\left(H_{\mathrm{f}}^{(m, j)}+\rho_{m}\right)^{1 / 2} \eta\right\|,
\end{aligned}
$$

which holds, for all $\delta>0$ and $\eta \in \mathscr{C}_{j}$. Setting $h_{m}^{j}=0$ and $b_{m}^{j}=0$ for the moment we see that (A.7) with $1 / \delta=2 \mathfrak{e} \rho_{m}$ implies $\left|\mathfrak{D}_{j}\right| \leqslant \mathfrak{c}\left(\left|\mathfrak{D}_{m}^{j}\right|+H_{\mathrm{f}}^{(m, j)}\right)$, from which we finally infer (A.8) (for non-zero $h_{m}^{j}$ and $b_{m}^{j}$ ).

By (A.5) we have

$$
\left\|\left(K_{m}^{j}-K_{0}^{j}\right) \psi\right\| \leqslant \varepsilon\left\|K_{0}^{j} \psi\right\|+\mathfrak{c}\|\psi\|, \quad\left\|K_{m}^{j} \psi\right\| \leqslant \mathfrak{c}^{\prime}\left\|K_{0}^{j} \psi\right\|,
$$

for $\psi \in \mathscr{C}_{j}$. Since $K_{0}^{j}=\left(\left(\boldsymbol{p}-\mathbf{p}_{\mathrm{f}}^{(j)}\right)^{2}+\mathbb{1}\right)^{1 / 2}+H_{\mathrm{f}}^{(j)}$ is obviously self-adjoint on $\operatorname{dom}\left(H_{\mathrm{f}}^{(j)}\right)$ and essentially self-adjoint on $\mathscr{C}_{j}$ this proves Part (i) by virtue of the Kato-Rellich theorem. We also conclude that (A.5) extends to every $\psi \in$ 
$\operatorname{dom}\left(H_{\mathrm{f}}^{(j)}\right)$. Since $\left\|H_{\mathrm{f}}^{(j)} \psi\right\| \leqslant\left\|K_{0}^{j} \psi\right\|$ and $\left\|\left|\mathfrak{D}_{\ell}^{j}\right| \psi\right\| \leqslant \mathfrak{c}^{\prime}\left(\left\|K_{\ell}^{j} \psi\right\|+\left\|H_{\mathrm{f}}^{(j)} \psi\right\|\right)$ we further obtain (A.6).

(iii): Choosing $j=\infty$ we readily infer from (A.5) and the second resolvent identity that $\left\|\left(K_{m}^{\infty}-i\right)^{-1}-\left(K_{\infty}-i\right)^{-1}\right\| \leqslant \mathfrak{c} \mathfrak{e} \rho_{m}^{1 / 2} \rightarrow 0$, as $m \rightarrow \infty$.

Remark A.4. We can use the arguments of the above proof to compare Hamiltonians $H_{m}^{j}$ with the same scale parameters but for different fibers, say $\boldsymbol{p}$ and $\boldsymbol{p}+\boldsymbol{h}$. For instance, choose $K_{m}^{j}=H_{m}^{j}(\boldsymbol{p}), \mathfrak{D}_{m}^{j}=D_{m}^{j}(\boldsymbol{p})$, etc., and replace

$$
\begin{aligned}
\left(K_{j}(\boldsymbol{p}), \mathcal{S}_{j}(\boldsymbol{p}), \mathfrak{D}_{j}(\boldsymbol{p}), \mathfrak{R}_{j}(\boldsymbol{p}, i y), H_{\mathrm{f}}^{(m, j)}+\rho_{m}\right) & \\
& \longmapsto\left(H_{m}^{j}(\boldsymbol{p}+\boldsymbol{h}), S_{m}^{j}(\boldsymbol{p}+\boldsymbol{h}), D_{m}^{j}(\boldsymbol{p}+\boldsymbol{h}), R_{j}(\boldsymbol{p}+\boldsymbol{h}, i y), \mathbb{1}\right) .
\end{aligned}
$$

In accordance, replace $\mathfrak{e} \varphi\left(\boldsymbol{g}_{m}^{j}\right)-\mathfrak{e}^{2} \boldsymbol{c}_{m}^{j}$ by $\boldsymbol{h}$, set $h_{m}^{j}=b_{m}^{j}=0$, and replace $\mathfrak{e} \rho_{j}^{1 / 2}$ by $|\boldsymbol{h}|$. Then we immediately obtain by inspection of the blocks (A.9) and (A.10) of the above proof that

$$
\left\|\left(H_{m}^{j}(\boldsymbol{p}+\boldsymbol{h})-H_{m}^{j}(\boldsymbol{p})\right) \psi\right\| \leqslant \mathfrak{c}|\boldsymbol{h}|\left\|\left|D_{m}^{j}(\boldsymbol{p})\right|^{1 / 4} \psi\right\|,
$$

and thus by monotonicity

$$
\left\|\left(H_{m}^{j}(\boldsymbol{p}+\boldsymbol{h})-H_{m}^{j}(\boldsymbol{p})\right) \psi\right\| \leqslant \mathfrak{c}|\boldsymbol{h}|\left\|H_{m}^{j}(\boldsymbol{p})^{1 / 4} \psi\right\|,
$$

for all $\boldsymbol{p}, \boldsymbol{h} \in \mathbb{R}, m, j \in \mathbb{N}_{0} \cup\{\infty\}, m \leqslant j$, and $\psi \in \operatorname{dom}\left(H_{\mathrm{f}}^{(j)}\right)$.

\section{Appendix B. Analyticity and Hellmann-Feynman Formulas}

Lemma B.1. For all $m, j \in \mathbb{N}_{0}$ and $\mathfrak{e} \in(0,1]$, the following holds true:

(1) $\left\{H_{m}^{j}(\boldsymbol{p})\right\}_{\boldsymbol{p} \in \mathbb{R}^{3}}$ extends to an analytic family of type $A$ indexed by the set $\left\{\boldsymbol{z} \in \mathbb{C}^{3}:|\operatorname{Im} \boldsymbol{z}|<1\right\}$.

(2) For $\boldsymbol{p}, \boldsymbol{h} \in \mathbb{R}^{3}$, we know that $\partial_{\boldsymbol{h}} H_{m}^{j}(\boldsymbol{p})$ is bounded relative to $H_{m}^{j}(\boldsymbol{p})^{1 / 4}$. All higher derivatives of $H_{m}^{j}$ are bounded uniformly in $\boldsymbol{p} \in \mathbb{R}^{3}, m$, and $j$.

(3) For $\boldsymbol{p}, \boldsymbol{h} \in \mathbb{R}^{3}$ and $\ell \in \mathbb{N}$, we have

$$
\left\|\left(\partial_{\boldsymbol{h}}^{\ell} H_{j}-\partial_{\boldsymbol{h}}^{\ell} H_{m}^{j}\right)\left(H_{\mathrm{f}}^{(m, j)}+\rho_{m}\right)^{-1 / 2}\right\| \leqslant \mathfrak{c}(\ell) \mathfrak{e} \rho_{m}^{1 / 2}|\boldsymbol{h}|^{\ell} .
$$

(4) Let $\mathfrak{p}, \mathfrak{e}_{0}>0$ and assume that $E_{m}^{j}$ is an isolated eigenvalue of constant, finite multiplicity of $H_{m}^{j}$ on the ball $\mathcal{B}_{\mathfrak{p}}$, for all $\mathfrak{e} \in\left(0, \mathfrak{e}_{0}\right]$. For all $\boldsymbol{h} \in \mathbb{R}^{3}$, we then have

$$
\begin{aligned}
\partial_{\boldsymbol{h}} E_{m}^{j} & =\operatorname{Tr}\left\{\Pi_{m}^{j}\left(\partial_{\boldsymbol{h}} H_{m}^{j}\right) \Pi_{m}^{j}\right\} / 4, \\
\partial_{\boldsymbol{h}}^{2} E_{m}^{j} & =\operatorname{Tr}\left\{\Pi_{m}^{j}\left(\partial_{\boldsymbol{h}}^{2} H_{m}^{j}\right) \Pi_{m}^{j}\right\} / 4-\left\|\left(\left(\mathcal{R}_{m}^{j}\right)^{\perp}\right)^{1 / 2}\left(\partial_{\boldsymbol{h}} H_{m}^{j}\right) \Pi_{m}^{j}\right\|_{\mathrm{HS}}^{2} / 2 .
\end{aligned}
$$

(5) For every $\mathfrak{p}>0$, we find $\mathfrak{c}, \mathfrak{e}_{0}>0$ such that, for all $\boldsymbol{p} \in \mathcal{B}_{\mathfrak{p}}, \mathfrak{e} \in\left(0, \mathfrak{e}_{0}\right]$, $\ell=1,2$, and $\boldsymbol{h} \in \mathbb{R}^{3}$, we have

$$
\left\|\left(U_{j} \partial_{\boldsymbol{h}}^{\ell} H_{j+1} U_{j}^{*}-\partial_{\boldsymbol{h}}^{\ell} H_{j}^{j+1}\right)\left(H_{\mathrm{f}}^{(j, j+1)}+\rho_{j}\right)^{-1 / 2}\right\| \leqslant \mathfrak{c e} \rho_{j}^{1 / 2}|\boldsymbol{h}|^{\ell} .
$$


Proof. (1)\&(2): Representing the absolute values by means of (2.58) and using a Neumann series expansion we deduce that

$$
\begin{aligned}
H_{m}^{j}(\boldsymbol{p}+\boldsymbol{h}) & =H_{m}^{j}(\boldsymbol{p})+{\underset{\tau-l i m}{\tau \rightarrow \infty}}_{-\tau}^{\tau}\left(R_{m}^{j}(\boldsymbol{p}+\boldsymbol{h}, i y)-R_{m}^{j}(\boldsymbol{p}, i y)\right) \frac{i y \mathrm{~d} y}{\pi} \\
& =\sum_{\ell=0}^{\infty} \frac{1}{\ell !} \partial_{\boldsymbol{h}}^{\ell} H_{m}^{j}(\boldsymbol{p}), \quad \text { on } \operatorname{dom}\left(H_{\mathrm{f}}^{(j)}\right),|\boldsymbol{h}|<1,
\end{aligned}
$$

where we define (again dropping all $\boldsymbol{p}$ 's so that $R_{m}^{j}(i y) \equiv R_{m}^{j}(\boldsymbol{p}, i y)$ )

$$
\begin{aligned}
\partial_{\boldsymbol{h}} H_{m}^{j} \psi & :=\lim _{\tau \rightarrow \infty} \int_{-\tau}^{\tau} R_{m}^{j}(i y) \boldsymbol{\alpha} \cdot \boldsymbol{h} R_{m}^{j}(i y) \psi \frac{y \mathrm{~d} y}{i \pi}, \quad \psi \in \operatorname{dom}\left(H_{\mathrm{f}}^{(j)}\right), \\
\partial_{\boldsymbol{h}}^{\ell} H_{m}^{j} & :=(-1)^{\ell+1} \ell ! \int_{\mathbb{R}} R_{m}^{j}(i y)\left\{\boldsymbol{\alpha} \cdot \boldsymbol{h} R_{m}^{j}(i y)\right\}^{\ell} \frac{y \mathrm{~d} y}{i \pi} \in \mathcal{B}\left(\mathscr{H}_{j}\right),
\end{aligned}
$$

where $\ell \geqslant 2$. In fact, since $\left\|\boldsymbol{\alpha} \cdot \boldsymbol{h} R_{m}^{j}(i y)\right\| \leqslant|\boldsymbol{h}|\left(1+y^{2}\right)^{-1 / 2}$ the integrals in (B.6) are absolutely convergent and one easily verifies $\left\|\partial_{\boldsymbol{h}}^{\ell} H_{m}^{j}\right\| \leqslant 2 \ell !|\boldsymbol{h}|^{\ell} / \pi(\ell-1)$, for $\ell \in \mathbb{N}, \ell \geqslant 2$. So, indeed, the part $\sum_{2}^{\infty} \ldots$ of the series in (B.4) converges in $\mathcal{B}\left(\mathscr{H}_{j}\right)$, if $|\boldsymbol{h}|<1$. It is then clear that the limit in (B.5) exists. Combining (A.11) with (B.4) we further infer that the closure of the symmetric operator $\partial_{\boldsymbol{h}} H_{m}^{j}$ - henceforth again denoted by the same symbol - is defined on a domain containing the domain of $\left|D_{m}^{j}\right|^{1 / 4}$ and $\left\|\partial_{\boldsymbol{h}} H_{m}^{j}\left|D_{m}^{j}\right|^{-1 / 4}\right\| \leqslant \mathfrak{c}|\boldsymbol{h}|+\mathcal{O}\left(|\boldsymbol{h}|^{2}\right)$; thus

$$
\left\|\partial_{\boldsymbol{h}} H_{m}^{j}\left(H_{m}^{j}\right)^{-1 / 4}\right\| \leqslant\left\|\partial_{\boldsymbol{h}} H_{m}^{j}\left|D_{m}^{j}\right|^{-1 / 4}\right\| \leqslant \mathfrak{c}|\boldsymbol{h}| .
$$

The first-order term in the series (B.4) is, therefore, an infinitesimal perturbation of the self-adjoint zeroth-order term $H_{m}^{j}$ and, as all higher-order terms are bounded, we conclude that the series (B.4) defines an extension of $\left\{H_{m}^{j}(\boldsymbol{p})\right\}$ to an analytic family of type $A$ defined on $\left\{\boldsymbol{z} \in \mathbb{C}^{3}:|\operatorname{Im} \boldsymbol{z}|<1\right\}$.

(3): We represent the difference of the $\ell$-th derivatives by means of (B.5) or (B.6) and rearrange the integrands in a telescopic sum of terms which are proportional to $\left(R_{j} \boldsymbol{\alpha} \cdot \boldsymbol{h}\right)^{n}\left(R_{j}-R_{m}^{j}\right)\left(\boldsymbol{\alpha} \cdot \boldsymbol{h} R_{m}^{j}\right)^{\ell-n}, n=0, \ldots, \ell$. Then we multiply the telescopic sum from the right by $\left(H_{\mathrm{f}}^{(m, j)}+\rho_{m}\right)^{-1 / 2}$, observe that $\left(\boldsymbol{\alpha} \cdot \boldsymbol{h} R_{m}^{j}\right)^{\ell-n}$ and $\left(H_{\mathrm{f}}^{(m, j)}+\rho_{m}\right)^{-1 / 2}$ commute, and estimate all terms by $\left\|R_{a}^{b}\right\| \leqslant 1 /\left(1+y^{2}\right)^{1 / 2}$ and (A.4). Each summand in the telescopic sum is absolutely integrable. (For $\ell=1$, we start with $\psi \in \operatorname{dom}\left(H_{\mathrm{f}}^{(j)}\right)$ and extend $\left(\partial_{\boldsymbol{h}} H_{j}-\partial_{\boldsymbol{h}} H_{m}^{j}\right)\left(H_{\mathrm{f}}^{(m, j)}+\rho_{m}\right)^{-1 / 2}$ to all of $\mathscr{H}_{j}$ by continuity, preserving the same symbol for the extension).

(4): By the additional assumption we know that $E_{m}^{j}$ and $\Pi_{m}^{j}$ depend analytically on $\boldsymbol{p}$ [21]. Then, by differentiating $\left\langle H_{m}^{j} \phi \mid \Pi_{m}^{j} \psi\right\rangle=E_{m}^{j}\left\langle\phi \mid \Pi_{m}^{j} \psi\right\rangle$ we obtain the following Leibniz formula, for all $\phi \in \operatorname{dom}\left(H_{\mathrm{f}}^{(j)}\right), \psi \in \mathscr{H}_{j}, \mu \in \mathbb{N}$, and $\boldsymbol{h} \in \mathbb{R}^{3}$,

$$
\sum_{\nu=0}^{\mu}\left(\begin{array}{l}
\mu \\
\nu
\end{array}\right)\left\langle\partial_{\boldsymbol{h}}^{\nu} H_{m}^{j} \phi \mid \partial_{\boldsymbol{h}}^{\mu-\nu} \Pi_{m}^{j} \psi\right\rangle=\sum_{\nu=0}^{\mu}\left(\begin{array}{l}
\mu \\
\nu
\end{array}\right)\left(\partial_{\boldsymbol{h}}^{\nu} E_{m}^{j}\right)\left\langle\phi \mid \partial_{\boldsymbol{h}}^{\mu-\nu} \Pi_{m}^{j} \psi\right\rangle .
$$


In view of $\operatorname{dom}\left(\partial_{\boldsymbol{h}}^{\nu} H_{m}^{j}\right) \supset \operatorname{dom}\left(H_{\mathrm{f}}^{(j)}\right)=\operatorname{dom}\left(H_{m}^{j}\right) \supset \operatorname{Ran}\left(\Pi_{m}^{j}\right)$ we infer from (B.8) by induction on $\mu$ that $\partial_{\boldsymbol{h}}^{\mu} \Pi_{m}^{j}: \mathscr{H}_{j} \rightarrow \operatorname{dom}\left(H_{m}^{j}\right)=\operatorname{dom}\left(H_{\mathrm{f}}^{(j)}\right)$ and, in particular,

$$
\begin{aligned}
\left(\partial_{\boldsymbol{h}} H_{m}^{j}-\partial_{\boldsymbol{h}} E_{m}^{j}\right) \Pi_{m}^{j}= & -\left(H_{m}^{j}-E_{m}^{j}\right) \partial_{\boldsymbol{h}} \Pi_{m}^{j} \\
\left(\partial_{\boldsymbol{h}}^{2} H_{m}^{j}\right) \Pi_{m}^{j}= & -2\left(\partial_{\boldsymbol{h}} H_{m}^{j}-\partial_{\boldsymbol{h}} E_{m}^{j}\right) \partial_{\boldsymbol{h}} \Pi_{m}^{j} \\
& +\left(\partial_{\boldsymbol{h}}^{2} E_{m}^{j}\right) \Pi_{m}^{j}-\left(H_{m}^{j}-E_{m}^{j}\right) \partial_{\boldsymbol{h}}^{2} \Pi_{m}^{j} .
\end{aligned}
$$

We deduce from the first line in (B.10) that

$$
\begin{aligned}
\left(\Pi_{m}^{j}\right)^{\perp} \partial_{\boldsymbol{h}} \Pi_{m}^{j} & =-\left(\mathcal{R}_{m}^{j}\right)^{\perp}\left(\partial_{\boldsymbol{h}} H_{m}^{j}\right) \Pi_{m}^{j}, \\
\left(\partial_{\boldsymbol{h}} E_{m}^{j}\right) \Pi_{m}^{j} & =\Pi_{m}^{j}\left(\partial_{\boldsymbol{h}} H_{m}^{j}\right) \Pi_{m}^{j} .
\end{aligned}
$$

Multiplying the second line in (B.10) by $\Pi_{m}^{j}$ and using (B.10) and $\operatorname{Tr}\left\{\Pi_{m}^{j}\right\}=4$ we arrive at (B.2).

(5): The proof is similar to that of (3). In fact, by definition we have the relation $\check{R}_{j+1}(i y)=U_{j} R_{j+1}(i y) U_{j}^{*}$ which implies the following analogs of (B.5) and (B.6):

$$
\begin{aligned}
U_{j} \partial_{\boldsymbol{h}} H_{j+1} U_{j}^{*} \psi & =\lim _{\tau \rightarrow \infty} \int_{-\tau}^{\tau} \check{R}_{j+1}(i y) \boldsymbol{\alpha} \cdot \boldsymbol{h} \check{R}_{j+1}(i y) \psi \frac{y \mathrm{~d} y}{i \pi}, \\
U_{j} \partial_{\boldsymbol{h}}^{2} H_{j+1} U_{j}^{*} & =2 \int_{\mathbb{R}} \check{R}_{j+1}(i y)\left\{\boldsymbol{\alpha} \cdot \boldsymbol{h} \check{R}_{j+1}(i y)\right\}^{2} \frac{i y \mathrm{~d} y}{\pi},
\end{aligned}
$$

where $\psi \in \operatorname{dom}\left(H_{\mathrm{f}}^{(j+1)}\right)$. As before we employ (B.5) and (B.6) themselves to represent derivatives of $H_{j}^{j+1}$. Let

$$
\Delta_{j}(y):=\check{R}_{j+1}(i y)-R_{j}^{j+1}(i y) .
$$

Then a brief computation using (2.37) and Lemma A.2 yields

$$
\left(\check{D}_{j+1}-i y\right) \Delta_{j}(y)=\boldsymbol{\alpha} \cdot\left\{\mathfrak{e}^{2} \check{\boldsymbol{c}}_{j}-\mathfrak{e} \varphi\left(\boldsymbol{F}_{j}^{j+1}\right)\right\} R_{j}^{j+1}(i y)
$$

on $\operatorname{dom}\left(H_{\mathrm{f}}^{(j+1)}\right)$. Applying (2.34) and Lemma A.2 we conclude that

$$
\left\|\Delta_{j}(y)\left(H_{\mathrm{f}}^{(j, j+1)}+\rho_{j}\right)^{-1 / 2}\right\| \leqslant \mathfrak{c} \mathfrak{e} \rho_{j}^{1 / 2}\left(1+y^{2}\right)^{-1} .
$$

Now, we follow literally the steps described in the proof of Part (3) using (B.13) as an analog (in fact special case) of (A.4) to arrive at (B.3).

\section{Appendix C. Comparison of $\boldsymbol{H}_{j}^{j+1}$ and $\check{H}_{j+1}$}

\section{C.1. Proof of Lemma 4.1}

The proof Lemma 4.1 uses heavily cancellations which follow from (2.35). 
Proof. Let $\vartheta \in \operatorname{dom}\left(H_{\mathrm{f}}^{(j)}\right)$. Using the abbreviation (B.11) we have

$$
I:=\left\{\left|\check{D}_{j+1}\right|-\left|D_{j}^{j+1}\right|\right\} \vartheta \otimes \Omega_{j}^{j+1}=\lim _{\tau \rightarrow \infty} \int_{-\tau}^{\tau} \Delta_{j}(y)\left(\vartheta \otimes \Omega_{j}^{j+1}\right) \frac{i y \mathrm{~d} y}{\pi} .
$$

In view of (B.12) we further have

$$
\Delta_{j}(y)\left(\vartheta \otimes \Omega_{j}^{j+1}\right)=\check{R}_{j+1}(i y) \boldsymbol{\alpha}\left(R_{j}(i y) \vartheta\right) \otimes\left\{\mathfrak{e}^{2} \check{\boldsymbol{c}}_{j} \Omega_{j}^{j+1}-\mathfrak{e}\left|\boldsymbol{F}_{j}^{j+1}\right\rangle\right\} .
$$

We recall that $\left|\check{\boldsymbol{c}}_{j}\right|,\left\|\boldsymbol{F}_{j}^{j+1}\right\| \leqslant \mathfrak{c} \rho_{j},\left\|R_{a}^{b}(i y)\right\| \leqslant\left(1+y^{2}\right)^{-1 / 2}$, and observe that the resolvent $R_{j}^{j+1}(i y)$ leaves the subspaces $\mathscr{X}_{n} \equiv \mathscr{X}_{n}^{(j, j+1)}, n=0,1, \ldots$, defined in (2.26), invariant. Moreover, the restriction of the field operator $\varphi\left(\boldsymbol{F}_{j}^{j+1}\right)$ is bounded as a map from $\mathscr{X}_{0} \oplus \mathscr{X}_{1}$ to $\mathscr{X}_{0} \oplus \mathscr{X}_{1} \oplus \mathscr{X}_{2}$, with norm $\leqslant \mathfrak{c}\left\|\boldsymbol{F}_{j}^{j+1}\right\| \leqslant$ $\mathfrak{c}^{\prime} \rho_{j}$. Taking all these remarks into account we infer from (B.12) that

$$
\| \Delta_{j}(y)\left\lceil\mathscr{X}_{0} \oplus \mathscr{X}_{1} \| \leqslant \mathfrak{c} \mathfrak{e} \rho_{j}\left(1+y^{2}\right)^{-1} .\right.
$$

Rearranging (C.2) using $R_{j}^{j+1}\left(\psi \otimes \Omega_{j}^{j+1}\right)=\left(R_{j} \psi\right) \otimes \Omega_{j}^{j+1}$ we further obtain

$$
\begin{aligned}
\Delta_{j}(y)\left(\vartheta \otimes \Omega_{j}^{j+1}\right) & =\left(R_{j}(i y) \boldsymbol{\alpha} R_{j}(i y) \vartheta\right) \otimes\left\{\mathfrak{e}^{2} \check{\boldsymbol{c}}_{j} \Omega_{j}^{j+1}\right\}-V+W, \\
V & :=R_{j}^{j+1}(i y) \boldsymbol{\alpha}\left(R_{j}(i y) \vartheta\right) \otimes\left|\mathfrak{e} \boldsymbol{F}_{j}^{j+1}\right\rangle, \\
W & :=\Delta_{j}(y) \boldsymbol{\alpha}\left(R_{j}(i y) \vartheta\right) \otimes\left\{\mathfrak{e}^{2} \check{\boldsymbol{c}}_{j} \Omega_{j}^{j+1}-\mathfrak{e}\left|\boldsymbol{F}_{j}^{j+1}\right\rangle\right\} .
\end{aligned}
$$

We represent $V \in \mathscr{X}_{1}$ as $V=\{V(k)\} \in L^{2}\left[\mathcal{A}_{j}^{j+1}, \mathscr{H}_{j}\right]$ with

$$
V(k)=\mathfrak{e} \boldsymbol{F}_{j}^{j+1}(k) R_{j}(\boldsymbol{p}-\boldsymbol{k}, i y) \boldsymbol{\alpha} R_{j}(\boldsymbol{p}, i y) \vartheta, \quad \text { a.e. } k \in \mathcal{A}_{j}^{j+1} .
$$

Since $|\boldsymbol{k}| \leqslant \rho_{j}$, for $k \in \mathcal{A}_{j}^{j+1}$, the resolvent identity implies $R_{j}(\boldsymbol{p}-\boldsymbol{k}, i y)=$ $R_{j}(\boldsymbol{p}, i y)+\mathcal{O}\left(\rho_{j}\left(1+y^{2}\right)^{-1}\right)$, and together with $\left\|\boldsymbol{F}_{j}^{j+1}\right\| \leqslant \mathfrak{c} \rho_{j}$ this gives

$$
V=\left(R_{j}(i y) \boldsymbol{\alpha} R_{j}(i y) \vartheta\right) \otimes\left|\mathfrak{e} \boldsymbol{F}_{j}^{j+1}\right\rangle+\mathcal{O}\left(\mathfrak{e} \rho_{j}^{2}\left(1+y^{2}\right)^{-3 / 2}\|\vartheta\|\right) .
$$

The inequality (C.3) applied to the third member on the RHS of (C.4) yields

$$
\begin{aligned}
\|W\| & \leqslant \mathfrak{c} \mathfrak{e} \rho_{j}\left(1+y^{2}\right)^{-1}\left\|R_{j}(i y) \vartheta\right\|\left\{\mathfrak{e}^{2}\left|\check{\boldsymbol{c}}_{j}\right|+\mathfrak{e}\left\|\boldsymbol{F}_{j}^{j+1}\right\|\right\} \\
& \leqslant \mathfrak{c}^{\prime} \mathfrak{e}^{2} \rho_{j}^{2}\left(1+y^{2}\right)^{-3 / 2}\|\vartheta\| .
\end{aligned}
$$

Altogether, employing the formula (B.5) for the derivative of $H_{j}$ and using $\int_{\mathbb{R}} d y /\left(1+y^{2}\right)<\infty$, we see that the term in (C.1) can be written as

$$
I=\left(\nabla H_{j} \vartheta\right) \otimes\left\{\mathfrak{e}\left|\boldsymbol{F}_{j}^{j+1}\right\rangle-\mathfrak{e}^{2} \check{\boldsymbol{c}}_{j} \Omega_{j}^{j+1}\right\}+\mathcal{O}\left(\mathfrak{e} \rho_{j}^{2}\|\vartheta\|\right) .
$$

But we have $\left(\check{H}_{j+1}-H_{j}^{j+1}\right)\left(\vartheta \otimes \Omega_{j}^{j+1}\right)=I+I I$ with

$$
I I:=\left(\check{H}_{\mathrm{f}}^{(j+1)}-H_{\mathrm{f}}^{(j+1)}\right)\left(\vartheta \otimes \Omega_{j}^{j+1}\right)=\mathfrak{e} \vartheta \otimes\left\{\mathfrak{e}\left\|\omega^{1 / 2} f_{j}^{j+1}\right\|^{2} \Omega_{j}^{j+1}-\left|\omega f_{j}^{j+1}\right\rangle\right\} .
$$

Applying these formulas for every $\vartheta \in \operatorname{Ran}\left(\Pi_{j}\right) \subset \operatorname{dom}\left(H_{\mathrm{f}}^{(j)}\right)$ we arrive at

$$
\begin{aligned}
\left(\check{H}_{j+1}-H_{j}^{j+1}\right) \Pi_{j}^{j+1}= & \mathfrak{e} \nabla H_{j} \Pi_{j} \otimes\left|\boldsymbol{F}_{j}^{j+1}\right\rangle\left\langle\Omega_{j}^{j+1}\left|-\mathfrak{e} \Pi_{j} \otimes\right| \omega f_{j}^{j+1}\right\rangle\left\langle\Omega_{j}^{j+1}\right| \\
& +\left(\mathfrak{e}^{2}\left\langle f_{j}^{j+1} \mid \omega f_{j}^{j+1}\right\rangle-\mathfrak{e}^{2} \check{\boldsymbol{c}}_{j} \cdot \nabla H_{j}\right) \Pi_{j}^{j+1}+\mathcal{O}\left(\mathfrak{e} \rho_{j}^{2}\right),
\end{aligned}
$$

and we conclude by means of (2.35) and $\nabla H_{j} \Pi_{j}=\nabla E_{j} \Pi_{j}+\Pi_{j}^{\perp} \nabla H_{j} \Pi_{j}$. 


\section{C.2. Proof of Lemma 4.2}

In this subsection we give the proof of a Lemma in which the resolvents of $\check{H}_{j+1}$ and $H_{j}^{j+1}$ are compared. We use the notation

$$
\mathscr{R}_{j}(\boldsymbol{p}, \boldsymbol{k}):=\left(H_{j}(\boldsymbol{p}-\boldsymbol{k})-E_{j}(\boldsymbol{p})+|\boldsymbol{k}|\right)^{-1} .
$$

in the proof. This operator occurs in the direct integral representation of $\mathcal{R}_{j}^{j+1}(\boldsymbol{p})$ restricted to $\mathscr{X}_{1}^{(j, j+1)}$,

$$
\left.\mathcal{R}_{j}^{j+1}(\boldsymbol{p})\right|_{\mathscr{X}_{1}^{(j, j+1)}}=\int_{\mathcal{A}_{j}^{j+1}}^{\oplus} \mathscr{R}_{j}(\boldsymbol{p}, \boldsymbol{k}) \mathrm{d} k .
$$

We recall that the resolvents $\mathcal{R}_{j}^{j+1}, \check{\mathcal{R}}_{j+1}$, and $\check{\mathcal{R}}_{j+1}^{\perp}$ are defined in $(2.24)$, (2.39), and (2.40), respectively. The resolvents $\mathcal{R}_{j}$ and $\mathcal{R}_{j}^{\perp}$ are introduced in and below Eq. (2.25).

Proof. (1): On account of (3.17) and $|\boldsymbol{k}| \geqslant \rho_{j+1}=\rho_{j} / 2$ we have

$$
r_{j}(\boldsymbol{p}, \boldsymbol{k}):=E_{j}(\boldsymbol{p}-\boldsymbol{k})-E_{j}(\boldsymbol{p})+|\boldsymbol{k}| \geqslant \rho_{j} / 4 .
$$

Hence, $E_{j}(\boldsymbol{p})-|\boldsymbol{k}|$ belongs to the resolvent set of both $H_{j}(\boldsymbol{p}-\boldsymbol{k})$ and $H_{j}(\boldsymbol{p})$ and (A.11) together with the second resolvent identity implies

$$
\left\|\left(\mathscr{R}_{j}(\boldsymbol{p}, \boldsymbol{k})-\mathcal{R}_{j}(\boldsymbol{p},|\boldsymbol{k}|)\right) \Psi\right\| \leqslant \mathfrak{c}|\boldsymbol{k}|\left\|H_{j}(\boldsymbol{p}-\boldsymbol{k})^{1 / 4} \mathscr{R}_{j}(\boldsymbol{p}, \boldsymbol{k})\right\|\left\|\mathcal{R}_{j}(\boldsymbol{p},|\boldsymbol{k}|) \Psi\right\|,
$$

for all $\Psi \in \mathscr{H}_{j}$. Here $|\boldsymbol{k}| \leqslant \rho_{j}$ and, by the spectral calculus, the first norm on the RHS is not greater than $E_{j}(\boldsymbol{p}-\boldsymbol{k})^{1 / 4} / r_{j}(\boldsymbol{p}, \boldsymbol{k}) \leqslant \mathfrak{c}(\mathfrak{p}) / \rho_{j}$. Choosing $\Psi:=\Pi_{j}^{\perp} \psi$ we obtain (4.1) since, of course, $\left\|\mathcal{R}_{j}(\boldsymbol{p},|\boldsymbol{k}|) \Pi_{j}^{\perp} \psi\right\| \leqslant\left\|\mathcal{R}_{j}^{\perp}(\boldsymbol{p}) \psi\right\|$.

(2): We may apply Lemma A.3 with $K_{j}^{j+1}=H_{j}^{j+1}$ and $K_{j+1}=\check{H}_{j+1}$. Indeed, let $t \geqslant 0, \varepsilon>0$, and $z:=t+i \varepsilon$. Then $\delta_{j}=\mathcal{O}\left(\mathfrak{e} \rho_{j}\right),\left|E_{j}-E_{j+1}\right|=$ $\mathcal{O}\left(\mathfrak{e} \rho_{j}\right)$, the first inequality in (A.5), and $\left|D_{j}^{j+1}\right| \geqslant 1$ imply

$$
\begin{aligned}
N_{z}^{(1)}:= & \left\|\check{\Pi}_{j+1}^{\perp}\left(\check{\mathcal{R}}_{j+1}\left(z+\delta_{j}\right)-\mathcal{R}_{j}^{j+1}(z)\right)\left(\Pi_{j}^{j+1}\right)^{\perp} \psi\right\| \\
\leqslant & \mathfrak{c} \mathfrak{e} \rho_{j}^{1 / 2}\left\|\check{\Pi}_{j+1}^{\perp} \check{\mathcal{R}}_{j+1}\left(z+\delta_{j}\right)\right\| \\
& \cdot\left\|\left|D_{j}^{j+1}\right|^{1 / 2}\left(H_{\mathrm{f}}^{(j, j+1)}+\rho_{j}\right)^{1 / 2}\left(\Pi_{j}^{j+1}\right)^{\perp} \mathcal{R}_{j}^{j+1}(z) \psi\right\| .
\end{aligned}
$$

Here, (3.17) and (3.21) permit to get

$$
\left\|\check{\mathcal{R}}_{j+1}\left(z+\delta_{j}\right) \check{\Pi}_{j+1}^{\perp}\right\| \leqslant\left(\operatorname{gap}_{j+1}+\delta_{j}+t\right)^{-1} \leqslant \mathfrak{c}\left(\rho_{j}+t\right)^{-1},
$$

uniformly in $\varepsilon>0$ and small $\mathfrak{e}>0$. Moreover, $\left(H_{\mathrm{f}}^{(j, j+1)}+\rho_{j}\right)^{1 / 2}$ commutes strongly with $\left(\Pi_{j}^{j+1}\right)^{\perp}$ and $\mathcal{R}_{j}^{j+1}(z)$. Since also $\left|D_{j}^{j+1}\right| \leqslant\left(H_{j}^{j+1}-E_{j}\right)+E_{j}$ and $E_{j} \leqslant \mathfrak{c}(\mathfrak{p})$ on $\overline{\mathcal{B}}_{\mathfrak{p}}$ we deduce that

$$
\begin{aligned}
\theta & :=\lim _{\varepsilon \searrow 0}\left\|\left|D_{j}^{j+1}\right|^{1 / 2}\left(H_{\mathrm{f}}^{(j, j+1)}+\rho_{j}\right)^{1 / 2}\left(\Pi_{j}^{j+1}\right)^{\perp} \mathcal{R}_{j}^{j+1}(z) \psi\right\| \\
& \leqslant\left\|\left(H_{\mathrm{f}}^{(j, j+1)}+\rho_{j}\right)^{1 / 2}\left(\left(\mathcal{R}_{j}^{j+1}\right)^{\perp}\right)^{1 / 2} \psi\right\|+\mathfrak{c}\left\|\left(H_{\mathrm{f}}^{(j, j+1)}+\rho_{j}\right)^{1 / 2}\left(\mathcal{R}_{j}^{j+1}\right)^{\perp} \psi\right\| .
\end{aligned}
$$


Next, we use that $\psi$ and, hence, $\left(\left(\mathcal{R}_{j}^{j+1}\right)^{\perp}\right)^{s} \psi$, belong to $\mathscr{X}_{0}^{(j, j+1)} \oplus \mathscr{X}_{1}^{(j, j+1)}$ and that $\eta \in \mathscr{X}_{0}^{(j, j+1)} \oplus \mathscr{X}_{1}^{(j, j+1)}$ entails $\left\|\left(H_{\mathrm{f}}^{(j, j+1)}+\rho_{j}\right)^{1 / 2} \eta\right\| \leqslant\left(2 \rho_{j}\right)^{1 / 2}\|\eta\|$. Taking this observation into account we obtain

$$
\theta \leqslant \mathfrak{c} \rho_{j}^{1 / 2}\left(\left\|\left(\left(\mathcal{R}_{j}^{j+1}\right)^{\perp}\right)^{1 / 2} \psi\right\|+\left\|\left(\mathcal{R}_{j}^{j+1}\right)^{\perp} \psi\right\|\right) \leqslant \mathfrak{c}^{\prime} \rho_{j}^{1 / 2}\left(\|\psi\|+\left\|\left(\mathcal{R}_{j}^{j+1}\right)^{\perp} \psi\right\|\right) .
$$

Altogether we arrive at

$$
\lim _{\varepsilon \searrow 0} N_{t+i \varepsilon}^{(1)} \leqslant \mathfrak{c} \mathfrak{e} \rho_{j}\left(\rho_{j}+t\right)^{-1}\left(\|\psi\|+\left\|\left(\mathcal{R}_{j}^{j+1}\right)^{\perp} \psi\right\|\right),
$$

for every $\psi \in \mathscr{X}_{0}^{(j, j+1)} \oplus \mathscr{X}_{1}^{(j, j+1)}$. For $t=0$, this is (4.2) with $s=1$, which immediately implies (4.3).

Let $N_{0}^{(1 / 2)}$ denote the LHS of (4.2) with $s=1 / 2$. We have $\check{\mathcal{R}}_{j+1}^{\perp}\left(\delta_{j}\right)^{1 / 2}=$ $\int_{0}^{\infty} \check{\mathcal{R}}_{j+1}^{\perp}\left(t+\delta_{j}\right) t^{-1 / 2} d t / \pi$ and an analogous representation of the square root of $\left(\mathcal{R}_{j}^{j+1}\right)^{\perp}$. Therefore,

$$
N_{0}^{(1 / 2)} \leqslant \int_{0}^{\infty} N_{t}^{(1)} \frac{\mathrm{d} t}{\pi t^{1 / 2}} \leqslant \mathfrak{c} \mathfrak{e} \int_{0}^{\infty} \frac{\rho_{j}}{\rho_{j}+t} \frac{\mathrm{d} t}{\pi t^{1 / 2}}\left(\|\psi\|+\left\|\left(\mathcal{R}_{j}^{j+1}\right)^{\perp} \psi\right\|\right),
$$

which is (4.2) with $s=1 / 2$, as the integral on the RHS equals $\rho_{j}^{1 / 2}$.

\section{Appendix D. A Formula for $a(k) \phi_{j}$}

In this section we derive the formula for $a(k) \phi_{j}$ stated in Lemma 6.1 where $\phi_{j}$ is a ground state eigenvector of $H_{j}$. We will frequently use the abbreviation

$$
\mathscr{R}_{j}(\boldsymbol{p}, \boldsymbol{k}):=\left(H_{j}(\boldsymbol{p}-\boldsymbol{k})-E_{j}(\boldsymbol{p})+|\boldsymbol{k}|\right)^{-1} .
$$

Proof of Lemma 6.1. Let $f \in C_{0}^{\infty}\left[\left\{|\boldsymbol{k}| \geqslant \rho_{j}\right\}\right], \boldsymbol{q} \in \mathbb{R}^{3} \backslash\{\mathbf{0}\}$, and $\mu \in \mathbb{Z}_{2}$. Moreover, let

$$
\tilde{f}(k):=f(\boldsymbol{k}) \delta_{\lambda, \mu}, \quad k=(\boldsymbol{k}, \lambda) \in \mathbb{R}^{3} \times \mathbb{Z}_{2} .
$$

We fix $\boldsymbol{p} \in \mathbb{R}^{3}$ and the scale parameter $j \in \mathbb{N} \cup\{\infty\}$, and abbreviate $D:=$ $D_{j}(\boldsymbol{p}), D_{\boldsymbol{q}}:=D_{j}(\boldsymbol{p}-\boldsymbol{q}), R(i y):=(D-i y)^{-1}, R_{\boldsymbol{q}}(i y):=\left(D_{\boldsymbol{q}}-i y\right)^{-1}$.

Finally, we recall that $\boldsymbol{m}$ denotes multiplication with $\boldsymbol{k}$.

On the dense domain $\operatorname{dom}\left(\left(H_{\mathrm{f}}^{(j)}\right)^{3 / 2}\right)$ we then have the operator identities

$$
\left[H_{\mathrm{f}}^{(j)}, a^{\dagger}(\tilde{f}),\right]=a^{\dagger}(\omega \tilde{f}), \quad\left[\mathbf{p}_{\mathrm{f}}^{(j)}, a^{\dagger}(\tilde{f})\right]=a^{\dagger}(\boldsymbol{m} \tilde{f}),
$$

and, hence,

$$
D a^{\dagger}(\tilde{f})-a^{\dagger}(\tilde{f}) D_{\boldsymbol{q}}=\boldsymbol{\alpha} \cdot\left\{\mathfrak{e}\langle\boldsymbol{G} \mid \tilde{f}\rangle-a^{\dagger}((\boldsymbol{m}-\boldsymbol{q}) \tilde{f})\right\} .
$$

Thanks to Lemma A.2 we know that $R_{\boldsymbol{q}}(i y)$ maps $\operatorname{dom}\left(\left(H_{\mathrm{f}}^{(j)}\right)^{3 / 2}\right)$ into itself. On that domain we thus have

$$
a^{\dagger}(\tilde{f}) R_{\boldsymbol{q}}(i y)-R(i y) a^{\dagger}(\tilde{f})=R(i y) \boldsymbol{\alpha} \cdot\left\{\mathfrak{e}\langle\boldsymbol{G} \mid \tilde{f}\rangle-a^{\dagger}((\boldsymbol{m}-\boldsymbol{q}) \tilde{f})\right\} R_{\boldsymbol{q}}(i y) .
$$


Since $\phi_{j} \in \operatorname{dom}\left(H_{\mathrm{f}}^{(j)}\right)$, the previous relation implies, for all $\eta^{\prime} \in \mathscr{C}_{j}$,

$$
\begin{aligned}
\Delta\left(\boldsymbol{q}, \tilde{f}, \eta^{\prime}\right):= & \left\langle\left|D_{\boldsymbol{q}}\right| \eta^{\prime} \mid a(\tilde{f}) \phi_{j}\right\rangle-\left\langle a^{\dagger}(\tilde{f}) \eta^{\prime}|| D \mid \phi_{j}\right\rangle \\
= & \lim _{\tau \rightarrow \infty} \int_{-\tau}^{\tau}\left\langle R(i y) \boldsymbol{\alpha} \cdot a^{\dagger}((\boldsymbol{m}-\boldsymbol{q}) \tilde{f}) R_{\boldsymbol{q}}(i y) \eta^{\prime} \mid \phi_{j}\right\rangle \frac{i y \mathrm{~d} y}{\pi} \\
& -\mathfrak{e}\langle\boldsymbol{G} \mid \tilde{f}\rangle \cdot \lim _{\tau \rightarrow \infty} \int_{-\tau}^{\tau}\left\langle R(i y) \boldsymbol{\alpha} R_{\boldsymbol{q}}(i y) \eta^{\prime} \mid \phi_{j}\right\rangle \frac{i y \mathrm{~d} y}{\pi}
\end{aligned}
$$

where we used the representation (2.58) of the absolute value. In the integral appearing in the second line we now write $R_{\boldsymbol{q}}(i y) y / i=\mathbb{1}-D_{\boldsymbol{q}} R_{\boldsymbol{q}}(i y)$ and apply the formula $(2.57)$ for the sign function $S:=\operatorname{sgn}(D)$. In the third line we expand the right resolvent as $R_{\boldsymbol{q}}=R+R \boldsymbol{\alpha} \cdot \boldsymbol{q} R_{\boldsymbol{q}}$ and apply the formula (B.5) for the derivative of the Hamiltonian. Proceeding in the way we arrive at

$$
\begin{aligned}
\Delta\left(\boldsymbol{q}, \tilde{f}, \eta^{\prime}\right)= & \left\langle S \boldsymbol{\alpha} \cdot a^{\dagger}((\boldsymbol{m}-\boldsymbol{q}) \tilde{f}) \eta^{\prime} \mid \phi_{j}\right\rangle-J\left(\boldsymbol{q}, \tilde{f}, \eta^{\prime}\right) \\
& -\mathfrak{e}\langle\tilde{f} \mid \boldsymbol{G}\rangle \cdot\left\{\left\langle\eta^{\prime} \mid \nabla H_{j} \phi_{j}\right\rangle+\boldsymbol{I}\left(\boldsymbol{q}, \eta^{\prime}\right)\right\} .
\end{aligned}
$$

Here and below $\nabla H_{j}$ is evaluated at $\boldsymbol{p}$ and

$$
\begin{aligned}
\boldsymbol{I}\left(\boldsymbol{q}, \eta^{\prime}\right) & :=\int_{\mathbb{R}}\left\langle R(i y) \boldsymbol{\alpha} R(i y)(\boldsymbol{\alpha} \cdot \boldsymbol{p}) R_{\boldsymbol{q}}(i y) \eta^{\prime} \mid \phi_{j}\right\rangle \frac{i y \mathrm{~d} y}{\pi}, \\
J\left(\boldsymbol{q}, \tilde{f}, \eta^{\prime}\right) & :=\int_{\mathbb{R}}\left\langle R_{\boldsymbol{q}}(i y) D_{\boldsymbol{q}} \eta^{\prime} \mid \boldsymbol{\alpha} \cdot a((\boldsymbol{m}-\boldsymbol{q}) \tilde{f}) R(-i y) \phi_{j}\right\rangle \frac{\mathrm{d} y}{\pi} .
\end{aligned}
$$

By means of (A.2) it is easy to see that the latter integral is absolutely convergent. In fact, let $\rho>1$ be sufficiently large and set $\Theta:=H_{\mathrm{f}}^{(j)}+\rho$ and $B_{\boldsymbol{q}}:=\boldsymbol{\alpha} \cdot a((\boldsymbol{m}-\boldsymbol{q}) \tilde{f}) \Theta^{-1 / 2}$. Then (A.2) shows that the composition $F(y):=\Theta^{1 / 2} R(-i y) \Theta^{-1 / 2}$ is well defined on $\mathscr{H}_{j}$ and $\|F(y)\| \leqslant \mathfrak{c}\left(1+y^{2}\right)^{-1 / 2}$. Moreover, $\left\|B_{\boldsymbol{q}}\right\| \leqslant \mathfrak{c}\left\|(1+1 / \omega)^{1 / 2}(\boldsymbol{m}-\boldsymbol{q}) \tilde{f}\right\|$ by a standard estimate. From the representation

$$
J\left(\boldsymbol{q}, \tilde{f}, \eta^{\prime}\right)=\int_{\mathbb{R}}\left\langle\left\{D_{\boldsymbol{q}} R_{\boldsymbol{q}}(i y)\left|D_{\boldsymbol{q}}\right|^{-1 / 2}\right\}\left|D_{\boldsymbol{q}}\right|^{1 / 2} \eta^{\prime} \mid B_{\boldsymbol{q}} F(y) \Theta^{1 / 2} \phi_{j}\right\rangle \frac{\mathrm{d} y}{\pi},
$$

where the operator in curly brackets satisfies $\|\{\cdots\}\| \leqslant \mathfrak{c}\left(1+y^{2}\right)^{-1 / 4}$; we may thus read off that the map $\mathscr{C}_{j} \ni \eta^{\prime} \mapsto J\left(\boldsymbol{q}, \tilde{f}, \eta^{\prime}\right)$ is continuous when $\mathscr{C}_{j}$ is equipped with the form norm of $H_{\boldsymbol{q}}:=H_{j}(\boldsymbol{p}-\boldsymbol{q})$. Since $\mathscr{C}_{j}$ is a form core for $H_{\boldsymbol{q}}$ we may hence extend the definition of $J\left(\boldsymbol{q}, \tilde{f}, \eta^{\prime}\right)$ to all $\eta^{\prime} \in \mathcal{Q}\left(H_{\boldsymbol{q}}\right)$.

We further find by means of (D.1) and a virial type argument

$$
\begin{aligned}
& \left\langle\left(H_{\boldsymbol{q}}-E_{j}(\boldsymbol{p})+|\boldsymbol{q}|\right) \eta^{\prime} \mid a(\tilde{f}) \phi_{j}\right\rangle \\
& \quad=\left\langle H_{\boldsymbol{q}} \eta^{\prime} \mid a(\tilde{f}) \phi_{j}\right\rangle-\left\langle a^{\dagger}(\tilde{f}) \eta^{\prime} \mid H_{j}(\boldsymbol{p}) \phi_{j}\right\rangle+|\boldsymbol{q}|\left\langle\eta^{\prime} \mid a(\tilde{f}) \phi_{j}\right\rangle \\
& \quad=\Delta\left(\boldsymbol{q}, \tilde{f}, \eta^{\prime}\right)+\left\langle\eta^{\prime} \mid a((|\boldsymbol{q}|-\omega) \tilde{f}) \phi_{j}\right\rangle .
\end{aligned}
$$


Since $\phi_{j} \in \operatorname{dom}\left(H_{\mathrm{f}}^{(j)}\right)$ we know that $a(\tilde{f}) \phi_{j} \in \mathcal{Q}\left(H_{\mathrm{f}}^{(j)}\right)$ and, according to Lemma A.3(iv), the form domain of $H_{\boldsymbol{q}}$ is $\mathcal{Q}\left(H_{\mathrm{f}}^{(j)}\right)$. Taking this and the above remarks on $J\left(\boldsymbol{q}, \tilde{f}, \eta^{\prime}\right)$ into account we conclude that the first and the last line of (D.2) are continuous in $\eta^{\prime}$ w.r.t. the form norm of $H_{\boldsymbol{q}}$. Approximating $\mathscr{R}_{\boldsymbol{q}} \eta:=\mathscr{R}_{j}(\boldsymbol{p}, \boldsymbol{q}) \eta$, where $\eta \in \mathscr{H}_{j}$ is arbitrary, by some sequence in $\mathscr{C}_{j}$, which is convergent w.r.t. the form norm of $H_{p}$; we thus obtain

$$
\left\langle\eta \mid a(\tilde{f}) \phi_{j}\right\rangle=\Delta\left(\boldsymbol{q}, \tilde{f}, \mathscr{R}_{\boldsymbol{q}} \eta\right)+\left\langle\eta \mid \mathscr{R}_{\boldsymbol{q}} a((|\boldsymbol{q}|-\omega) \tilde{f}) \phi_{j}\right\rangle .
$$

Now, let $\tilde{f}_{\boldsymbol{q}, \varepsilon}(k):=h_{\varepsilon}(\boldsymbol{k}-\boldsymbol{q}) \delta_{\lambda, \mu}, h_{\varepsilon}(\boldsymbol{k}):=h(\boldsymbol{k} / \varepsilon) / \varepsilon^{3}$, for tiny $\varepsilon>0$, where $h \in C_{0}^{\infty}[\{|\boldsymbol{k}| \leqslant 1\},[0, \infty)]$ satisfies $\int_{\mathbb{R}^{3}} h=1$. In the next step we insert the peak function $f_{\boldsymbol{q}, \varepsilon}$ into (D.3), multiply the resulting expressions with $g \in$ $C_{0}^{\infty}\left[\mathbb{R}^{3} \backslash\{0\}\right]$, and integrate with respect to $\boldsymbol{q}$. Proceeding in this way we arrive at

$$
\int_{\mathbb{R}^{3}} g(\boldsymbol{q})\left\langle\eta \mid a\left(\tilde{f}_{\boldsymbol{q}, \varepsilon}\right) \phi_{j}\right\rangle \mathrm{d}^{3} \boldsymbol{q}=\sum_{\ell=1}^{5} C_{\ell}(\varepsilon),
$$

with (in $C_{3}(\varepsilon)$ we have $S \phi_{j} \in \operatorname{dom}\left(a\left((\boldsymbol{m}-\boldsymbol{q}) \tilde{f}_{\boldsymbol{q}, \varepsilon}\right)\right)$ because of (A.3))

$$
\begin{aligned}
& C_{1}(\varepsilon):=-\mathfrak{e} \int_{\mathbb{R}^{3}} g(\boldsymbol{q})\left\langle\tilde{f}_{\boldsymbol{q}, \varepsilon} \mid \boldsymbol{G}\right\rangle\left\langle\eta \mid \mathscr{R}_{\boldsymbol{q}} \nabla H_{j} \phi_{j}\right\rangle \mathrm{d}^{3} \boldsymbol{q}, \\
& C_{2}(\varepsilon):=\int_{\mathbb{R}^{3}} g(\boldsymbol{q})\left\langle\eta \mid \mathscr{R}_{\boldsymbol{q}} a\left((|\boldsymbol{q}|-\omega) \tilde{f}_{\boldsymbol{q}, \varepsilon}\right) \phi_{j}\right\rangle \mathrm{d}^{3} \boldsymbol{q}, \\
& C_{3}(\varepsilon):=\int_{\mathbb{R}^{3}} g(\boldsymbol{q})\left\langle\eta \mid \mathscr{R}_{\boldsymbol{q}} \boldsymbol{\alpha} \cdot a\left((\boldsymbol{m}-\boldsymbol{q}) \tilde{f}_{\boldsymbol{q}, \varepsilon}\right) S \phi_{j}\right\rangle \mathrm{d}^{3} \boldsymbol{q}, \\
& C_{4}(\varepsilon):=-\mathfrak{e} \int_{\mathbb{R}^{3}} g(\boldsymbol{q})\left\langle\tilde{f}_{\boldsymbol{q}, \varepsilon} \mid \boldsymbol{G}\right\rangle \cdot \boldsymbol{I}\left(\boldsymbol{q}, \mathscr{R}_{\boldsymbol{q}} \eta\right) \mathrm{d}^{3} \boldsymbol{q}, \\
& C_{5}(\varepsilon):=\int_{\mathbb{R}^{3}} g(\boldsymbol{q}) J\left(\boldsymbol{q}, \tilde{f}_{\boldsymbol{q}, \varepsilon}, \mathscr{R}_{\boldsymbol{q}} \eta\right) \mathrm{d}^{3} \boldsymbol{q} .
\end{aligned}
$$

It is straightforward to see that the LHS of (D.4) converges to $\left\langle\eta \mid a(\bar{g}) \phi_{j}\right\rangle$, as $\varepsilon>0$ tends to zero. Furthermore, $\left|\left\langle\eta \mid \mathscr{R}_{\boldsymbol{q}} \nabla H_{j} \phi_{j}\right\rangle\right| \leqslant \mathfrak{c}\|\eta\| /|\boldsymbol{q}| \leqslant \mathfrak{c}^{\prime}\|\eta\|$ on the support of $g$ by (B.7), and $\left|\boldsymbol{I}\left(\boldsymbol{q}, \mathscr{R}_{\boldsymbol{q}} \eta\right)\right| \leqslant \mathfrak{c}|\boldsymbol{q}|\left\|\mathscr{R}_{\boldsymbol{q}}\right\|\|\eta\| \leqslant \mathfrak{c}\|\eta\|$. Hence, $\boldsymbol{q} \mapsto g(\boldsymbol{q})\left\langle\eta \mid \mathscr{R}_{\boldsymbol{q}} \nabla H_{j} \phi_{j}\right\rangle$ and $\boldsymbol{q} \mapsto g(\boldsymbol{q}) \boldsymbol{I}\left(\boldsymbol{q}, \mathscr{R}_{\boldsymbol{q}} \eta\right)$ belong to $L^{2}\left[\mathbb{R}^{3}, \mathbb{C}^{3}\right]$. Since also $\left\langle\tilde{f}_{\boldsymbol{q}, \varepsilon} \mid \boldsymbol{G}\right\rangle=\left(h_{\varepsilon} * \boldsymbol{G}\right)(\boldsymbol{q}, \mu) \delta_{\lambda, \mu}$ and $h_{\varepsilon} * \boldsymbol{G} \rightarrow \boldsymbol{G}(\cdot, \mu) \delta_{\lambda, \mu}$ in $L^{2}\left[\mathbb{R}^{3}, \mathbb{C}^{3}\right]$ we conclude that

$$
\lim _{\varepsilon \searrow 0} C_{1}(\varepsilon)=-\mathfrak{e} \int_{\mathbb{R}^{3}} g(\boldsymbol{q}) \boldsymbol{G}_{j}(\boldsymbol{q}, \mu) \cdot\left\langle\eta \mid \mathscr{R}_{\boldsymbol{q}} \nabla H_{j} \phi_{j}\right\rangle \mathrm{d}^{3} \boldsymbol{q},
$$




$$
\begin{aligned}
\lim _{\varepsilon \searrow 0} C_{4}(\varepsilon)= & \mathfrak{e} \int_{\mathbb{R}} \int_{\mathbb{R}^{3}} g(\boldsymbol{k})\left\langle R(i y)\left(\boldsymbol{\alpha} \cdot \boldsymbol{G}_{j}(\boldsymbol{k}, \mu)\right) R(i y)(\boldsymbol{\alpha} \cdot \boldsymbol{k}) R_{\boldsymbol{k}}(i y)\right. \\
& \times \mathscr{R}_{\boldsymbol{k}} \eta\left|\phi_{j}\right\rangle \mathrm{d}^{3} \boldsymbol{k} \frac{y \mathrm{~d} y}{i \pi} .
\end{aligned}
$$

Next, we show that $C_{5}(\varepsilon)$ goes to zero: we have $\operatorname{supp}(g) \subset\{r \leqslant|\boldsymbol{q}| \leqslant 1 / r\}$, for some $r>0$, and we shall always assume that $0<\varepsilon \leqslant r / 2$. Then $\boldsymbol{q} \in \operatorname{supp}(g)$ and $h_{\varepsilon}(\boldsymbol{k}-\boldsymbol{q}) \neq 0$ implies $1 /|\boldsymbol{q}| \leqslant 1 / r$ and $1 \leqslant 2|\boldsymbol{k}| / r$. By Fubini's theorem we thus have

$$
\begin{aligned}
C_{5}(\varepsilon) \leqslant & \int_{\mathbb{R}} \int_{\mathbb{R}^{3}} \int_{\mathbb{R}^{3}}|g(\boldsymbol{q})| h_{\varepsilon}(\boldsymbol{k}-\boldsymbol{q})|\boldsymbol{k}-\boldsymbol{q}| \mid\left\langle\boldsymbol{\alpha}\left\{D_{\boldsymbol{q}} R_{\boldsymbol{q}}(i y) \mathscr{R}_{\boldsymbol{q}}\right\} \eta\right| \\
& \left.\times a(\boldsymbol{k}, \mu) \Theta^{-1 / 2} F(y) \Theta^{1 / 2} \phi_{j}\right\rangle \mid \mathrm{d}^{3} \boldsymbol{k} \mathrm{d}^{3} \boldsymbol{q} \frac{\mathrm{d} y}{\pi} .
\end{aligned}
$$

In the previous expression we have $|\boldsymbol{k}-\boldsymbol{q}| \leqslant \varepsilon$ if $h_{\varepsilon}(\boldsymbol{k}-\boldsymbol{q}) \neq 0$. Furthermore,

$$
\|\{\cdots\}\| \leqslant\left\|D_{\boldsymbol{q}} R_{\boldsymbol{q}}(i y)\left|D_{\boldsymbol{q}}\right|^{-1 / 2}\right\|\left\|\left|D_{\boldsymbol{q}}\right|^{1 / 2} \mathscr{R}_{\boldsymbol{q}}\right\| \leqslant \mathfrak{c}\left(1+y^{2}\right)^{-1 / 4} / r .
$$

We estimate the remaining factors of the integrand by Young's inequality, $|\langle u \mid v * w\rangle| \leqslant \mathfrak{c}\|u\|_{2}\|v\|_{1}\|w\|_{2}$, applied to the $\mathrm{d}^{3} \boldsymbol{k} \mathrm{d}^{3} \boldsymbol{q}$-integration. In this way we obtain

$$
\begin{aligned}
\left|C_{5}(\varepsilon)\right| \leqslant & \frac{\mathfrak{c} \varepsilon}{r}\left\|h_{\varepsilon}\right\|_{1}\|g\|_{2}\|\eta\| \\
& \cdot \int_{\mathbb{R}}\left\{(2 / r) \int_{|\boldsymbol{k}| \geqslant r / 2}|\boldsymbol{k}|\left\|a(\boldsymbol{k}, \mu) \Theta^{-1 / 2} F(y) \Theta^{1 / 2} \phi_{j}\right\|^{2} \mathrm{~d}^{3} \boldsymbol{k}\right\}^{1 / 2} \frac{\mathrm{d} y}{\left(1+y^{2}\right)^{1 / 4}} .
\end{aligned}
$$

Here, the integral $\int_{|\boldsymbol{k}| \geqslant r / 2} \ldots d^{3} \boldsymbol{k}$ is not greater than

$$
\left\|\left(H_{\mathrm{f}}^{(j)}\right)^{1 / 2} \Theta^{-1 / 2} F(y) \Theta^{1 / 2} \phi_{j}\right\|^{2} \leqslant\|F(y)\|^{2}\left\|H_{j}^{1 / 2} \phi_{j}\right\|^{2} \leqslant \mathfrak{c}\left(1+y^{2}\right)^{-1} .
$$

Since also $\left\|h_{\varepsilon}\right\|_{1}=1$ we conclude that $C_{5}(\varepsilon) \rightarrow 0$, as $\varepsilon \searrow 0$.

Obviously, $C_{2}$ and $C_{3}$ can also be treated by means of Young's inequality and we easily verify that $\lim _{\varepsilon \searrow 0} C_{2}(\varepsilon)=\lim _{\varepsilon \searrow 0} C_{3}(\varepsilon)=0$, again using that || $\boldsymbol{q}|-| \boldsymbol{k}|| \leqslant|\boldsymbol{k}-\boldsymbol{q}| \leqslant \varepsilon$, when $h_{\varepsilon}(\boldsymbol{k}-\boldsymbol{q}) \neq 0$. For $C_{3}$ we actually find

$$
C_{3}(\varepsilon) \leqslant \mathfrak{c}(\varepsilon / r)\|g\|_{2}\left\|h_{\varepsilon}\right\|_{1}\|\eta\|(2 / r)^{1 / 2}\left\|\left(H_{\mathrm{f}}^{(j)}\right)^{1 / 2} S \phi_{j}\right\|, \quad 0<\varepsilon \leqslant r / 2,
$$

where last norm $\left\|\left(H_{\mathrm{f}}^{(j)}\right)^{1 / 2} S \phi_{j}\right\|$ is well defined and bounded because of (A.3) and $\phi_{j} \in \operatorname{dom}\left(H_{\mathrm{f}}^{(j)}\right)$.

Putting everything together we see that $\left\langle\eta \mid a\left(\bar{g}_{\mu}\right) \phi_{j}\right\rangle$ is equal to the sum of terms on the RHS of (D.5) and (D.6). As this holds true, for every $g_{\mu}(k)=g(\boldsymbol{k}) \cdot \delta_{\mu, \lambda}$ with $g \in C_{0}^{\infty}\left[\mathbb{R}^{3} \backslash\{0\}\right]$, we conclude that

$$
\begin{aligned}
\left\langle\eta \mid a(\boldsymbol{k}, \mu) \phi_{j}\right\rangle= & -\mathfrak{e} \boldsymbol{G}_{j}(\boldsymbol{k}, \mu) \cdot\left\langle\eta \mid \mathcal{R}_{\boldsymbol{k}}(|\boldsymbol{k}|) \nabla H_{j} \phi_{j}\right\rangle \\
& +\mathfrak{e} \int_{\mathbb{R}}\left\langle R(i y)\left(\boldsymbol{\alpha} \cdot \boldsymbol{G}_{j}(\boldsymbol{k}, \mu)\right) R(i y)(\boldsymbol{\alpha} \cdot \boldsymbol{k}) R_{\boldsymbol{k}}(i y) \mathscr{R}_{\boldsymbol{k}} \eta \mid \phi_{j}\right\rangle \frac{y \mathrm{~d} y}{i \pi},
\end{aligned}
$$


for all $\boldsymbol{k} \in \mathbb{R}^{3} \backslash N_{\eta}$, where $N_{\eta}$ is some $\eta$-dependent zero set. Applying this results to all $\eta$ in some countable dense domain in $\mathscr{H}$ we obtain (6.1).

\section{References}

[1] Abdesselam, A., Hasler, D.: Analyticity of the ground state energy for massless Nelson models. Comm. Math. Phys. 310, 511-536 (2012)

[2] Bach, V., Chen, T., Faupin, J., Fröhlich, J., Sigal, I.M.: Effective dynamics of an electron coupled to an external potential in non-relativistic QED. Ann. Henri Poincaré arXiv:1202.3189 (2012). doi:10.1007/s00023-012-0222-8

[3] Bach, V., Chen, T., Fröhlich, J., Sigal, I.M.: The renormalized electron mass in non-relativistic quantum electrodynamics. J. Funct. Anal. 243, 426-535 (2007)

[4] Bach, V., Fröhlich, J., Pizzo, A.: Infrared-finite algorithms in QED: the groundstate of an atom interacting with the quantized radiation field. Commun. Math. Phys. 264, 145-165 (2006)

[5] Bach, V., Fröhlich, J., Pizzo, A.: An infrared-finite algorithm for Rayleigh scattering amplitudes, and Bohr's frequency condition. Commun. Math. Phys. 274, 457-486 (2007)

[6] Bach, V., Fröhlich, J., Pizzo, A.: Infrared-finite algorithms in QED. II. The expansion of the groundstate of an atom interacting with the quantized radiation field. Adv. Math. 220, 1023-1074 (2009)

[7] Bach, V., Könenberg, M.: Construction of the ground state in nonrelativistic QED by continuous flows. J. Differ. Equs. 231, 693-713 (2006)

[8] Bachmann, S., Deckert, D.A., Pizzo, A.: The mass shell of the Nelson model without cut-offs. J. Funct. Anal. 263, 1224-1282 (2012)

[9] Chen, T.: Infrared renormalization in non-relativistic QED and scaling criticality. J. Funct. Anal. 254, 2555-2647 (2008)

[10] Chen, T., Fröhlich, J.: Coherent infrared representations in non-relativistic QED. In: Spectral theory and mathematical physics: a Festschrift in honor of Barry Simon's 60th birthday. (Proceedings of the Symposium on Pure Mathematics), vol. 76, pp. 25-45. Am. Math. Soc., Providence (2007)

[11] Chen, T., Fröhlich, J., Pizzo, A.: Infraparticle scattering states in non-relativistic quantum electrodynamics. II. Mass shell properties. J. Math. Phys. 50(012103), 34 (2009)

[12] Chen, T., Fröhlich, J., Pizzo, A.: Infraparticle scattering states in non-relativistic QED. I. The Bloch-Nordsieck paradigm. Commun. Math. Phys. 294, 761-825 (2010)

[13] Deckert, D.A., Pizzo, A.: Ultraviolet properties of the spinless, one-particle Yukawa Model. Preprint, arXiv:1208.2646 (2012)

[14] Fröhlich, J.: On the infrared problem in a model of scalar electrons and massless, scalar bosons. Ann. Inst. Henri Poincaré Sect. A (N.S.) 19, 1-103 (1973)

[15] Fröhlich, J.: Existence of dressed one electron states in a class of persistent models. Fortschritte Phys. 22, 159-198 (1974)

[16] Fröhlich, J., Griesemer, M., Schlein, B.: Asymptotic electromagnetic fields in models of quantum-mechanical matter interacting with the quantized radiation field. Adv. Math. 164, 349-398 (2001) 
[17] Fröhlich, J., Pizzo, A.: Renormalized electron mass in nonrelativistic QED. Commun. Math. Phys. 294, 439-470 (2010)

[18] Hasler, D., Herbst, I.: Absence of ground states for a class of translation invariant models of non-relativistic QED. Commun. Math. Phys. 279, 769-787 (2008)

[19] Hiroshima, F., Sasaki, I.: On the ionization energy of the semi-relativistic PauliFierz model for a single particle. RIMS Kokyuroku Bessatsu. 21, 25-34 (2010)

[20] Hiroshima, F., Spohn, H.: Ground state degeneracy of the Pauli-Fierz Hamiltonian with spin. Adv. Theor. Math. Phys. 5, 1091-1104 (2001)

[21] Kato, T.: Perturbation theory for linear operators, Classics in Mathematics. Springer, Berlin (1995)

[22] Könenberg, M., Matte, O.: Ground states of semi-relativistic Pauli-Fierz and no-pair Hamiltonians in QED at critical Coulomb coupling. J. Operator Theory 70(1) (2013)

[23] Könenberg, M., Matte, O.: On enhanced binding and related effects in the nonand semi-relativistic Pauli-Fierz models. arXiv:1207.5638 to appear in Commun. Math. Phys. (2012)

[24] Könenberg, M., Matte, O., Stockmeyer, E.: Existence of ground states of hydrogen-like atoms in relativistic quantum electrodynamics I: The semirelativistic Pauli-Fierz operator. Rev. Math. Phys. 23, 375-407 (2011)

[25] Könenberg, M., Matte, O., Stockmeyer, E.: Existence of ground states of hydrogen-like atoms in relativistic quantum electrodynamics II: The no-pair operator. J. Math. Phys. 52, 123501 (2011)

[26] Matte, O.: On higher order estimates in quantum electrodynamics. Documenta Math. 15, 207-234 (2010)

[27] Matte, O., Stockmeyer, E.: Exponential localization of hydrogen-like atoms in relativistic quantum electrodynamics. Commun. Math. Phys. 295, 551-583 (2010)

[28] Miyao, T., Spohn, H.: Spectral analysis of the semi-relativistic Pauli-Fierz Hamiltonian. J. Funct. Anal. 256, 2123-2156 (2009)

[29] Møller, J.S.: The translation invariant massive Nelson model. I. The bottom of the spectrum. Ann. Henri Poincaré. 6, 1091-1135 (2005)

[30] Pizzo, A.: One-particle (improper) states in Nelson's massless model. Ann. Henri Poincaré. 4, 439-486 (2003)

[31] Pizzo, A.: Scattering of an Infraparticle: the one particle sector in Nelson's massless model. Ann. Henri Poincaré. 6, 553-606 (2005)

[32] Reed, M., Simon, B.: Methods of modern mathematical physics. II. Fourier analysis, self-adjointness. Academic Press, New York (1975)

[33] Schroer, B.: Infrateilchen in der Quantenfeldtheorie. Fortschritte Phys. 11, $1-32(1963)$

Martin Könenberg

Fakultät für Physik

Universität Wien

Boltzmanngasse 5

1090 Vienna

Austria

e-mail: martin.koenenberg@univie.ac.at 
Oliver Matte

Mathematisches Institut

Ludwig-Maximilians-Universität

Theresienstraße 39

80333 München, Germany

Present address:

Oliver Matte

Institut for Matematik

Århus Universitet

Ny Munkegade 118

8000 Århus C, Denmark

e-mail: matte@math. lmu.de

Communicated by Jan Derezinski.

Received: April 23, 2012.

Accepted: April 26, 2013. 